\title{
Beyond the Standard Models with Cosmic Strings
}

\author{
Yann Gouttenoire ${ }^{a}$, Géraldine Servant ${ }^{a, b}$, Peera Simakachorn ${ }^{a}$ \\ a DESY, Notkestraße 85, D-22607 Hamburg, Germany \\ ${ }^{b}$ II. Institute of Theoretical Physics, Universität Hamburg, 22761 Hamburg, Germany
}

\begin{abstract}
We examine which information on the early cosmological history can be extracted from the potential measurement by third-generation gravitational-wave observatories of a stochastic gravitational wave background (SGWB) produced by cosmic strings. We consider a variety of cosmological scenarios breaking the scale-invariant properties of the spectrum, such as early long matter or kination eras, short intermediate matter and inflation periods inside a radiation era, and their specific signatures on the SGWB. This requires to go beyond the usually-assumed scaling regime, to take into account the transient effects during the change of equation of state of the universe. We compute the time evolution of the string network parameters and thus the loop-production efficiency during the transient regime, and derive the corresponding shift in the turning-point frequency. We consider the impact of particle production on the gravitational-wave emission by loops. We estimate the reach of future interferometers LISA, BBO, DECIGO, ET and $\mathrm{CE}$ and radio telescope SKA to probe the new physics energy scale at which the universe has experienced changes in its expansion history. We find that a given interferometer may be sensitive to very different energy scales, depending on the nature and duration of the non-standard era, and the value of the string tension. It is fascinating that by exploiting the data from different GW observatories associated with distinct frequency bands, we may be able to reconstruct the full spectrum and therefore extract the values of fundamental physics parameters.
\end{abstract}




\section{Contents}

1 Introduction 4

2 Recap on Cosmic Strings 6

2.1 String field theory . . . . . . . . . . . . . . . . . . 6

2.2 Cosmic-string network formation and evolution . . . . . . . . . . 7

2.3 Decay channels of Cosmic Strings . . . . . . . . . . . . . . . 8

2.4 Constraints on the string tension $G \mu$ from GW emission $\ldots \ldots$. . . . 10

3 Gravitational waves from cosmic strings 11

3.1 Beyond the Nambu-Goto approximation . . . . . . . . . . . . . . 11

3.2 Assumptions on the loop distribution . . . . . . . . . . . . . . . 13

3.3 The gravitational-wave spectrum . . . . . . . . . . . . . . . 15

3.4 The frequency - temperature relation . . . . . . . . . . . 17

3.5 The astrophysical foreground . . . . . . . . . . . . . . 18

4 The Velocity-dependent One-Scale model 19

4.1 The loop-production efficiency . . . . . . . . . . . . . . . . . . . . 19

4.2 The VOS equations . . . . . . . . . . . . . . . . . . . . 19

4.3 Scaling regime solution and beyond . . . . . . . . . . . 20

5 Standard cosmology 21

5.1 The cosmic expansion . . . . . . . . . . . . . . . . . . 21

5.2 Gravitational wave spectrum . . . . . . . . . . . . . . . 22

5.3 Deviation from the scaling regime . . . . . . . . . . . . . . . 22

5.4 Beyond the NG approximation . . . . . . . . . . . . . . . . . . . 22

5.5 Initial network configuration . . . . . . . . . . . . . . . 22

6 Long-lasting matter or kination era 25

6.1 The non-standard scenario . . . . . . . . . . . . . . 25

6.2 Impact on the spectrum: a turning-point . . . . . . . . . . . 25

6.3 Constraints . . . . . . . . . . . . . . . . 27

6.4 A shorter period of kination . . . . . . . . . . . . . 27

7 Intermediate matter era $\quad 30$

7.1 The non-standard scenario . . . . . . . . . . . . . . . . 30

7.2 Impact on the spectrum: a low-pass filter . . . . . . . . . . . . . 30

7.3 Constraints . . . . . . . . . . . . . . . . . 31

8 Intermediate inflationary era $\quad 32$

8.1 The non-standard scenario . . . . . . . . . . . . . . . . . 32

8.2 The stretching regime and its impact on the spectrum . . . . . . . . 33

8.3 Constraints . . . . . . . . . . . . . . . . 37 
9 Detectability of spectral features $\quad 38$

9.1 Two prescriptions . . . . . . . . . . . . . . . . . . . 38

9.2 More details on the turning-point prescription . . . . . . . . . . . . . 40

9.3 Comparative reach of different observatories at a glance . . . . . . . . . . 42

10 Summary and conclusion $\quad 42$

A Cosmic string detection beyond GW $\quad 45$

A.1 Gravitational lensing . . . . . . . . . . . . . . . . 45

A.2 Temperature anisotropies in the CMB . . . . . . . . . . . . 45

B Derivation of the GW spectrum from CS 45

B.1 From GW emission to detection . . . . . . . . . . . . . . 46

B.2 From loop production to GW emission . . . . . . . . . . . . 46

B.3 The loop production . . . . . . . . . . . . . . . . . . 47

B.4 The master equation . . . . . . . . . . . . . . 47

C Derivation of the frequency - temperature relation 47

C.1 In standard cosmology . . . . . . . . . . . . . . . . . 47

C.2 During a change of cosmology . . . . . . . . . . . . . . . . . 49

C.3 In the presence of an intermediate inflation period . . . . . . . . . . 49

C.4 Cut-off from particle production . . . . . . . . . . . . . . . 49

D Derivation of the VOS equations $\quad 49$

D.1 The NG string in an expanding universe . . . . . . . . . . . . . . 49

D.2 The long-string network . . . . . . . . . . . . . . 50

D.3 VOS 1: the correlation length . . . . . . . . . . . . 50

D.4 Thermal friction . . . . . . . . . . . . . . . . . 51

D.5 VOS 2: the mean velocity ...................... 51

E Extension of the original VOS model 52

E.1 VOS model from NG simulations . . . . . . . . . . . . . . . . 52

E.2 VOS model from AH simulations . . . . . . . . . . . . . . 52

E.3 VOS model from AH simulations with particle production . . . . . . . 52

F Sensitivity curves of GW detectors 53

F.1 The signal-to-noise ratio . . . . . . . . . . . . . . . 53

F.2 The power-law integrated sensitivity curve . . . . . . . . . . . 54

F.3 Results . . . . . . . . . . . . . . . . . . . . . 54 


\section{Introduction}

The Standard Model of particle physics needs to be completed to address observational facts such as the matter antimatter asymmetry and the dark matter of the universe, as well as the origin of inflation. These, together with a number of other fundamental theoretical puzzles associated with e.g. the flavour structure of the matter sector and the ultra-violet properties of the Higgs scalar field, motivate extensions of the Standard Model featuring new degrees of freedom and new energy scales. In turn, such new physics can substantially impact the expansion history in the early universe and leads to deviations with respect to the standard cosmological model. Any deviations in the Friedmann equation occurring at temperatures above the $\mathrm{MeV}$ remain to date essentially unconstrained.

In the standard cosmological model, primordial inflation is followed by a long period of radiation domination until the more recent transitions to matter and then dark energy domination. Evidence for this picture comes primarily from observations of the Cosmic Microwave Background (CMB) and the successful predictions of Big-Bang Nucleosynthesis $(\mathrm{BBN})$, which on the other hand, do not allow to test cosmic temperatures above $\mathcal{O}(\mathrm{MeV})$.

An exciting prospect for deciphering the pre-BBN universe history and therefore high energy physics unaccessible by particle physics experiments, comes from the possible detection of a stochastic background of gravitational waves (SGWB), originating either from cosmological phase transitions, from cosmic strings or from inflation [1].

Particularly interesting are cosmic strings (CS), which act as a long-lasting source of gravitational waves $(\mathrm{GW})$ from the time of their production, presumably very early on, until today. The resulting frequency spectrum therefore encodes information from the almost entire cosmic history of our universe, and could possibly reveal precious details about the high energy particle physics responsible for a modified universe expansion.

There has been a large literature on probes of a non-standard cosmology through the nearly-scale invariant primordial GW spectrum generated during inflation [2-20]. In contrast, little efforts have been invested to use the scale-invariant GW spectrum generated by CS [21-25] while there has been intense activity on working out predictions for the SGWB produced by CS in standard cosmology [26-42].

In this paper, we propose to use the detection of a SGWB from local cosmic strings to test the existence of alternative stages of cosmological expansion between the end of inflation and the end of the radiation era. Particularly well-motivated is a stage of early-matter domination era induced by a heavy cold particle dominating the universe and decaying before BBN. Another possibility is a stage of kination triggered by the fast rolling evolution of a scalar field down its potential. Finally, supercooled confining phase transitions [43-50] can induce some late short stages of inflation inside a radiation era. The latter were motivated at the $\mathrm{TeV}$ scale but the properties of the class of scalar potentials naturally inducing a short inflationary era can be applied to any other scale. We will consider these various possibilities and their imprints on the GW spectrum from cosmic strings.

The dominant source of GW emission from a cosmic string network comes from loops which are continuously formed during the network fragmentation. We thus primarily need to compute the loop-production efficiency during the non-standard transition eras. This is crucial for a precise prediction of the turning-point frequency as a signature of the non-standard era. The temperature of the universe at the end of the non-standard 
era can be deduced from the measurement of these turning point frequencies.

The observational prospects for measuring the SGWB from cosmic string networks at LISA was recently reviewed in [23]. Besides, the effect of particle production on the loop distribution and thus on the SGWB was recently discussed [51, 52] where it was however concluded that the expected cutoff is outside the range of current and planned detectors. Our paper integrates these recent developments and goes beyond in several directions:

- We go beyond the so-called scaling regime by computing the time evolution of the string network parameters (long string mean velocity and correlation length) and thus the loop-production efficiency during modifications of the equation of state of the universe. Including these transient effects results in a turning-point frequency smaller by $\mathcal{O}(20)$ compared to the prediction from the scaling regime ${ }^{1}$.

- As a result, the energy scale of the universe associated with the departure from the standard radiation era that can be probed is correspondingly larger than the one predicted from scaling networks.

- We investigate a large variety of non-standard cosmologies, in particular models where a non-standard era can be rather short inside the radiation era, due for instance to some cold particle temporarily dominating the energy density (short matter era) [53] or some very short stage of inflation (for a couple of efolds) due to a high-scale supercooled confinement phase transition. Such inflationary stages occurring at scales up to $10^{14} \mathrm{GeV}$ could be probed. Even 1 or 2 e-folds could lead to observable features.

- We also consider longer low-scale inflation models. For instance, an intermediate inflationary era lasting for $\mathcal{O}(10)$ efolds, the SGWB from cosmic strings completely looses its scale invariant shape and has a peak structure instead. A TeV scale inflation era can lead to a broad peak either in the LISA or BBO band or even close to the SKA band, depending on the precise value of the string tensions $G \mu$, and the number of efolds $N_{e}$.

- We include cutoff effects from particle production which can limit observations for small value of the string tension $G \mu \lesssim 10^{-15}$.

- We provide the relations between the observed frequency of a given spectral feature and the energy scale of the universe for different physical effects: i) the end of a non-standard matter or kination era; ii) the time when particle emission starts to dominate; iii) the time at which the CS network re-enters the horizon after an intermediate inflation era.

The plan of the paper is the following. In Section 2, we recap the key features of CS networks, their cosmological evolution, decay channels and the pulsar timing array constraints on the string tension. Section 3 reviews the computation of the SGWB from Nambu-Goto CS. We first discuss the underlying assumptions on the small-scale structure and on the loop distribution and then derive the master formula of the GW frequency spectrum. An important discussion concerns the non-trivial frequency-temperature relation and how it depends on the cosmological scenario. Sec. 4 is devoted to the derivation

1 The turning-point frequency can even be smaller by $\mathcal{O}(400)$ if in a far-future, a precision of the order of $1 \%$ can be reached in the measurement of the SGWB. 
of the loop production efficiency beyond the scaling regime, taking into account transient effects from the change in the the equation of state of the universe. We apply this to predict the SGWB in the standard cosmological model in Sec. 5. We then move to discuss non-standard cosmological histories, a long-lasting matter or kinetion era before the radiation era in Sec. 6, a short intermediate matter era inside the radiation era in Sec. 7, and an intermediate inflationary era in Sec. 8. We discuss the specific spectral features in each of these cases and their observability by future instruments. Section 9 summarises proposed approaches to test different scenarios and the physics reach of each experiment. We conclude in Sec. 10. Additional details are moved to appendices, such as non-GW constraints on the string tension $G \mu$ in App. A, a step-by-step derivation of the GW spectrum in App. B, the formulae of the various turning-point frequencies in App. C, the derivation of the VOS equations in App. D, a discussion of the extensions to the original VOS model in App. E and the calculation of the integrated power-law sensitivity curves for each experiment in App. F

\section{Recap on Cosmic Strings}

Cosmic strings have been the subject of numerous studies since the pioneering paper [54], see [55-57] for reviews.

\subsection{String field theory}

A topological defect: CS can originate as fundamental or composite objects in string theory [58-65] or as topological defects from spontaneous symmetry breaking (SSB) when the vacuum manifold $\mathcal{M}$ has a non-trivial first homotopy group $\pi_{1}(\mathcal{M})$. Any theory with spontaneous breaking of a $U(1)$ symmetry has a string solution, since $\pi_{1}(U(1))=\mathbb{Z}$. More complex vacuum manifolds with string solutions can appear in various grand unified theories [66-69], e.g. $S O(10) \rightarrow S O(5) \times \mathbb{Z}_{2}$.

The abelian-Higgs model: The standard example of field theories with a string-liked solution is the Abelian-Higgs (AH) model, a field theory with a complex scalar field $\phi$ charged under a $U(1)$ gauge interaction. Note that the symmetry can also be global. The resulting strings solutions corresponding to local and global symmetries are called local and global strings, respectively. CS correspond to lines where the scalar field sits on the top of its mexican hat potential $V(\phi)$ and approaches its vacuum expectation value (VEV) at large distance, the Nielsen-Olesen vortex [70]. When following a closed path around the string, the phase of the complex scalar field returns to its original value after winding around the mexican hat an integer $n$ number of times. The energy per unit of length, also known as the string tension reads [56]

$$
\mu \approx 2 \pi \eta^{2} n \times \begin{cases}1 & \text { for local strings, } \\ \ln \left(\frac{m_{\phi}}{H}\right) & \text { for global strings, }\end{cases}
$$

with $\eta$ the scalar field VEV. The Hubble horizon $H^{-1}$ and the string core width $m_{\phi}^{-1}$ play the role of IR and UV cut-offs. The logarithmic divergence of the tension of global strings is due to the existence of a long-range interaction mediated by the massless Goldstone mode (the complex phase of $\phi$ ). 


\subsection{Cosmic-string network formation and evolution}

Kibble mechanism: The formation of cosmic strings occurs during a cosmological phase transition associated with spontaneous symmetry breaking, occurring at a temperature, approximately given by the VEV acquired by the scalar field

$$
T_{\mathrm{p}} \sim 10^{11} \mathrm{GeV}\left(\frac{G \mu}{10^{-15}}\right)^{1 / 2} .
$$

CS are randomly distributed and form a network characterized by its correlation length $L$, which can be defined as

$$
L \equiv \sqrt{\mu / \rho_{\infty}}
$$

where $\mu$ is the string tension, the energy per unit length, and $\rho_{\infty}$ is the energy density of long strings. More precisely, long strings form infinite random walks [71] which can be visualized as collections of segments of length $L$.

Loop chopping: Each time two segments of a long string cross each other, they intercommute, with a probability $P$ and form a loop. Loop formation is the main energyloss mechanism of the long string network. In numerical simulations [72] and analytical modelling [73], the probability of inter-commutation has been found to be $P=1$ but in some models it can be lower. This is the case of models with extra-dimensions [59, 74], strings with junctions [75] or peeling [76], or the case of highly relativistic strings [77].

Scaling regime: Just after the network is formed, the strings may interact strongly with the thermal plasma such that their motion is damped. When the damping stops, cosmic strings oscillate and enter the phase of scaling evolution. During this phase, the network experiences two competing dynamics:

1. Hubble stretching: the correlation length scale stretches due to the cosmic expansion, $L \sim a$.

2. Fragmentation of long strings into loops: a loop is formed after each segment crossing. Right after their formation, loops evolve independently of the network and start to decay through gravitational radiation and/or particle production.

It is known since a long time ago [78-82], that out of the two competing dynamics, Hubble expansion and loop fragmentation, there is an attractor solution, called the scaling regime, where the correlation length scales as the cosmic time,

$$
L \sim t
$$

Note however that in the case of global-string network, it has been claimed that the scaling property in eq. (4), is logarithmically violated due to the dependence of the string tension on the Hubble horizon [83-88]. More recently, an opposite conclusion has been drawn in [89]. 
Number of strings: During the scaling regime, the number of strings per Hubble patch is conserved

$$
\frac{\rho_{\infty} H^{-3}}{\mu L}=\text { constant }
$$

Moreover, the energy density of the long-string network, which scales as $\rho_{\infty} \sim \mu / t^{2}$, has the same equation-of-state as the main background cosmological fluid $\rho_{\mathrm{bkg}} \sim a^{-n}$,

$$
\frac{\rho_{\infty}}{\rho_{\mathrm{bkg}}} \sim \frac{a^{n}}{t^{2}} \sim \text { constant }
$$

where we used $a=t^{2 / n}$. Hence, the long-string energy density redshifts as matter during matter domination and as radiation during radiation domination. The scaling regime allows cosmic strings not to dominate the energy density of the universe, unlike other topological defects. The scaling property of a string network has been checked some fifteen years ago in numerical Nambu-Goto simulations [90-93] and more recently with larger simulations [94]. During the scaling regime, the loop production function is scalefree, with a power-law shape, meaning that loops are produced at any size between the Hubble horizon $t$ and the scale $\sim \Gamma G \mu t$, below which the strings have been smoothened by the gravitational backreaction and there is no further segment crossing.

A scale-invariant SGWB: An essential outcome is the scale-invariance of the Stochastic GW Background generated by loops during the scaling regime [26-42]. Remarkably, the spectrum generated by loops produced during radiation domination is flat, $\propto f^{0}$, and an early matter domination or an early kination-domination era turns the spectral index from $f^{0}$ to respectively $f^{-1}$ or $f^{1}[21,22]$. We give more details in sec. 3.3. Hence, the detection of the SGWB from CS by LIGO [95], DECIGO, BBO [96], LISA [97], Einstein Telescope [98, 99] or Cosmic Explorer [100] would offer an unique observation window on the equation of state of the Universe at the time when the CS loops responsible for the detected GW are formed. In sec. 6, 7 and 8, we study the possibility for probing particular non-standard cosmological scenario: long matter/kination era, intermediate matter and intermediate inflation, respectively.

\subsection{Decay channels of Cosmic Strings}

Cosmic strings can decay in several ways, as we discuss below.

GW radiation from long strings: Because of their topological nature, straight infinitely-long strings are stable against decay. However, small-scale structures of wiggly long strings can generate gravitational radiation. Intuitively, a highly wiggly string can act as a gas of small loops. The GW emission from long strings can be neglected compared to the GW emission from loops, as loops live much longer than a Hubble time $[32,56]$. Indeed, the GW signal emitted by loops is enhanced by the large number of loops (continuously produced). Nambu-Goto numerical simulations have shown that the loop energy density is at least 100 times larger than the long-string energy density [101]. Only for global strings where loops are short-lived due to efficient Goldstone production, the GW emission from long strings may be the dominant SGWB [102-105]. In what follows, we only consider the emission from loops. 


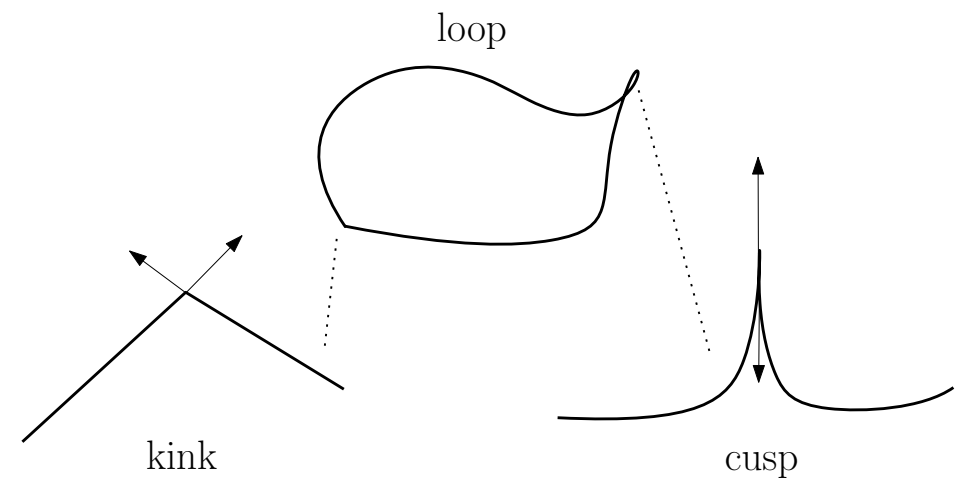

Figure 1: Cartoon showing the geometry of a kink and a cusp which are singular structures formed on loops. The arrows denote the tangent vectors of the string segments.

GW radiation from loops (local strings): In contrast to long strings, loops do not contain any topological charge and are free to decay into GW. The GW radiation power is found to be [56]

$$
P_{\mathrm{GW}}=\Gamma G \mu^{2}
$$

where the total GW emission efficiency $\Gamma$ is determined from NG simulations, $\Gamma \simeq 50$ [106]. Note that the gravitational power radiated by a loop is independent of its length. This can be understood from the quadrupole formula $P=G / 5\left(Q^{\prime \prime \prime}\right)^{2}[28,107]$ where the triple time derivative of the quadrupole, $Q^{\prime \prime \prime} \propto$ mass $(\text { length })^{2} /(\text { time })^{3} \propto \mu$, is indeed independent of the length. The resulting GW are emitted at frequencies [55, 108]

$$
f=\frac{2 k}{l}, \quad k \in \mathbb{Z}^{+},
$$

corresponding to the proper modes $k$ of the loop. The frequency dependence of the power spectrum $P_{\mathrm{GW}}(k)$ relies on the nature of the loop small-scale structures [109, 110], e.g. kinks or cusps, c.f. fig. 1. More precisely, the spectrum of the gravitational power emitted from one loop reads

$$
P_{\mathrm{GW}}^{(k)}=\Gamma^{(k)} G \mu^{2}, \quad \text { with } \quad \Gamma^{(k)} \equiv \frac{\Gamma k^{-n}}{\sum_{p=1}^{\infty} p^{-n}}, \quad n= \begin{cases}4 / 3 & \text { cusps } \\ 5 / 3 & \text { kinks } \\ 2 & \text { kink-kink collisions }\end{cases}
$$

where the spectral index $n=4 / 3$ when the small-scale structure is dominated by cusps [28, 36, 111], $n=5 / 3$ for kink domination [36], or $n=2$ for kink-kink collision domination $[109,110]$. Therefore, immediately after a loop gets created, at time $t_{i}$ with a length $\alpha t_{i}$, its length $l(t)$ shrinks through emission of GW with a rate $\Gamma G \mu$

$$
l(t)=\alpha t_{i}-\Gamma G \mu\left(t-t_{i}\right) .
$$

Consequently, the string lifetime due to decay into GW is given by

$$
\tau_{\mathrm{GW}}=\frac{\alpha t_{i}}{\Gamma G \mu} .
$$

The superposition of the GW emitted from all the loops formed since the creation of the long-string network generates a Stochastic GW Background. Also, cusp formations can emit high-frequency, short-lasting GW bursts [35, 36, 109, 110, 112]. If the rate of such events is lower than their frequency, they might be subtracted from the SGWB. 
Goldstone boson radiation (global strings): For global strings, the massless Goldstone particle production is the main decay channel. The radiation power has been estimated [56]

$$
P_{\text {Gold }}=\Gamma_{\text {Gold }} \mu,
$$

where $\Gamma_{\text {Gold }} \approx 100$ [113]. We see that the GW emission power in eq. (7) is suppressed by a factor $G \mu$ with respect to the Goldstone emission power in eq. (12). Therefore, for global strings, the loops decay into Goldtone bosons after a few oscillations before having the time to emit GW [56]. However, recent studies claim that the SGWB from global string might be detectable [25, 114].

A well-motivated example of global string is the axion string coming from the breaking of a $U(1)$ Peccei-Quinn symmetry [113, 115-117]. Its phenomenological interest relies on the possibility to generate the correct Dark Matter abundance from Goldstone production. However, the value of the energy of the emitted Goldstone, needed to compute the axion relic abundance, is still matter of debate [84]. Particularly, the precise dependence of the emitted mean energy with the infrared cut-off $\mathrm{H}^{-1}$ is unknown and is not tractable in numerical simulations due to the too large hierarchy between the string core size and the Hubble horizon. Hence, in the case of post-inflationnary Peccei-Quinn breaking, it is currently not understood whether the axion abundance today coming from the decay of the axionic string network supersedes the abundance coming from the vacuum misalignement or not. Note, however [89] which claims that the dependence of the emitted energy with the cut-off is an artefact.

Massive particle radiation: When the string curvature size is larger than the string thickness, one expects the quantum field nature of the CS, like the possibility to radiate massive particles, to give negligible effects and one may instead consider the CS as an infinitely thin 1-dimensional classical object with tension $\mu$ : the Nambu-Goto (NG) string. However, due to the presence of small-scale structures on the strings, regions with curvature comparable to the string core size can develop and the NG approximation breaks down. In that case, massive radiation can be emitted during processes known as cusp annihilation [118] or kink-kink collisions [51]. We discuss massive particle emission in more details in sec. 3.1.

\subsection{Constraints on the string tension $G \mu$ from GW emission}

The observational signatures of Nambu-Goto cosmic strings are mainly gravitational. The GW emission can be probed by current and future pulsar timing arrays and GW interferometers, while the static gravitational field around the string can be probed by CMB, $21 \mathrm{~cm}$, and lensing observables, see app. A for more details on non-GW probes. The strongest constraints come from pulsar timing array EPTA, $G \mu \lesssim 8 \times 10^{-10}$ [119], and NANOGrav, $G \mu \lesssim 5.3 \times 10^{-11}$ [120]. Comparison with the theoretical predictions from the SGWB from cosmic strings leads to $G \mu \lesssim 2 \times 10^{-11}[22,106]$ or $G \mu \lesssim 10^{-10}$ [110], even though it can be relaxed to $G \mu \lesssim 5 \times 10^{-7}$ [38], after taking into account uncertainties on the loop size at formation and on the number of emitting modes. Note that it can also be strengthened by decreasing the inter-commutation probability [40, 121, 122].

By using the EPTA sensitivity curve derived in [123], we obtain the upper bound on $G \mu$, one order of magnitude higher, $2 \times 10^{-10}$, instead of $2 \times 10^{-11}$, cf. fig. 4 . This bound becomes $\sim 5 \times 10^{-11}$ by using the NANOGrav sensitivity curve derived in [123]. Another large source of uncertainty is the nature of the GW spectrum generated by a loop, which 
depends on the assumption on the loop small-scale structure (e.g. the number of cusps, kinks and kink-kink collisions per oscillations) [40, 110]. For instance, the EPTA bound can be strengthened to $G \mu \lesssim 6.7 \times 10^{-14}$ if the loops are very kinky [110]. CS can also emit highly-energetic and short-lasting GW bursts due to cusp formation [35, 36, 109, 110, 112]. From the non-observation of such events with LIGO/VIRGO [124, 125], one can constrain $G \mu \lesssim 4.2 \times 10^{-10}$ with the loop distribution function from [126]. However, the constraints are completely relaxed with the loop distribution function from [101].

\section{Gravitational waves from cosmic strings}

In this work, we do not consider the case of global strings where the presence of a massless Goldstone in the spectrum implies that particle production is the main energy loss so that GW emission is suppressed [56]. Recent proposals nevertheless claim a potential reach by next generation of GW interferometers [25, 114].

There has been a long debate in the community whether local cosmic strings mainly loose their energy via GW emission or by particle production. We summarise the arguments and clarify the underlying assumptions below.

\subsection{Beyond the Nambu-Goto approximation}

Quantum field string simulations: Quantum field string (Abelian-Higgs) lattice simulations run by Hindmarsh et al. [127-129] have shown that decay into massive radiation is the main energy loss and is sufficient to lead to scaling. Then, loops decay within one Hubble time into scalar and gauge boson radiation before having the time to emit GW. It is suggested that the presence of small-scale structures, kinks and cusps, at the string core size are responsible for the energy loss into particle production. In these regions of large string curvature, the NG approximation, which considers CS as infinitely thin 1-dimensional classical objects, is no longer valid.

However, Abelian-Higgs simulations run by [130-132] have claimed the opposite result, that energy loss into massive radiation is exponentially suppressed when the loop size is large compared to the thickness of the string.

Small-scale structure: At formation time, loops are not smooth but made of straight segments linked by kinks [133]. Kinks are also created in pairs after each string intercommutation, see [134] or fig. 2.1 in [135]. The presence of straight segments linked by kinks prevents the formation of cusps. However, backreaction from GW emission smoothens the shapes, hence allowing for the formation of cusps [133] (see fig. 1). Because of the large hierarchy between the gravitational backreaction scale and the cosmological scale $H$, the effects of the gravitational backreaction on the loop shape are not easily tractable numerically. The effects of backreaction from particle emission are shown in [134]. Nevertheless, it has been proposed since long [136] that the small-scale structures are smoothened below the gravitational backreaction scale $\sim \Gamma G \mu t$. Particularly, based on analytical modelling on simple loop models, it has been shown in $[137,138]$ that due to gravitational backreaction, kinks get rounded off, become closer to cusps and then cusps get weakened. In earlier works, the same authors $[139,140]$ claimed that whether the smoothening has the time to occur within the loop life time strongly depends on the initial loop shape. In particular, for a four-straight-segment loop, the farther from the square shape, the faster 
the smoothening, whereas for more general loop shapes, the smoothening may not always occur.

To summarise the last two paragraphs, the efficiency of the energy loss into massive radiation depends on the nature of the small-scale structure, which can be understood as a correction to the Nambu-Goto approximation. The precise nature of the smallscale structure, its connection with the gravitational backreaction scale and the conflict between Nambu-Goto and Abelian Higgs simulations remain to be explained. Moreover, the value of the gravitational backreaction scale itself, see sec. 3.2 is matter of debate. For our study, we follow the proposal of [52] for investigating how the GW spectrum is impacted for two benchmark scenarios: when the small-scale structures are dominated by cusps or when they are dominated by kinks. We give more details in the next paragraph.

Massive radiation emission: In the vicinity of a cusp, the topological charge vanishes where the string cores overlap. Hence, the corresponding portions of the string can decay into massive radiation. The length of the overlapping segment has been estimated to be $\sqrt{r l}[118,141]$ where $r \simeq \mu^{-1 / 2}$ is the string core size and $l$ is the loop length. Hence, the energy radiated per cusp formation is $\mu \sqrt{r l}$, from which we deduce the power emitted from a loop

$$
P_{\text {cusp }}^{\text {part }} \simeq N_{\mathrm{c}} \frac{\mu^{3 / 4}}{l^{1 / 2}}
$$

where $N_{\mathrm{c}}$ is the average number of cusps per oscillation, estimated to be $N_{\mathrm{c}} \sim 2$ [133]. Note that the consideration of pseudo-cusps, pieces of string moving at highly relativistic velocities, might also play a role $[142,143]$.

Even without the presence of cusps, Abelian-Higgs simulations [51] have shown that kink-kink collisions produce particles with a power per loop

$$
P_{\mathrm{kink}}^{\mathrm{part}} \simeq N_{\mathrm{kk}} \frac{\epsilon}{l}
$$

where $N_{\mathrm{kk}}$ is the average number of kink-kink collisions per oscillation. Values possibly as large as $N_{\mathrm{kk}} \sim O\left(10^{3}\right)$ have been considered in [110] or even as large as $10^{6}$ for the special case of strings with junctions [144], due to kink proliferations [145]. In contrast to the cusp case, the energy radiated per kink-kink collision, $\epsilon$, is independent of the loop size $l$ and we expect $\epsilon \sim \mu^{1 / 2}$.

Upon comparing the power of GW emission in eq. (7) with either eq. (13) or eq. (14), one expects gravitational production to be more efficient than particle production when loops are larger than [52]

$$
l \gtrsim l_{c} \equiv \beta_{c} \frac{\mu^{-1 / 2}}{(\Gamma G \mu)^{2}},
$$

for small-scale structures dominated by cusps, and

$$
l \gtrsim l_{k} \equiv \beta_{k} \frac{\mu^{-1 / 2}}{\Gamma G \mu},
$$

for kink-kink collision domination. $\beta_{\mathrm{c}}$ and $\beta_{\mathrm{k}}$ are numbers which depend on the precise refinement. We assume $\beta_{\mathrm{c}}, \beta_{\mathrm{k}} \sim O(1)$. Therefore, loops with length smaller than the critical value in eq. (15) or eq. (16) are expected to decay into massive radiation before they have time to emit GW, which means that they should be subtracted when computing the SGWB. Equations (15) and (16) are crucial to determine the cutoff frequency, as we discuss in Section 3.4. 


\subsection{Assumptions on the loop distribution}

The SGWB resulting from the emission by CS loops strongly relies on the distribution of loops. In the present section, we introduce the loop-formation efficiency and discuss the assumptions on the loop-production rate, inspired from NG simulations. The loopformation efficiency is computed later, in sec. 4.

Loop-formation efficiency: The SGWB resulting from the emission by CS loops strongly relies on the assumption for the distribution of loops which we now discuss. The equation of motion of a NG string in a expanding universe implies the following evolution equation for the long string energy density, c.f. sec. D

$$
\frac{d \rho_{\infty}}{d t}=-2 H\left(1+\bar{v}^{2}\right) \rho_{\infty}-\left.\frac{d \rho_{\infty}}{d t}\right|_{\text {loop }},
$$

where $\bar{v}$ is the long string mean velocity. The energy loss into loop formation can be expressed as [56]

$$
\left.\frac{d \rho_{\infty}}{d t}\right|_{\text {loop }} \equiv \mu \int_{0}^{\infty} l f(l, t) d l \equiv \frac{\mu}{t^{3}} \tilde{C}_{\mathrm{eff}}
$$

with $f(l, t)$ the number of loops created per unit of volume, per unit of time $t$ and per unit of length $l$ and where we introduced the loop-formation efficiency $\tilde{C}_{\text {eff }}$. The loopformation efficiency $\tilde{C}_{\text {eff }}$ is related to the notation introduced in [21, 22] by

$$
\tilde{C}_{\text {eff }} \equiv \sqrt{2} C_{\text {eff }}
$$

In sec. 4 , we compute the loop-formation efficiency $C_{\text {eff }}$ as a function of the long string network parameters $\bar{v}$ and $L$, which themselves are solutions of the Velocity-dependent One-Scale (VOS) equations.

Only loops produced at the horizon size contribute to the SGWB: As pointed out already a long time ago by $[80,136]$ and more recently in large NG simulations [101], the most numerous loops are the ones of the size of the gravitational backreaction scale

$$
\Gamma G \mu \times t
$$

which acts as a cut-off below which, small-scale structures are smoothened and such that smaller loops can not be produced below that scale. However, it has been claimed that only large loops are relevant for GW [35, 101, 148]. In particular, NG numerical simulations realized by Blanco-Pillado et al. [101] have shown that a fraction $\mathcal{F} \simeq 10 \%$ of the loops are produced with a length equal to a fraction $\alpha \simeq 10 \%$ of the horizon size, and with a Lorentz boost factor $\gamma \simeq \sqrt{2}$. The remaining $90 \%$ of the energy lost by long strings goes into highly boosted smaller loops whose contributions to the GW spectrum are sub-dominant. Under those assumptions, the number of loops, contributing to the SGWB, produced per unit of time can be computed from the total energy flow into loops in eq. (18)

$$
\frac{d n}{d t_{i}}=\left.\frac{\mathcal{F}}{\gamma \mu \alpha t_{i}} \frac{d \rho_{\infty}}{d t}\right|_{\text {loop }},
$$


with $\mathcal{F}=0.1, \gamma=\sqrt{2}$ and $\alpha=0.1$. The latter can be recast as a function of the loop-formation efficiency $\tilde{C}_{\text {eff }}$ defined in eq. (18)

$$
\frac{d n}{d t_{i}}=\mathcal{F} \frac{\tilde{C}_{\text {eff }}\left(t_{i}\right)}{\gamma \alpha t_{i}^{4}}
$$

This is equivalent to choosing the following monochromatic horizon-sized loop-formation function

$$
f\left(l, t_{i}\right)=\frac{\tilde{C}_{\text {eff }}}{\alpha t_{i}^{4}} \delta\left(l-\alpha t_{i}\right) .
$$

The assumptions leading to eq. (22) are the ones we followed for our study and which are also followed by [21, 22]. Our results strongly depend on these assumptions and would be dramatically impacted if instead we consider the model discussed in the next paragraph.

A second population of smaller loops: The previous assumption - that the only loops relevant for the GW signal are the loops produced at horizon size - which is inspired from the NG numerical simulations of Blanco-Pillado et al. [101, 149], is in conflict with the results from Ringeval et al. [110, 126, 150]. In the latter works, the loop production function is derived analytically starting from the correlator of tangent vectors on long strings, within the Polchinski-Rocha (PR) model [151-154]. In the PR model, which has been tested in AH simulations [128], the gravitational back-reaction scale, i.e. the lower cut-off of the loop production function, is computed to be

$$
\Upsilon(G \mu)^{1+2 \chi} \times t
$$

with $\Upsilon \simeq 10$ and $\chi \sim 0.25$. Consequently, the gravitational back-reaction scale in the PR model is significantly smaller than the usual gravitational back-reaction scale, commonly assumed to match the gravitational radiation scale, $\Gamma G \mu t$. Therefore, the model of Ringeval et al. predicts the existence of a second population of smaller loops which enhances the GW spectrum at high frequency by many orders of magnitude [110]. However, as raised by [155], the model of Ringeval et al. predicts the amount of longstring energy converted into loops, to be $\sim 200$ times larger than the one computed in the numerical simulations of Blanco-Pillado et al. [101]. These discrepancies between Polchinski-Rocha analytical modeling and Nambu-Goto numerical simulations remain to be understood. 


\subsection{The gravitational-wave spectrum}

For our study, we compute the GW spectrum observed today generated from CS as follows (see app. B for a derivation)

$$
\Omega_{\mathrm{GW}}(f) \equiv \frac{f}{\rho_{c}}\left|\frac{d \rho_{\mathrm{GW}}}{d f}\right|=\sum_{k} \Omega_{\mathrm{GW}}^{(k)}(f)
$$

where

$$
\Omega_{\mathrm{GW}}^{(k)}(f)=\frac{1}{\rho_{c}} \cdot \frac{2 k}{f} \cdot \frac{(0.1) \Gamma^{(k)} G \mu^{2}}{\alpha(\alpha+\Gamma G \mu)} \int_{t_{F}}^{t_{0}} d \tilde{t} \frac{C_{\mathrm{eff}}\left(t_{i}\right)}{t_{i}^{4}}\left[\frac{a(\tilde{t})}{a\left(t_{0}\right)}\right]^{5}\left[\frac{a\left(t_{i}\right)}{a(\tilde{t})}\right]^{3} \Theta\left(t_{i}-t_{F}\right) \Theta\left(t_{i}-\frac{l_{*}}{\alpha}\right),
$$

with

$\mu, G, \rho_{c} \equiv$ string tension, Newton constant, critical density

$\Theta \equiv$ Heaviside function

$a \equiv$ scale factor of the universe

(we solve the full Friedmann equation for a given energy density content),

$\Gamma \equiv$ gravitational loop-emission efficiency, $(\Gamma \simeq 50$ [149])

$\Gamma^{(k)} \equiv$ Fourier modes of $\Gamma$, dependent on the loop small-scale structures, $\left(\Gamma^{(k)} \propto k^{-4 / 3}\right.$ for cusps, e.g. [36]),

$C_{\text {eff }} \equiv$ loop-production efficiency, defined in eq. (33),

( $C_{\text {eff }}$ is a function of the long-string mean velocity $\bar{v}$ and correlation length $\xi$,

both computed upon integrating the VOS equations, c.f. sec. 4)

$\alpha \equiv$ loop length at formation in unit of the cosmic time, $(\alpha \simeq 0.1)$

(we consider a monochromatic, horizon-sized loop-formation function, c.f. 3.2),

$\tilde{t} \equiv$ the time of GW emission,

$f \equiv$ observed frequency today

(related to frequency at emission $\tilde{f}$ through $f a\left(t_{0}\right)=\tilde{f} a(\tilde{t})$,

related to loop length $l$ through $\tilde{f}=2 k / l$,

related to the time of loop production $t_{i}$ through $\left.l=\alpha t_{i}-\Gamma G \mu\left(t-t_{i}\right)\right)$,

$t_{i} \equiv$ the time of loop production,

(related to observed frequency and emission time $\tilde{t}$ through

$\left.t_{i}(f, \tilde{t})=\frac{1}{\alpha+\Gamma G \mu}\left[\frac{2 k}{f} \frac{a(\tilde{t})}{a\left(t_{0}\right)}+\Gamma G \mu \tilde{t}\right]\right)$.

$t_{0} \equiv$ the time today,

$t_{F} \equiv$ the time of cosmic-string network formation,

$l_{*} \equiv l_{\mathrm{c}}$ for cusps and $l_{\mathrm{k}}$ for kinks in eq. (15) and eq. (16)

(critical length below which the emission of massive radiation

is more efficient than the gravitational emission, c.f. sec. 3.1).

Fig. 2 shows the GW spectrum computed with eq.(25). We indicate separately the contributions from the emission occurring before and after the matter-radiation equality. 


$$
\mathrm{G} \mu=10^{-11}, \Gamma=50, \alpha=0.1
$$

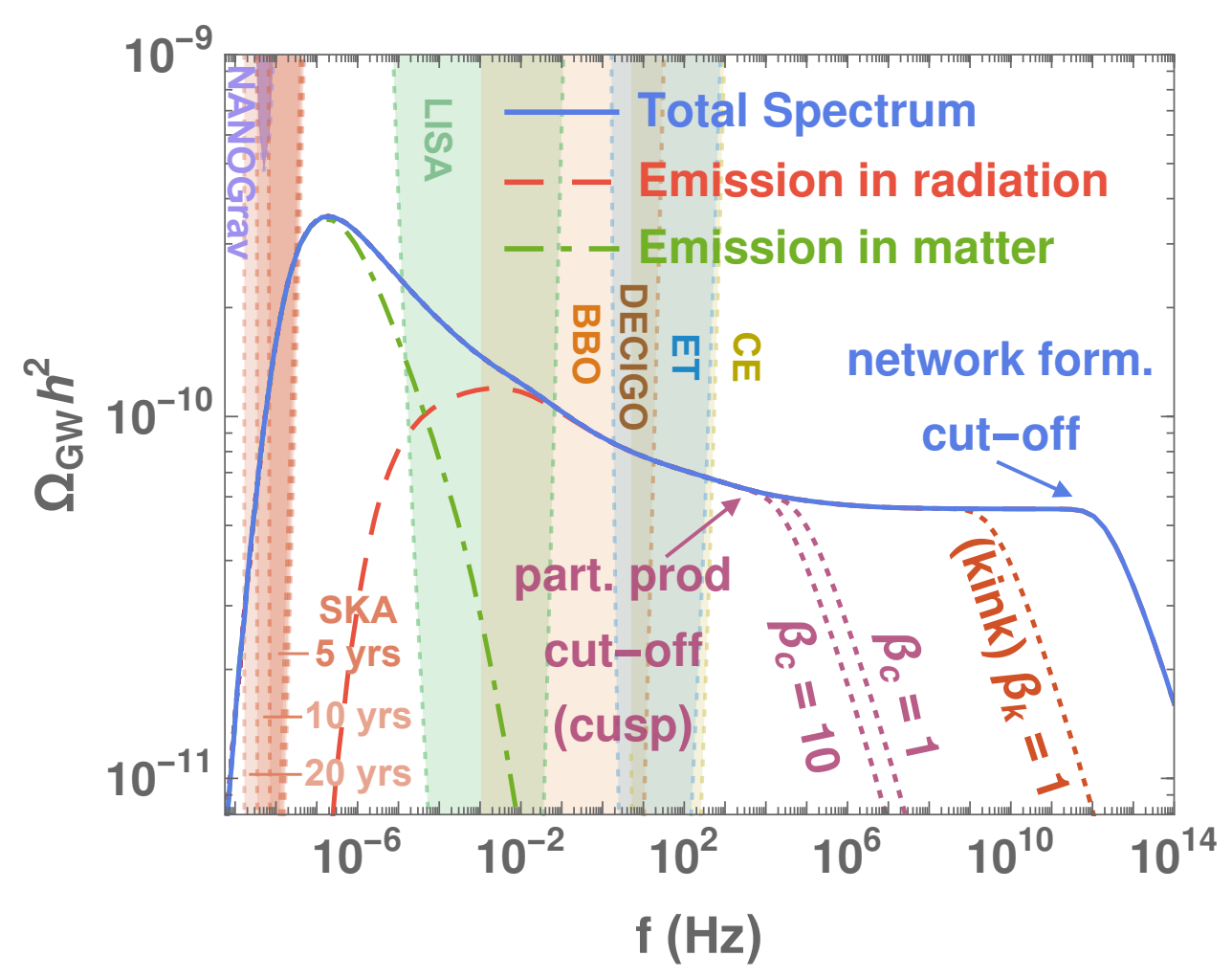

Figure 2: $G W$ spectrum from the scaling cosmic-string network evolving in a standard cosmology. Contributions from $G W$ emitted during radiation and matter era are shown with red and green dashed lines respectively. The high-frequency cut-offs correspond to the time of formation of the network or when massive particle production dominates over gravitational emission, for kink or cusp-dominated small-scale structures. The cut-offs are described by two Heaviside functions in the master formula in eq. (25). Colored regions indicate the integrated power-law sensitivity of future experiments, as described in app. F.

One can see that loops emitting during the radiation era contribute to a flat spectrum whereas loops emitting during the matter era lead to a slope decreasing as $f^{-1}$.

In a nutshell, the frequency dependence of the GW spectrum receives two contributions, a red-tilt coming from the redshift of the GW energy density and a blue-tilt coming from the loop-production rate $\propto t_{i}^{-4}$. On the one hand, the higher the frequency the earlier the GW emission, so the larger the redshift of the GW energy density and the more suppressed the spectrum. On the other hand, high frequencies correspond to loops formed earlier, those being more numerous, this increases the GW amplitude. Interestingly, during radiation-domination the two contributions exactly cancel such that the spectrum is flat. During matter domination, because of the faster expansion rate, the tilt induced by the redshift factor takes over the tilt induced by the loop-production rate, hence leading to a slope decreasing as $f^{-1}$. Thus loops created during the late standard matter era give rise to the peak at low frequency. Conversely, during kination domination, a lower expansion rate implies a slope increasing as $f^{1}$. A more quantitative analysis is given in [22]. 


\subsection{The frequency - temperature relation}

Relation between frequency of observation and temperature of loop formation: In app. $\mathrm{C}$, we derive the relation between a detected frequency $f$ and the temperature of the universe when the loops, mostly responsible for $f$, are formed

$$
f=\left(6.7 \times 10^{-2} \mathrm{~Hz}\right)\left(\frac{T}{\mathrm{GeV}}\right)\left(\frac{0.1 \times 50 \times 10^{-11}}{\alpha \Gamma G \mu}\right)^{1 / 2}\left(\frac{g_{*}(T)}{g_{*}\left(T_{0}\right)}\right)^{1 / 4} .
$$

We emphasize that eq. (27) is very different from the relation obtained in the case of GW generated by a first-order cosmological phase transition. In the latter case, the emitted frequency corresponds to the Hubble scale at $T_{p}[1]$

$$
f=\left(19 \times 10^{-3} \mathrm{mHz}\right)\left(\frac{T_{p}}{100 \mathrm{GeV}}\right)\left(\frac{g_{*}\left(T_{p}\right)}{100}\right)^{1 / 4} .
$$

In the case of cosmic strings, instead of being set by the Hubble scale at the loop-formation time $t_{i}$, the emitted frequency is further suppressed by a factor $(\Gamma G \mu)^{-1 / 2}$, which we now explain. From the scaling law $\propto t_{i}^{-4}$ of the loop-production function in eq.(22), one can understand that the most numerous population of emitting loops at a given time $\tilde{t}$ is the population of loops created at the earliest epoch. They are the oldest loops ${ }^{2}$. Hence, a loop created at time $t_{i}$ contributes to the SGWB much later, at a time given by the loop half-lifetime $\tilde{t}_{\mathrm{M}}=\alpha t_{i} / 2 \Gamma G \mu$, c.f. eq. (11). Therefore, the emitted frequency is dispensed from the redshift factor $a\left(\tilde{t}_{\mathrm{M}}\right) / a\left(t_{i}\right)=\left(\tilde{t}_{\mathrm{M}} / t_{i}\right)^{1 / 2} \sim(\Gamma G \mu)^{-1 / 2}$, and so, is higher. See app. $\mathrm{C}$ and its fig. 24 for more details.

The detection of a non-standard cosmology: During a change of cosmology, e.g a change from a matter to a radiation-dominated era, the long-string network evolves from one scaling regime to the other. The response of the network to the change of cosmology is quantified by the VOS equations, which are presented in sec. 4. As a result of the transient evolution towards the new scaling regime, the turning-point frequency eq. (82) associated to the change of cosmology is lower in VOS than in the scaling network. The detection of a turning-point in a GW spectrum from CS by a future interferometer would be a smoking-gun signal for non-standard cosmology. Particularly, in fig. 21, we show that LISA can probe a non-standard era ending around the QCD scale, ET/CE can probe a non-standard era ending around the $\mathrm{TeV}$ scale whereas DECIGO/BBO can probe the intermediate range. We show particular examples of long-lasting era in sec. 6. We focus on the particular case of a short matter era in sec. 7 and a short inflation era in sec. 8, respectively. In the latter case, the turning-point frequency is even further decreased due to the string stretching which we explain in the next paragraph.

The detection of a non-standard cosmology (intermediate-inflation case): If the universe undergoes a period of inflation lasting $N_{e}$ e-folds, the correlation length of the network is stretched outside the horizon. After inflation, the network achieves a long transient regime lasting $\sim N_{e}$ other e-folds until the correlation length re-enters the horizon. Hence, the turning-point frequency in the GW spectrum, c.f. eq. (52), receives a $\exp N_{e}$ suppression compared to eq. (27) due to the duration of the transient. We give more details in sec. 8.

\footnotetext{
${ }^{2}$ Note that they are also the smallest loops, with a length given by the gravitational radiation scale $\Gamma G \mu t$.
} 
Cut-off frequency from particle production: As discussed in the sec. 3.1, particle production is the main decay channel of loops shorter than

$$
l_{*}=\beta_{m} \frac{\mu^{-1 / 2}}{(\Gamma G \mu)^{m}},
$$

where $m=1$ or 2 for loops kink-dominated or cusp-dominated, respectively, and $\beta_{m} \sim$ $\mathcal{O}(1)$. The corresponding characteristic temperature above which loops, decaying preferentially into particles, are produced, is

$T_{*} \simeq \beta_{m}^{-1 / 2} \Gamma^{m / 2} \sqrt{\alpha}(G \mu)^{(2 m+1) / 4} M_{\mathrm{pl}} \simeq \begin{cases}(0.2 \mathrm{EeV}) \sqrt{\frac{\alpha}{0.1}} \sqrt{\frac{1}{\beta_{c}}}\left(\frac{G \mu}{10^{-15}}\right)^{3 / 4} & \text { for kinks }, \\ (1 \mathrm{GeV}) \sqrt{\frac{\alpha}{0.1}} \sqrt{\frac{1}{\beta_{k}}}\left(\frac{G \mu}{10^{-15}}\right)^{5 / 4} & \text { for cusps. }\end{cases}$

We have used $l_{*}=\alpha t_{i}, H=1 /\left(2 t_{i}\right)$ and $\rho_{\text {rad }}=3 M_{\mathrm{pl}}^{2} H^{2}$. Upon using the frequencytemperature correspondence in eq. (27), we get the cut-off frequencies due to particle production

$$
f_{*} \simeq \begin{cases}(1 \mathrm{GHz}) \sqrt{\frac{1}{\beta_{c}}}\left(\frac{G \mu}{10^{-15}}\right)^{1 / 4} & \text { for kinks } \\ (31 \mathrm{~Hz}) \sqrt{\frac{1}{\beta_{k}}}\left(\frac{G \mu}{10^{-15}}\right)^{3 / 4} & \text { for cusps. }\end{cases}
$$

and which we show in most of our plots with dotted red and purple lines. Particularly, in fig. 21, we see that particle production in the cusp-dominated case would start suppressing the GW signal in the ET/CE windows for string tension lower than $G \mu \lesssim 10^{-15}$. However, in the kink-dominated case, the spectrum is only impacted at frequencies much higher than the interferometer windows.

\subsection{The astrophysical foreground}

Crucial for our analysis is the assumption that the stochastic GW foreground of astrophysical origin can be substracted.

LIGO/VIRGO has already observed three binary black hole (BH-BH) merging events [156-158] during the first 4-month observing run O1 in 2015, and seven additional BH$\mathrm{BH}$ [159-162] as well as one binary neutron star (NS-NS) [163] merging events during the second 9-month observing run O2 in 2017. And more events might still be discovered in the $\mathrm{O} 2$ data [164]. According to the estimation of the NS merging rate following the detection of the first (and unique up to now) NS-NS merger event GW170817, NSNS stochatisc background may be detectable after a 20-month observing run with the expected LIGO/VIRGO design sensistivity in 2022+ and in the most optimistic scenario, it might be detectable after 18-month of the third observing run $\mathrm{O} 3$ who began in April, $1^{\text {st }}, 2019$ [165]. Hence, one might worry about the possibility to distinguish the GW SGWB sourced by CS from the one generated by the astrophysical foreground. However, in the $\mathrm{BBO}$ and $\mathrm{ET} / \mathrm{CE}$ windows, the $\mathrm{NS}$ and $\mathrm{BH}$ foreground might be substracted with respective reached sensibilities $\Omega_{\mathrm{GW}} \simeq 10^{-15}$ [166] and $\Omega_{\mathrm{GW}} \simeq 10^{-13}$ [167]. In the LISA window, the binary white dwarf (WD-WD) foreground dominates over the NS-NS and BH-BH foregrounds [168-170]. The WD-WD galactic foreground, one order 
of magnitude higher than the WD-WD extragalactic [171], might be substracted with reached sensibility $\Omega_{\mathrm{GW}} \simeq 10^{-13}$ at $\operatorname{LISA}[172,173]$. Hence, in the optimistic case where the foreground can be removed and the latter sensibility are reached one might be able to distinguished the signal sourced by CS from the one generated by the astrophysical foreground. Furthermore, the GW spectrum generated by the astrophysical foreground increased with frequency as $f^{2 / 3}$ [174], which is different from the GW spectrum generated by CS during radiation (flat), matter $f^{-1}$, inflation $f^{-1}$ or kination $f^{1}$.

\section{The Velocity-dependent One-Scale model}

The master formula (25) crucially depends on the loop-production efficiency encoded in $C_{\text {eff }}$. In this section, we discuss its derivation within the framework of the Velocitydependent One-Scale (VOS) model.

\subsection{The loop-production efficiency}

In a correlation volume $L^{3}$, a segment of length $L$ must travel a distance $L$ before encountering another segment. $L$ is the correlation length of the long-string network. The collision rate, per unit of volume, is $\frac{\bar{v}}{L} \cdot \frac{1}{L^{3}} \sim \frac{\bar{v}}{L^{4}}$ where $\bar{v}$ is the long-string mean velocity. At each collision forming a loop, the network looses a loop energy $\mu L=\rho_{\infty} L^{3}$. Hence, the loop-production energy rate can be written as [78]

$$
\left.\frac{d \rho_{\infty}}{d t}\right|_{\text {loop }}=\tilde{c} \bar{v} \frac{\rho_{\infty}}{L}
$$

where one can compute $\tilde{c}=0.23 \pm 0.04$ from NG simulations in expanding universe [175]. $\tilde{c}$ is the only free parameter of the VOS model. Hence, the loop-formation efficiency, defined in eq. (18), can be expressed as a function of the long-string parameters, $\bar{v}$ and $\xi \equiv L / t$

$$
\tilde{C}_{\text {eff }} \equiv \sqrt{2} C_{\text {eff }}(t)=\frac{\tilde{c} \bar{v}(t)}{\xi^{3}(t)} .
$$

In app. E, we discuss how our results are changed when considering a recent extension of the VOS model with more free parameters, fitted on Abelian-Higgs field theory numerical simulations [176], and taking into account the emission of massive radiation. Basically, the loop-formation efficiency $C_{\text {eff }}$ is only decreased by a factor $\sim 2$. In the following, we derive $\bar{v}$ and $\xi$ as solutions of the VOS equations.

\subsection{The VOS equations}

The VOS equations describe the evolution of a network of long strings in term of the mean velocity $\bar{v}$ and the correlation length $\xi=L / t[175,177-179]$. The latter is defined through the long string energy density $\rho_{\infty} \equiv \mu / L^{2}$. Starting from the equations of motion of the NG string in a FRW universe, we can derive the so-called VOS equations (see app. D for a derivation)

$$
\begin{aligned}
& \frac{d L}{d t}=H L\left(1+\bar{v}^{2}\right)+\frac{1}{2} \tilde{c} \bar{v}, \\
& \frac{d \bar{v}}{d t}=\left(1-\bar{v}^{2}\right)\left[\frac{k(\bar{v})}{L}-2 H \bar{v}\right],
\end{aligned}
$$


where

$$
k(\bar{v})=\frac{2 \sqrt{2}}{\pi}\left(1-\bar{v}^{2}\right)\left(1+2 \sqrt{2} \bar{v}^{3}\right) \frac{1-8 \bar{v}^{6}}{1+8 \bar{v}^{6}},
$$

is the so-called momentum parameter and is a measure of the deviation from the straight string, for which $k(\bar{v})=1$ [179]. The first VOS equation describes the evolution of the long string correlation length under the effect of Hubble expansion and loop chopping. The second VOS equation is nothing more than a relativistic generalization of Newton's law where the string is accelerated by its curvature $1 / L$ but is damped by the Hubble expansion after a typical length $H^{-1}$.

Numerical simulations [90-94] have shown that a network of long strings is first subject to a transient regime before reaching a scaling regime, in which the long string mean velocity $\bar{v}$ is constant and the correlation length grows linearly with the Hubble horizon $L=\xi t$. The values of the quantities $\bar{v}$ and $\xi$ depend on the cosmological background, namely the equation of state of the universe. Hence, when passing from a cosmological era 1 to era 2, the network accomplishes a transient evolution from the scaling regime 1 to the scaling regime 2. We use the VOS equations to compute the time evolution of $\bar{v}$ and $\xi$ during the change of cosmology and then compute their impact on the CS SGWB.

\subsection{Scaling regime solution and beyond}

Scaling solution vs VOS solution: Fig. 3 shows the evolutions of $\xi, \bar{v}$, and $C_{\text {eff }}$, from solving the VOS equations in eq. (34) with three equations of state, matter, radiation and kination. Regardless of the initial-condition choice, the network approaches a scaling solution where all parameters become constant. The energy scale of the universe has to decrease by some 4 orders of magnitude before reaching the scaling regime after the network formation. For a cosmological background evolving as $a \propto t^{2 / n}$ with $n \geq 2$, the scaling regime solution is

$$
\xi=\text { constant and } \bar{v}=\text { constant }
$$

with

$$
\text { with } \quad \xi=\frac{n}{2} \sqrt{\frac{k(\bar{v})[k(\bar{v})+\tilde{c}]}{2(n-2)}} \text { and } \quad \bar{v}=\sqrt{\frac{n}{2} \frac{k(\bar{v})}{[k(\bar{v})+\tilde{c}]}\left(1-\frac{2}{n}\right)} .
$$

In order to fix the notation used in our plots, we define

- (Instantaneous) scaling network: The loop-formation efficiency $C_{\text {eff }}$, defined in eq. (33), is taken at its steady state value, given by eq. (38). In particular for matter, radiation and kination domination, one has

$$
C_{\text {eff }} \simeq 0.39,5.4,29.6 \quad \text { for } n=3,4,6 \text {. }
$$

During a change of era $1 \rightarrow 2, C_{\text {eff }}$ is assumed to change instantaneously from the scaling regime of era 1 to the scaling regime of era 2 . This is the assumption adopted in [21, 22].

- VOS network: The loop-formation efficiency $C_{\text {eff }}$, defined in eq. (33), is computed upon integrating the VOS equations in eq. (34). During a change of cosmology, the long-string network experiences a transient regime. 

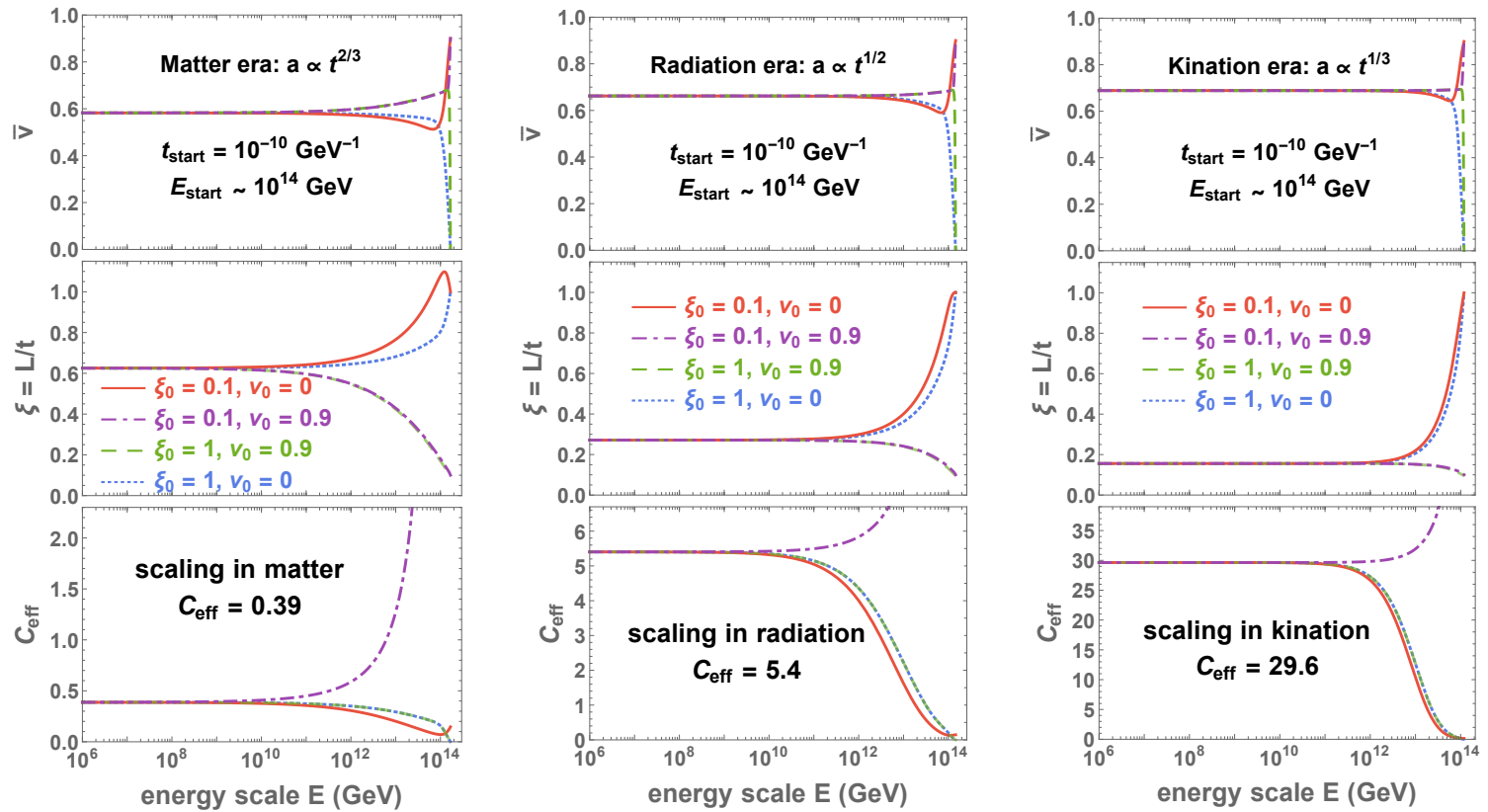

Figure 3: Cosmic-string network evolving in the one-component universe with energy density $\rho \sim a^{-n}$ where $n=3,4$ and 6 correspond to matter, radiation and kination, respectively. The long-string-network mean velocity $\bar{v}$, the correlation length $\xi$ and the corresponding loopproduction efficiency $C_{\text {eff }}$ reach the scale-invariant solutions after the Hubble expansion rate has dropped by 2 orders of magnitude, independently of the initial conditions.

Beyond the scaling regime in standard cosmology: In fig. 4 and fig. 5, we compare the GW spectra and the $C_{\text {eff }}$ evolution, obtained with a scaling and VOS network. They are quite similar. The main difference arises from the change in relativistic degrees of freedom near the QCD confining temperature and from the matter-radiation transition. In contrast, predictions differ significantly when considering non-standard cosmology.

Beyond the scaling regime in non-standard cosmology: In fig. 8, in dashed vs solid, we compare the loop-production efficiency factor $C_{\text {eff }}$ and the corresponding GW spectra for a scaling network and for a VOS network. The VOS frequency of the turning point due to the change of cosmology is shifted to a lower frequency by a factor $\sim 22.5$ with respect to the corresponding scaling frequency. The shift results from the extra-time needed by the network to achieve its transient evolution to the new scaling regime. In the rest of this work, we go beyond the instantaneous scaling approximation used in [21, 22].

\section{$5 \quad$ Standard cosmology}

\subsection{The cosmic expansion}

The SGWB from CS, c.f. master formula in eq. (25), depends on the cosmology through the scale factor $a$. We compute the later upon integrating the Friedmann equation

$$
H^{2}=\frac{\rho}{3 M_{\mathrm{pl}}^{2}}
$$


for a given energy density $\rho$. In the standard $\Lambda$ CDM scenario, the universe is first dominated by radiation, then a matter era, and finally the cosmological constant so that we can write the energy density as

$$
\rho_{\mathrm{ST}, 0}(a)=\rho_{r, 0} \Delta_{R}\left(T(a), T_{0}\right)\left(\frac{a}{a_{0}}\right)^{4}+\rho_{m, 0}\left(\frac{a}{a_{0}}\right)^{3}+\rho_{k, 0}\left(\frac{a}{a_{0}}\right)^{2}+\rho_{\Lambda, 0},
$$

where $r, m, k$ and $\Lambda$ denote radiation, matter, curvature, and the cosmological constant, respectively. We take $\rho_{i}=\Omega_{i} h^{2} 3 M_{\mathrm{pl}}^{2} H_{0}^{2}$, where $H_{0}=100 \mathrm{~km} / \mathrm{s} / \mathrm{Mpc}, \Omega_{r} h^{2} \simeq 4.2 \times 10^{-5}$, $\Omega_{\mathrm{m}} h^{2} \simeq 0.14, \Omega_{k} \simeq 0, \Omega_{\Lambda} h^{2} \simeq 0.31$ [180]. The presence of the function

$$
\Delta_{R}=\left(\frac{g_{*}(T)}{g_{*}\left(T_{0}\right)}\right)\left(\frac{g_{* s}\left(T_{0}\right)}{g_{* s}(T)}\right)^{4 / 3},
$$

comes from imposing the conservation of the comoving entropy $g_{* s} T^{3} a^{3}$. We discuss the possibility of adding an extra source of energy density in the next sections, long matter/kination in sec. 6, intermediate matter in sec. 7 and intermediate inflation in sec. 8.

\subsection{Gravitational wave spectrum}

Fig. 4 shows the dependence of the spectrum on the string tension. The amplitude decreases with $G \mu$ due to the lower energy stored in the strings. Moreover, at lower $G \mu$, the loops decaying slower, the GW are emitted later, implying a lower redshift factor and a global shift of the spectrum to higher frequencies. This figure also shows how the change in SM relativistic degrees of freedom introduces a small red-tilt which suppresses the spectrum by a factor $\sim 2.5$ at high frequencies.

\subsection{Deviation from the scaling regime}

Fig. 5 shows how the loop-formation efficiency $C_{\text {eff }}$ varies during the change of SM relativistic degrees of freedom and the matter-radiation equality, upon solving the VOS equations, c.f. sec. 4. We see the associated corrections to the spectrum in fig. 4, and which were already pointed out in [23]. The spectrum is enhanced at low frequencies because more loops are produced than when assuming that the matter era is reached instantaneously, c.f. fig. 5 .

\subsection{Beyond the NG approximation}

Fig. 4 shows the possibility of a cut-off at high frequencies due to particle production, for two different assumptions regarding the loop small-scale structures: cusps or kinks domination, c.f. sec. 3.1. Above these frequencies, loops decays into massive radiation before they have time to emit GW. For kinky loops, the cut-off is outside any futureplanned observational bands, while for cuspy loops, the cut-off might be in the observed windows for $G \mu \lesssim 10^{-15}$.

\subsection{Initial network configuration}

Fig. 6 shows how the spectrum depends on the choice of initial conditions $\bar{v}_{0}, \xi_{0}$. As expected, only the region near the high-frequency cut-off, corresponding to loops created 

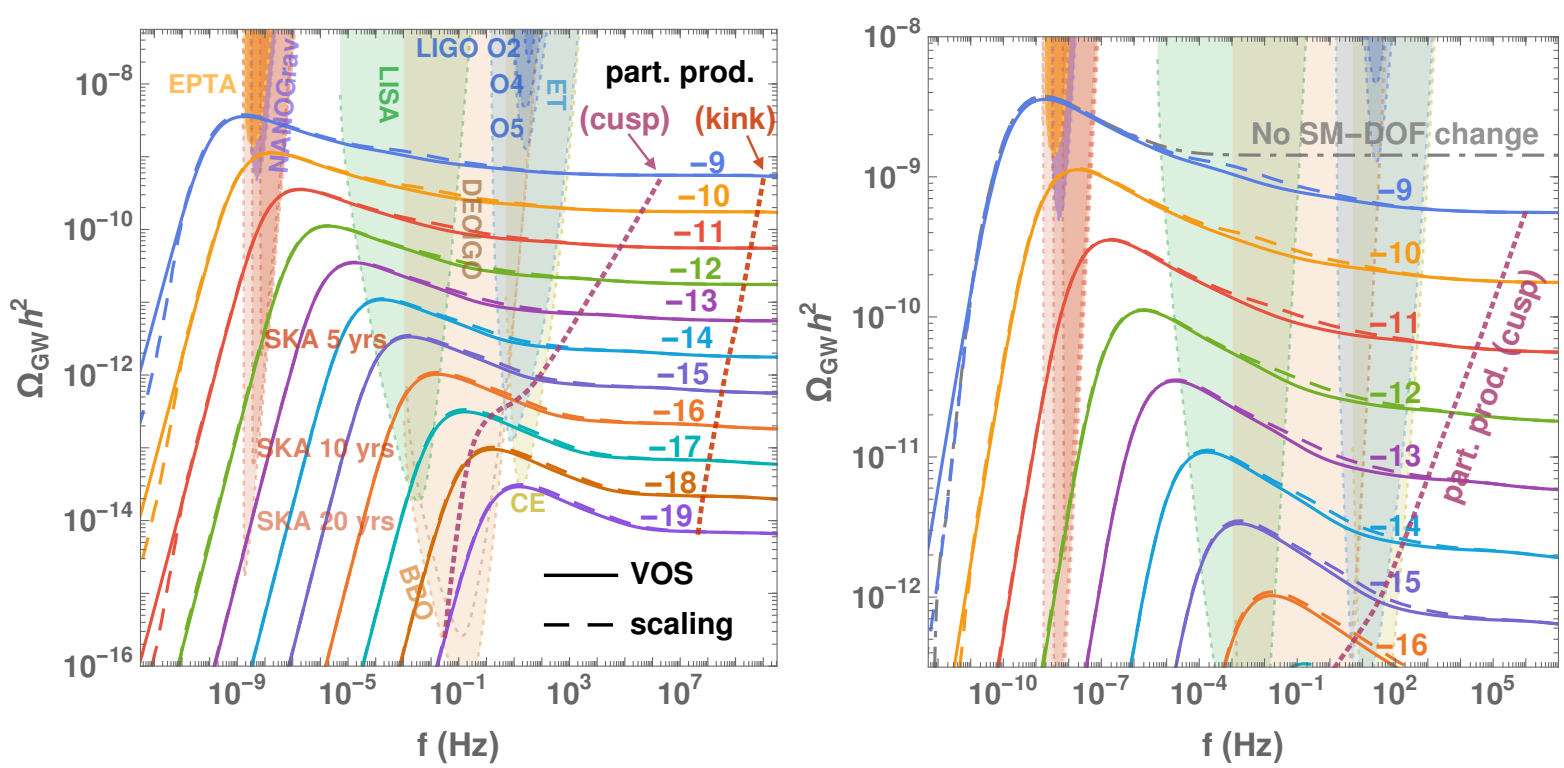

Figure 4: Left: $G W$ spectra from cosmic strings assuming either the scaling or VOS network, c.f. sec. 4.3, evolving in the standard cosmological background. Dotted lines show the spectral cut-offs expected due to particle production, c.f. sec. 3.4, which depend on the nature of the loop small-scale structures: cusp or kink-dominated. Right: The zoom-in plot of the left panel shows the effects from the change of SM degrees of freedom on the scaling and VOS networks.

$$
\begin{aligned}
& \text { Loop-Production Coefficient } C_{\text {eff }} \\
& \qquad \mathrm{G} \mu \sim 10^{-9} \Rightarrow T_{F} \sim 10^{15} \mathrm{GeV}
\end{aligned}
$$

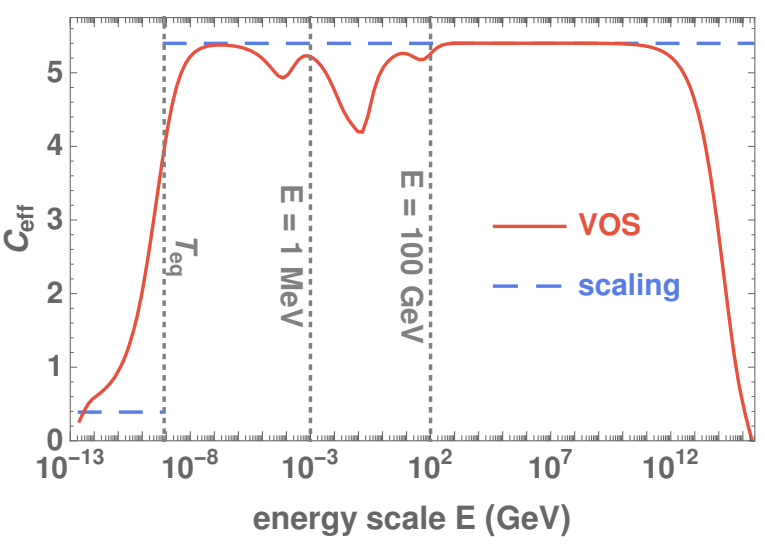

Figure 5: Comparison of the loop-production efficiency under the scaling assumption, where the attractor solution of the VOS equations is assumed to be reached instantaneously, and under the VOS assumptions, where one integrates the VOS equations. A standard cosmology is assumed.

just after the network formation, is impacted. We see that the smaller/larger $\xi_{0} / \bar{v}_{0}$, the higher the bump. Such initial values lead to an overproduction of loops during the initial transient regime and to an enhancement of the spectrum. The impact of $\xi_{0}$ is stronger than the one of $\bar{v}_{0}$ because the loop-production efficiency scales as $C_{\text {eff }} \propto \bar{v} / \xi^{3}$.

Note that the frequency of the bump is independent of $G \mu$. This can be understood upon plugging the temperature when the network is formed, $T_{F} \sim m_{\mathrm{pl}} \sqrt{G \mu}$ into the $(f-T)$-correspondence formula in eq. (27). Also note that at such a high temperature, the friction of the strings with the plasma might play a major role [181]. 

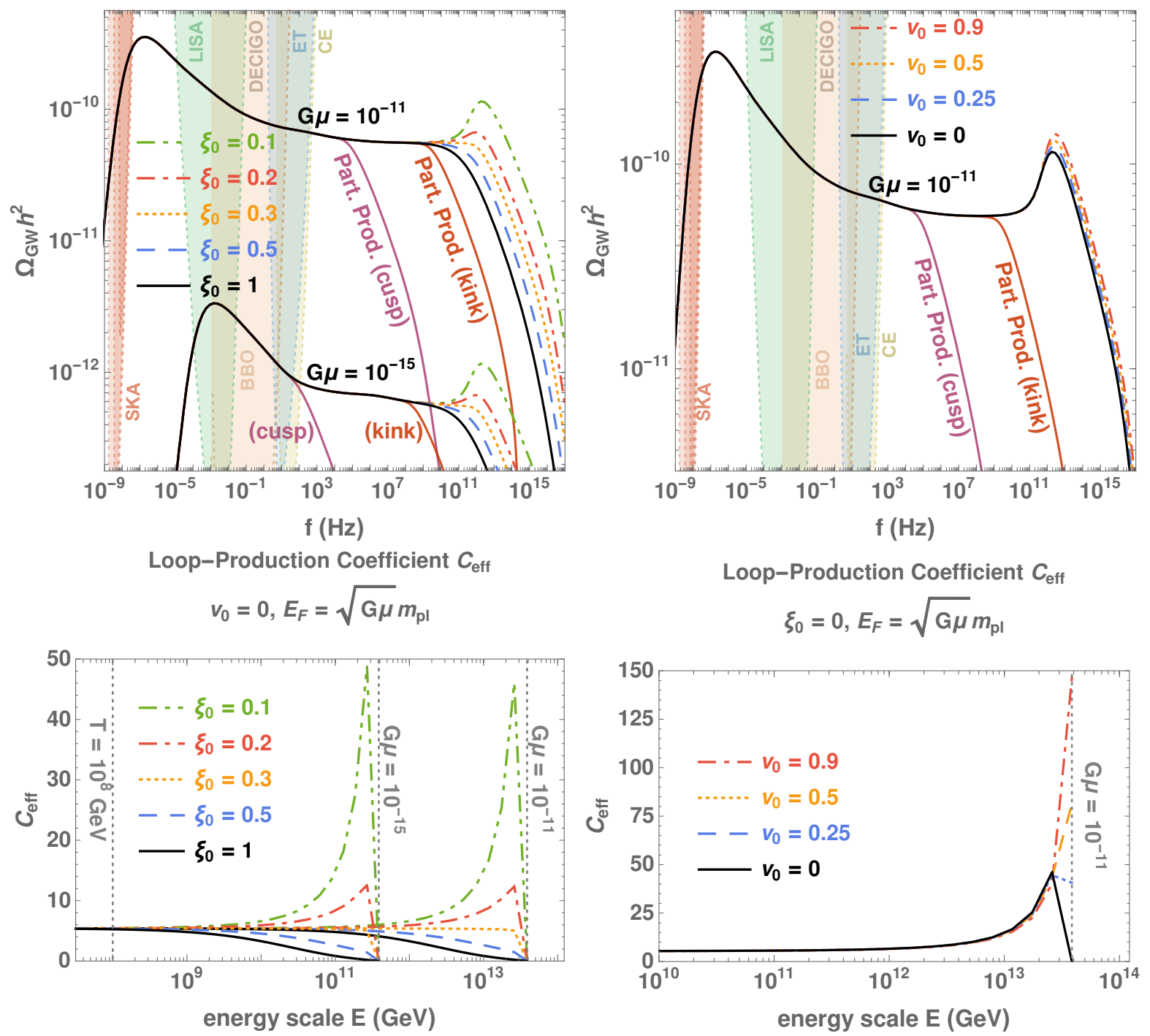

Figure 6: Top: $G W$ spectra assuming a standard cosmology. The network is formed at the temperature specified by the string tension: $T_{F} \sim m_{p l} \sqrt{G \mu}$. Initial conditions with a fixed initial mean velocity $\bar{v}_{0}$ (left) and a fixed initial correlation length scale $\xi_{0}$ (right) are applied. Bottom: The bumps at high frequencies come from the over-production of loops right after the network formation when $\xi / \bar{v}$ are taken smaller/larger than their scaling values.

The high-frequency bump could be a probe of the nature of the PT in the early universe, e.g. the initial correlation length, or a probe of the plasma-string interaction. This could be in principle a motivation for high-frequency GW experiments. However, the loops which would contribute to such high-frequency GW, might rather decay into particles, c.f. solid purple and red line in fig. 6 .

In the next three sections, we will study the impact of different non-standard cosmologies on the SGWB from cosmic strings. Each cosmological history not only yields a distinct value for the scale factor of the universe today, $a_{0}$, thus a different amount of redshifting of gravitational waves in eq. (26), but also a distinct loop-production rate $\propto C_{\text {eff }} / t_{i}^{4}$ due to a different formation time $t_{i}$ and a different loop-production efficiency $C_{\text {eff }}$. In Sec. 6, we assume that the radiation era was preceded by a long period of either 
matter domination or kination all the way after inflation. In Sec. 7 and Sec. 8, we assume instead some short eras of either matter domination or inflation, inside the radiation era.

\section{Long-lasting matter or kination era}

\subsection{The non-standard scenario}

In this section, we consider the presence of a matter or kination-dominated era which starts just after the end of inflation, when the total energy density is $\rho_{\text {start }}=\rho_{\text {inflation }}$, and ends much later, at $\rho_{\text {end }}$, when it becomes supplanted by the standard radiationdominated era. At the end of the non-standard era, the temperature of the universe is $T_{\Delta}$. The energy density profile, sketched in fig. 7 , is given by

$$
\rho_{\text {tot }}(a)= \begin{cases}\rho_{\text {start }}\left(\frac{a_{\text {start }}}{a}\right)^{n}+\rho_{\text {late }}(a) & \text { for } \rho_{\text {start }}>\rho>\rho_{\text {end }}, \\ \rho_{\text {end }} \Delta_{R}\left(T_{\text {end }}, T\right)\left(\frac{a_{\text {end }}}{a}\right)^{4}+\rho_{\text {late }}(a) & \text { for } \rho<\rho_{\text {end }},\end{cases}
$$

where $\quad \rho_{\text {start }}, \rho_{\text {end }} \equiv$ the starting and ending energy density of the non-standard cosmology,

$\rho_{\text {late }} \equiv$ the standard-cosmology energy density dominating at late times, e.g. the standard matter density, and cosmological constant.

$\Delta_{R}$ is given in eq.(42).

Non-standard cosmo. before rad. era

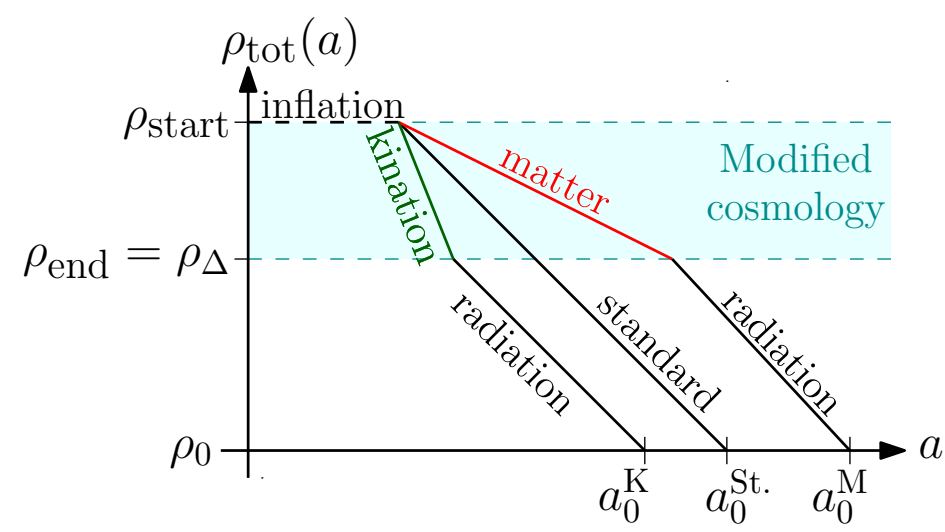

Figure 7: Evolution of the energy density assuming a matter ( $M)$ and kination (K) era after inflation and before the radiation era. 'St' refers to standard cosmology. We suppose that the cosmic string network forms at the end of inflation with tension given by $G \mu \sim\left(\rho_{\text {start }}^{1 / 4} / m_{p l}\right)^{2}$ (for instance the CS network can form through non-thermal dynamical symmetry breaking [182189]).

\subsection{Impact on the spectrum: a turning-point}

The resulting GW spectra are shown in fig. 8 for long-lasting kination and matter eras starting at $E_{\text {start }}=m_{p l} \sqrt{G \mu}$ and ending at $E_{\text {end }}=E_{\Delta}=100 \mathrm{GeV}$ with duration

$$
r \equiv\left(\frac{\rho_{\text {start }}}{\rho_{\text {end }}}\right)^{1 / 4} \equiv\left(\frac{E_{\text {start }}}{E_{\Delta}}\right) \simeq 10^{11} .
$$




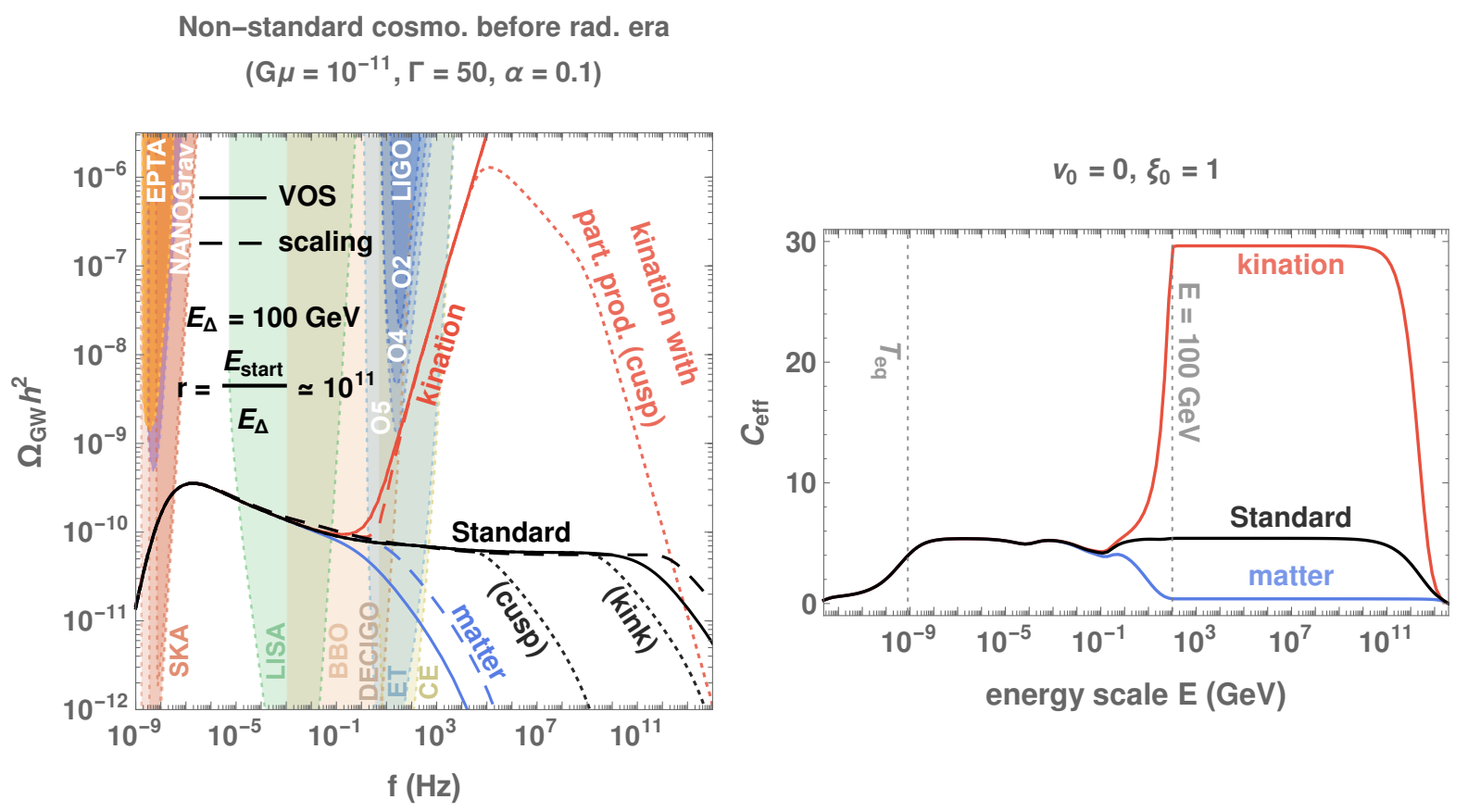

Figure 8: Left: $G W$ spectra from cosmic strings assuming either the scaling (dashed) or the VOS network (solid), c.f. sec. 4.3, evolved in the presence of a non-standard era, either matter (blue) or kination-dominated (red), before the standard radiation era. The transient VOS evolution of the long-string network during the change of cosmology shifts the turning-point towards lower frequencies by $\mathcal{O}(25)$. The cut-offs due to particle production, c.f. sec. 3.4, are shown with dotted lines. Right: The evolution of the loop-production efficiency for each cosmological background shows that the scaling solution is reached after a transient evolution corresponding to the Hubble rate dropping by on order of magnitude. The slower the expansion rate a $\propto t^{2 / n}$, the slower the dilution of the long-string energy density $\rho_{\infty} \propto a^{-2}$ and the higher the needed loop-production efficiency $C_{\text {eff }}$ in order to reach the scaling regime $\rho_{\infty} \propto t^{-2}$.

For kination, the slower expansion of the universe means that loops are produced earlier when the loop-production is more efficient, c.f. eq. (22), which enhances the spectrum. For matter domination, we have the opposite behavior and the spectrum is suppressed.

The turning-point frequency: A key observable is the frequency above which the GW spectrum differs from the one obtained in standard cosmology. This is the so-called turning-point frequency $f_{\Delta}$. It corresponds to the redshifted-frequency emitted by the loops created during the change of cosmology at the temperature $T_{\Delta}$. In the instantaneous scaling approximation, c.f. dashed line in fig. 8, the turning-point frequency $f_{\Delta}$ is given by the $(T, f)$-correspondence relation

$$
f_{\Delta}^{\text {scaling }}=\left(4.5 \times 10^{-2} \mathrm{~Hz}\right)\left(\frac{T_{\Delta}}{\mathrm{GeV}}\right)\left(\frac{0.1 \times 50 \times 10^{-11}}{\alpha \Gamma G \mu}\right)^{1 / 2}\left(\frac{g_{*}\left(T_{\Delta}\right)}{g_{*}\left(T_{0}\right)}\right)^{1 / 4} .
$$

However, the deviation from the scaling regime during the change of cosmology, c.f. sec. 4.3, implies a shift to lower frequencies of the $(T, f)$-correspondence, by a factor $\sim 22.5$, c.f. solid vs dashed lines in fig. 8. The correct $(T, f)$-correspondence when applied to a change of cosmology is

$$
f_{\Delta}^{\mathrm{VOS}}=\left(2 \times 10^{-3} \mathrm{~Hz}\right)\left(\frac{T_{\Delta}}{\mathrm{GeV}}\right)\left(\frac{0.1 \times 50 \times 10^{-11}}{\alpha \Gamma G \mu}\right)^{1 / 2}\left(\frac{g_{*}\left(T_{\Delta}\right)}{g_{*}\left(T_{0}\right)}\right)^{1 / 4} .
$$


We fit the numerical factor in eq. (46) (but also in eq. (52)) by imposing ${ }^{3}$ the nonstandard-cosmology spectrum $\Omega_{\mathrm{NS}}$ to deviate from the standard-cosmology one $\Omega_{\mathrm{ST}}$ by $10 \%$ at the turning-point frequency,

$$
\left|\frac{\Omega_{\mathrm{NS}}\left(f_{\Delta}\right)-\Omega_{\mathrm{ST}}\left(f_{\Delta}\right)}{\Omega_{\mathrm{ST}}\left(f_{\Delta}\right)}\right| \simeq 10 \%
$$

We are conservative here. Choosing $1 \%$ instead of $10 \%$ would lead to a frequency shift of the order of $\mathcal{O}(400)$, c.f. eq. (82). Note that our eq. (46) is numerically very similar to the one in [21-23] although an instantaneous change of the loop-production efficiency $C_{\text {eff }}$ at $T_{\Delta}$ is assumed in [21-23]. This can be explained if in Ref. [21-23], the criterion (47) is smaller than the percent level.

\subsection{Constraints}

A long matter/kination era leads to a spectral suppression/enhancement which could lie within the observational windows of future GW observatories. In fig. 9, we show the constraints on an early long-lasting $\left(r \gtrsim 10^{7}\right)$ non-standard era ending at the temperature $T_{\Delta}$, for different values of $G \mu$. We assume that a non-standard era is detectable by a GW interferometer if the absolute value of the observed spectral index is larger than 0.15. This corresponds to the spectral-index prescription ( $R x$ 2) discussed in sec. 9.1. We see that LISA, BBO/DECIGO and ET/CE can probe non-standard eras ending below $T_{\Delta} \simeq 10 \mathrm{TeV}, 100 \mathrm{TeV}$ and $1 \mathrm{TeV}$, respectively. LIGO can already constrain kination eras ending after $10 \mathrm{GeV}$. The temperatures which we can probe are $\mathcal{O}(25)$ larger when assuming a VOS network (c.f. VOS dashed-dotted line in fig. 9) compared to a scaling network (c.f. colored regions in fig. 9), (see sec. 4.3 for the definitions of scaling and VOS networks). Particle production starts to limit the observation for $G \mu \lesssim 10^{-15}$.

\subsection{A shorter period of kination}

Interestingly, a short kination period can generate a bump in the spectrum. We show this in the left panel of fig. 10. In fact, the network has no time to reach the scaling regime. Particularly, on the right panel of fig. 10, we show how the efficiency of the loop production grows with the duration of the kination era, without reaching its scaling value $C_{\text {eff }}=29.6$, c.f. eq. (39). The bump gets higher for longer kination epoch since the network gets closer to its scaling solution. However, this high-frequency feature may not be observable due to the high-frequency cutoff from particle production, c.f. solid purple and red lines in left panel of fig. 10

\footnotetext{
${ }^{3}$ The coefficient in eq. (46) has been fitted upon considering the matter case $\Omega_{\mathrm{NS}}=\Omega_{\mathrm{matter}}$. Note that the turning-point in the kination case is slightly higher frequency by a factor of order 1, c.f. fig. 8 .
} 

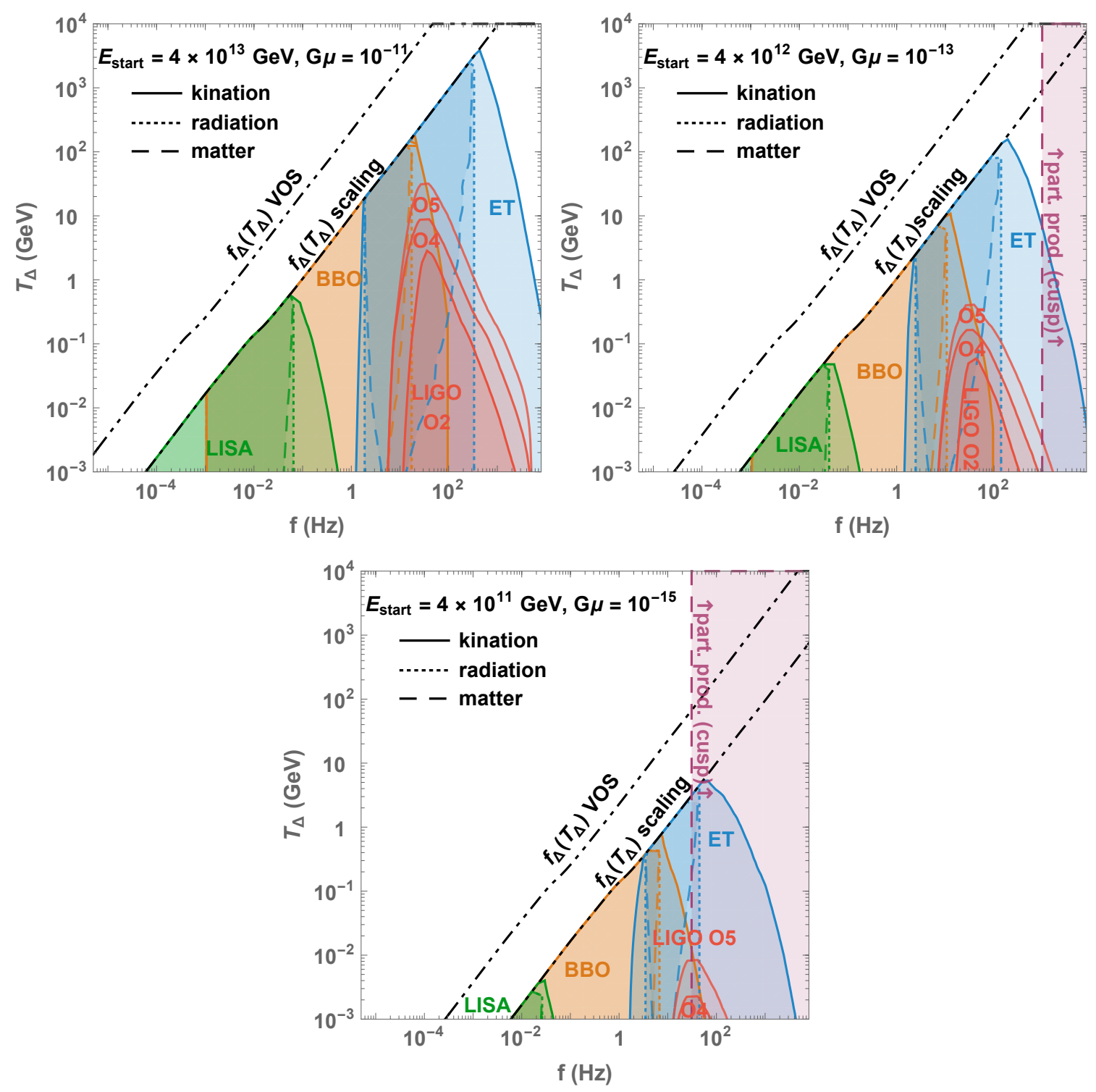

Figure 9: The colored regions show the detectability of a spectral suppression/enhancement, c.f. spectral-index prescription (Rx 2) in sec. 9.1, due to an early matter/kination era taking place before the standard radiation domination. For technical reasons, the instantaneous scaling regime is assumed for the colored regions. We show the reach when going beyond the scaling regime, c.f. sec. 4.3, with a black dashed-dotted line labelled 'VOS'. The limitation from particle production, c.f. sec. 3.4, is considered in purple. 

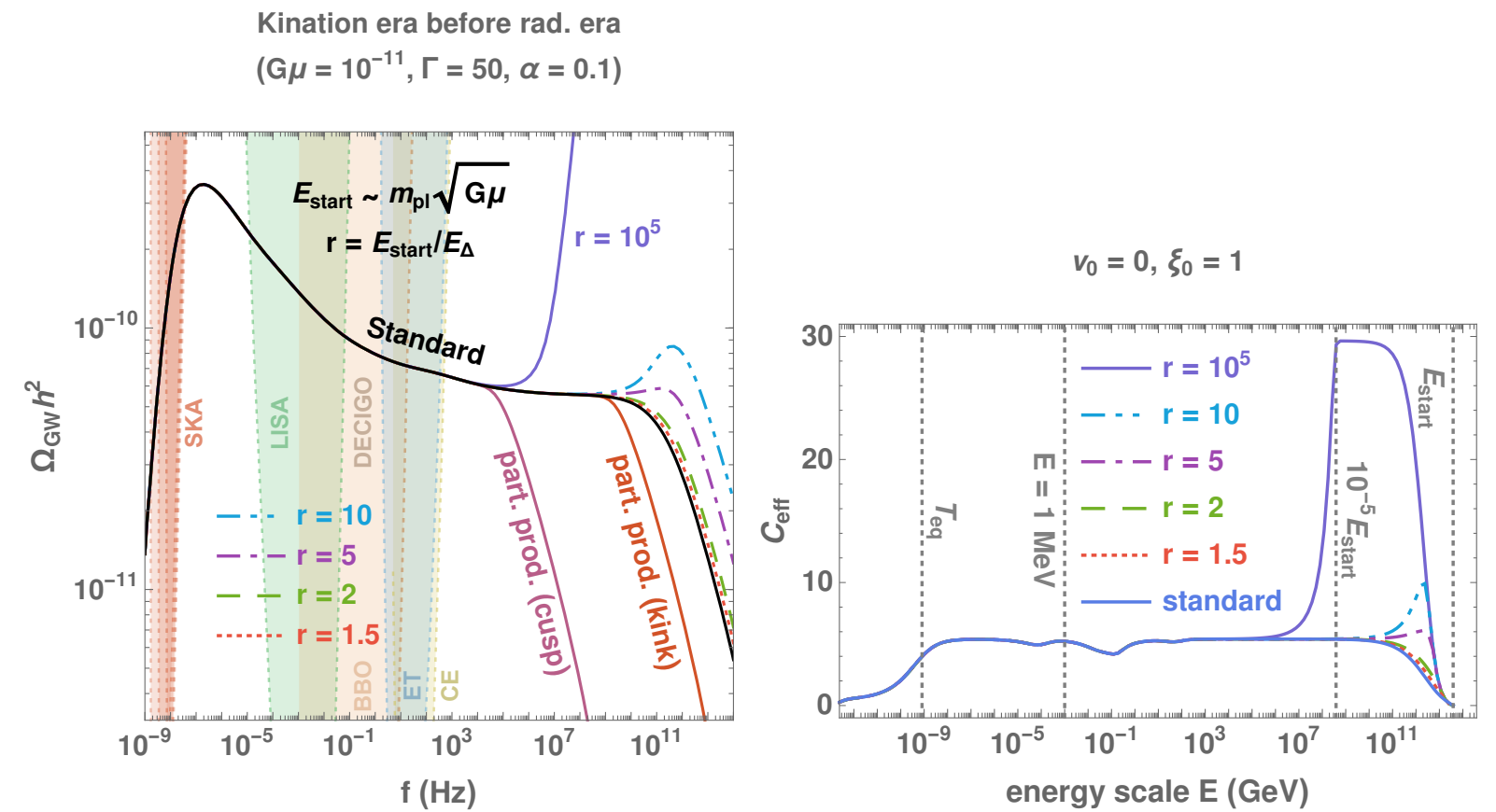

Figure 10: Left: $G W$ spectrum from the string network evolved in the presence of a short kination era after inflation. The peak at high frequencies generated by loops created just after the network formation (see fig. 6) is enhanced by the effect of the kination era. Right: The corresponding evolution of the loop-production efficiency shows that the scaling regime is never reached for short kination eras. 


\section{Intermediate matter era}

\subsection{The non-standard scenario}

In this section, we consider the existence of an early-intermediate-matter-dominated era, following an earlier radiation era and preceeding the standard radiation era. The intermediate matter-dominated era starts when the matter energy density $\rho_{\text {matter }} \propto a^{-3}$ takes over the radiation energy density $\rho_{\text {radiation }} \propto a^{-4}$ and ends when the matter content decays into radiation, c.f. fig. 11. The energy density profile is illustrated in Fig. 11 and can be written as

$$
\rho_{\text {tot }}(a)= \begin{cases}\rho_{\text {rad }}^{\text {st }}(a)+\rho_{\text {late }}(a) & \text { for } \rho>\rho_{\text {start }} \\ \rho_{\text {start }}\left(\frac{a_{\text {start }}}{a}\right)^{n}+\rho_{\text {late }}(a) & \text { for } \rho_{\text {start }}>\rho>\rho_{\text {end }} \\ \rho_{\text {end }} \Delta_{R}\left(T_{\text {end }}, T\right)\left(\frac{a_{\text {end }}}{a}\right)^{4}+\rho_{\text {late }}(a) & \text { for } \rho<\rho_{\text {end }}\end{cases}
$$

where

$$
\begin{aligned}
\rho_{\text {start }}, \rho_{\text {end }} \equiv \text { the starting and ending energy density of the non-standard cosmology, } \\
\rho_{\text {late }} \equiv \text { the standard-cosmology energy density dominating at late times, } \\
\quad \text { e.g. the standard matter density, and cosmological constant. } \\
\Delta_{R} \text { is given in eq.(42). }
\end{aligned}
$$

Intermediate matter era

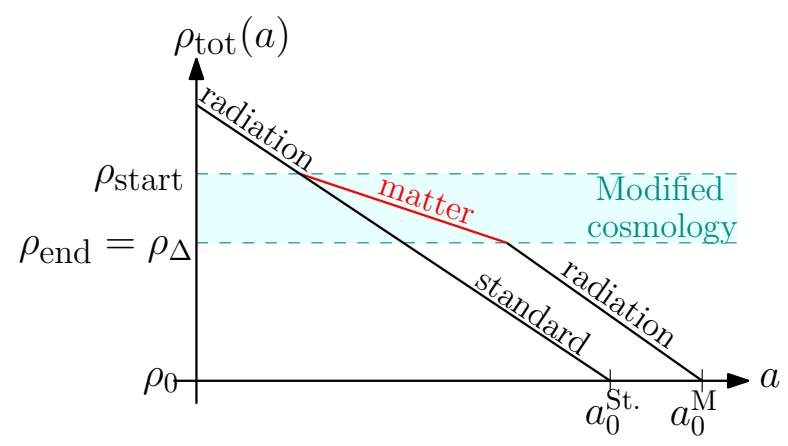

Intermediate matter era

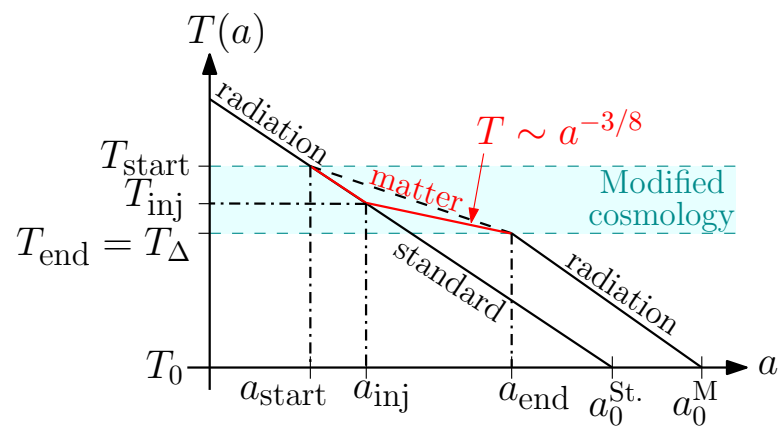

Figure 11: Evolution of the total energy density (left) and the temperature (right) assuming the presence of an intermediate matter era. $T_{\mathrm{inj}}$ and $a_{\mathrm{inj}}$ are the temperature and scale factor at which the entropy injected by the decay of the matter content into radiation, starts to be effective, c.f. fig. 2 in [190]. St: standard; M: matter.

\subsection{Impact on the spectrum: a low-pass filter}

In the left panel of fig. 12, we show that an intermediate matter era blue-tilts the spectral index of the spectrum. Furthermore, at higher frequencies, corresponding to loops produced during the radiation era preceding the matter era, the spectrum recovers a flat scaling but is suppressed by the duration $r$ of the matter era

$$
r=\frac{T_{\text {start }}}{T_{\Delta}}
$$


where $T_{\Delta}=T_{\text {end }}$. The negative spectral index and the suppression can be understood from fig. 11. Indeed, the universe, in the presence of an intermediate matter era, has expanded more than the standard universe. Hence at a fixed emitted frequency, loops are produced later and so are less numerous, implying less GW emission. In the right panel of fig. 12, we show that for short intermediate matter era, $r=2$ or $r=10$, the scaling regime in the matter era, which is characterized by $C_{\text {eff }}=0.39$, c.f. eq. 39 , is not reached.

$$
\begin{gathered}
\text { Intermediate matter } \\
\left(G \mu=10^{-11}, \Gamma=50, \alpha=0.1\right)
\end{gathered}
$$
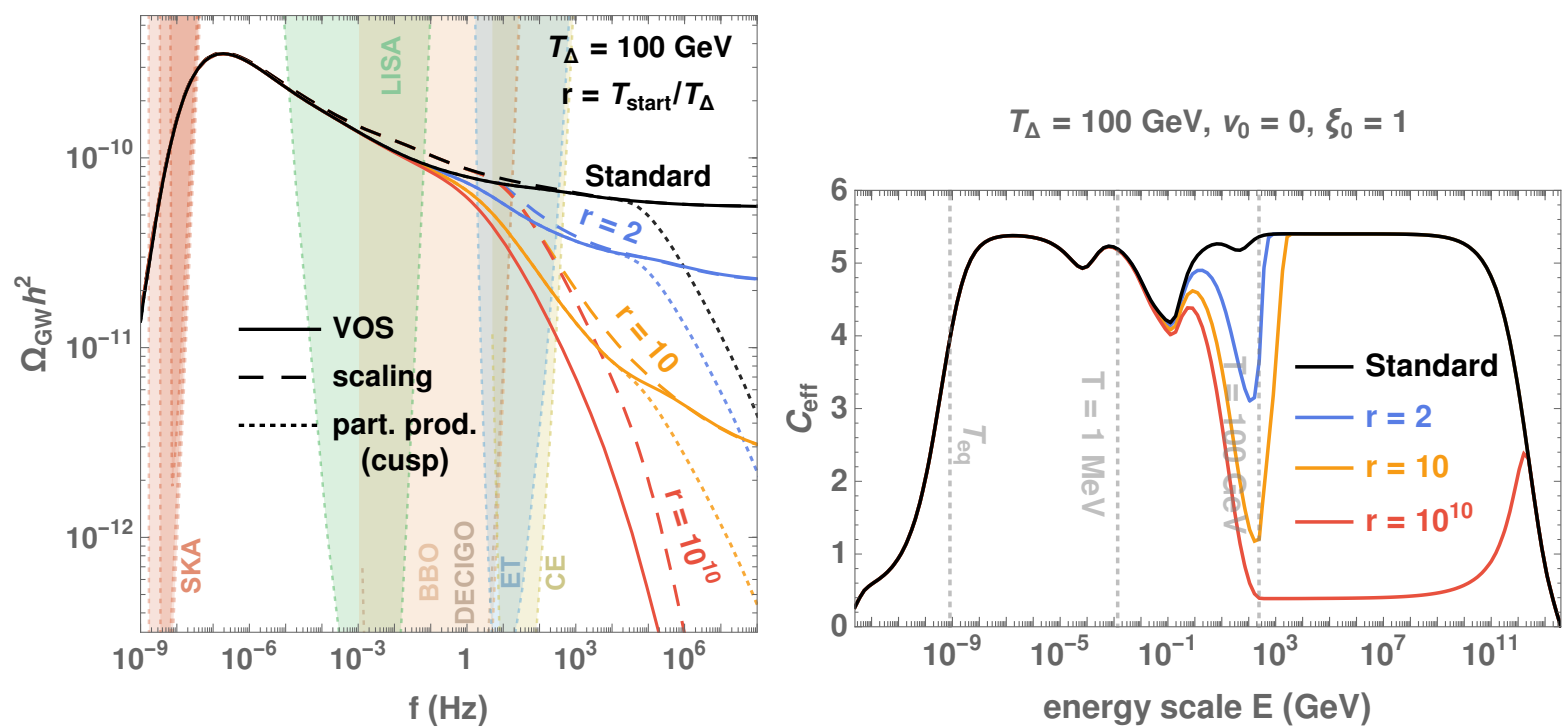

Figure 12: $\quad G W$ spectrum from an intermediate matter era starting at the temperature $T_{\text {start }}$ and ending at $T_{\Delta}$. Left: The dashed-lines assume that the scaling regime in matter era switches instantaneously to the scaling regime in radiation era, meaning that $C_{\mathrm{eff}}$ varies discontinuously, whereas the plain lines incorporate the transient behavior solution of the VOS equations and shown on the right panel. Right: Time evolution of the loop-production efficiency $C_{\text {eff }}$ after solving the VOS equations, c.f. sec. 4.3.

\subsection{Constraints}

In fig. 13, we show the constraints on the presence of an early-intermediate-non-standardmatter-dominated era starting at the temperature $r T_{\Delta}$ and ending at the temperature $T_{\Delta}$. Matter eras as short as $r=2$ and ending at temperature as large as $100 \mathrm{TeV}$ could be probed by GW interferometers. We assume that an early-matter era is detectable if the spectral index is smaller than -0.15 , c.f. spectral-index prescription (Rx 2) in sec. 9. In a companion paper [53], we provide model-independent constraints on the abundance and lifetime of an unstable particles giving rise to such a non-standard intermediate matter era. 

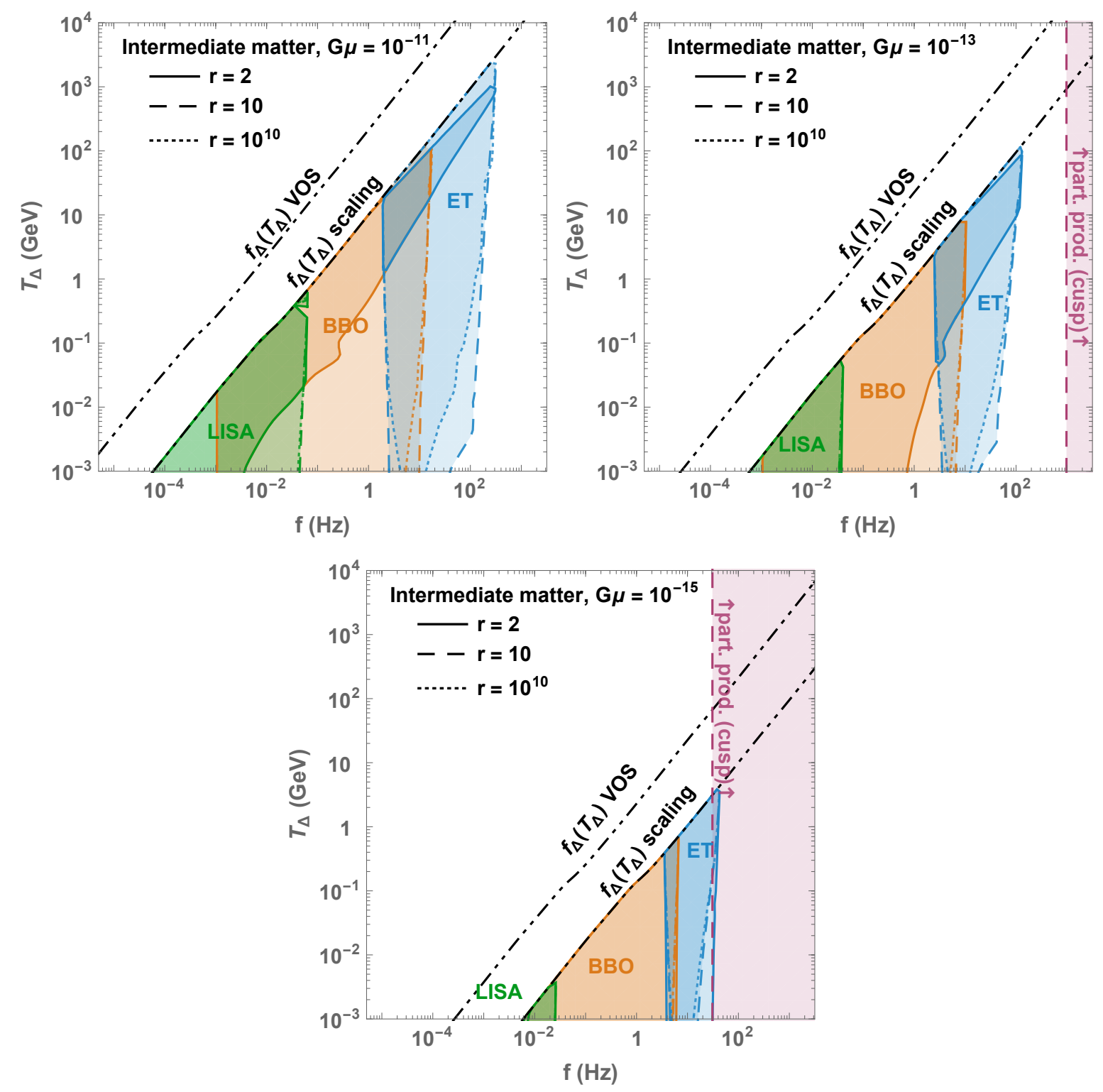

Figure 13: The colored regions show the detectability of the spectral suppression, c.f. spectralindex prescription (Rx 2) in sec. 9.1, due to a NS intermediate matter era with duration $r=$ $T_{\text {start }} / T_{\Delta}$. For technical reasons, in the colored region the scaling cosmic-string network is assumed but the reach of the VOS model, c.f. sec. 4.3, is given by a black-dashed-dotted line labelled 'VOS'. Limitation from particle production, c.f. sec. 3.4, is shown in purple.

\section{Intermediate inflationary era}

\subsection{The non-standard scenario}

Next, we consider the existence of a short inflationary period with a number of e-folds

$$
N_{e} \equiv \log \left(\frac{a_{\text {start }}}{a_{\text {end }}}\right)
$$

smaller than $N_{e} \lesssim 20 \ll 60$, in order not to alter the predictions from the first inflation era regarding the $\mathrm{CMB}$ power spectrum. On the particle physics side, such a short inflationary period can be generated by a highly supercooled first-order phase transition. 
It was stressed that nearly-conformal scalar potentials naturally lead to such short, with $N_{e} \sim 1-15$, periods of inflation [46, 47, 49]. Those are well-motivated in new strongly interacting composite sectors arising at the $\mathrm{TeV}$ scale, as invoked to address the Higgs hierarchy problem and were first studied in a holographic approach [43, 44] (see also the review [191]). As the results on the scaling of the bounce action for tunnelling and on the dynamics of the phase transitions do essentially not depend on the absolute energy scale, but only on the shallow shape of the scalar potential describing the phase transition, those studies can thus be extended to a large class of confining phase transitions arising at any scale. In this section, we will take this inflationary scale as a free parameter.

We define the energy density profile as, c.f. figure 14

$$
\rho_{\mathrm{tot}}(a)= \begin{cases}\rho_{\mathrm{rad}}^{\mathrm{st}}(a)+\rho_{\mathrm{late}}(a) & \text { for } \rho>\rho_{\mathrm{inf}}, \\ \rho_{\mathrm{inf}}=E_{\mathrm{inf}}^{4} & \text { for } \rho=\rho_{\mathrm{inf}} \\ \rho_{\mathrm{inf}} \Delta_{R}\left(T_{\mathrm{end}}, T\right)\left(\frac{a_{\mathrm{end}}}{a}\right)^{4}+\rho_{\mathrm{late}}(a) & \text { for } \rho<\rho_{\mathrm{inf}}\end{cases}
$$

where $\rho_{\text {inf }}$ is the total energy density of the universe during inflation and $E_{\text {inf }} \equiv \rho_{\text {inf }}^{1 / 4}$ is the corresponding energy scale. The function $\Delta_{R}$ is defined in (42).

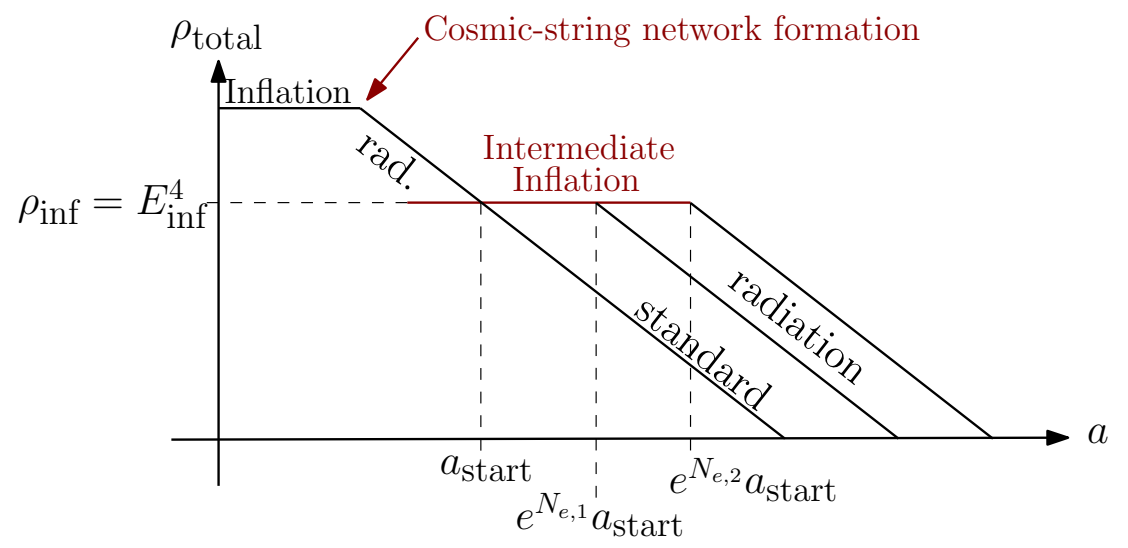

Figure 14: Evolution of the total energy density assuming the presence of an intermediate inflationary era characterised by the energy density $\rho_{\text {inf }}$, for two different durations (number of efolds), $N_{e, 1}$ and $N_{e, 2}$.

\subsection{The stretching regime and its impact on the spectrum}

Fig. 16 shows how the fast expansion during inflation suppresses the GW spectrum for frequencies above a turning-point frequency $f_{\Delta}$ which depends on the number of e-folds. The larger the number of e-folds, the lower $f_{\Delta}$. Indeed, during inflation, the loop-production efficiency $C_{\text {eff }} \propto \xi^{-3}$ is severely suppressed, c.f. fig. 16, by the stretching of the correlation length $\xi$ beyond the Hubble horizon, and loop production freezes [24]. After the end of inflation, one must wait for the correlation length to re-enter the horizon in order to reach the scaling regime again. The duration of the transient regime receives an enhancement factor $\exp N_{e}$. As a result, the turning-point frequency $f_{\Delta}$ receives a suppression factor $\exp N_{e}$ as derived below:

$$
f_{\Delta}=\left(1.5 \times 10^{-4} \mathrm{~Hz}\right)\left(\frac{T_{\mathrm{re}}}{\mathrm{GeV}}\right)\left(\frac{0.1 \times 50 \times 10^{-11}}{\alpha \Gamma G \mu}\right)^{1 / 2}\left(\frac{g_{*}\left(T_{\mathrm{re}}\right)}{g_{*}\left(T_{0}\right)}\right)^{1 / 4},
$$




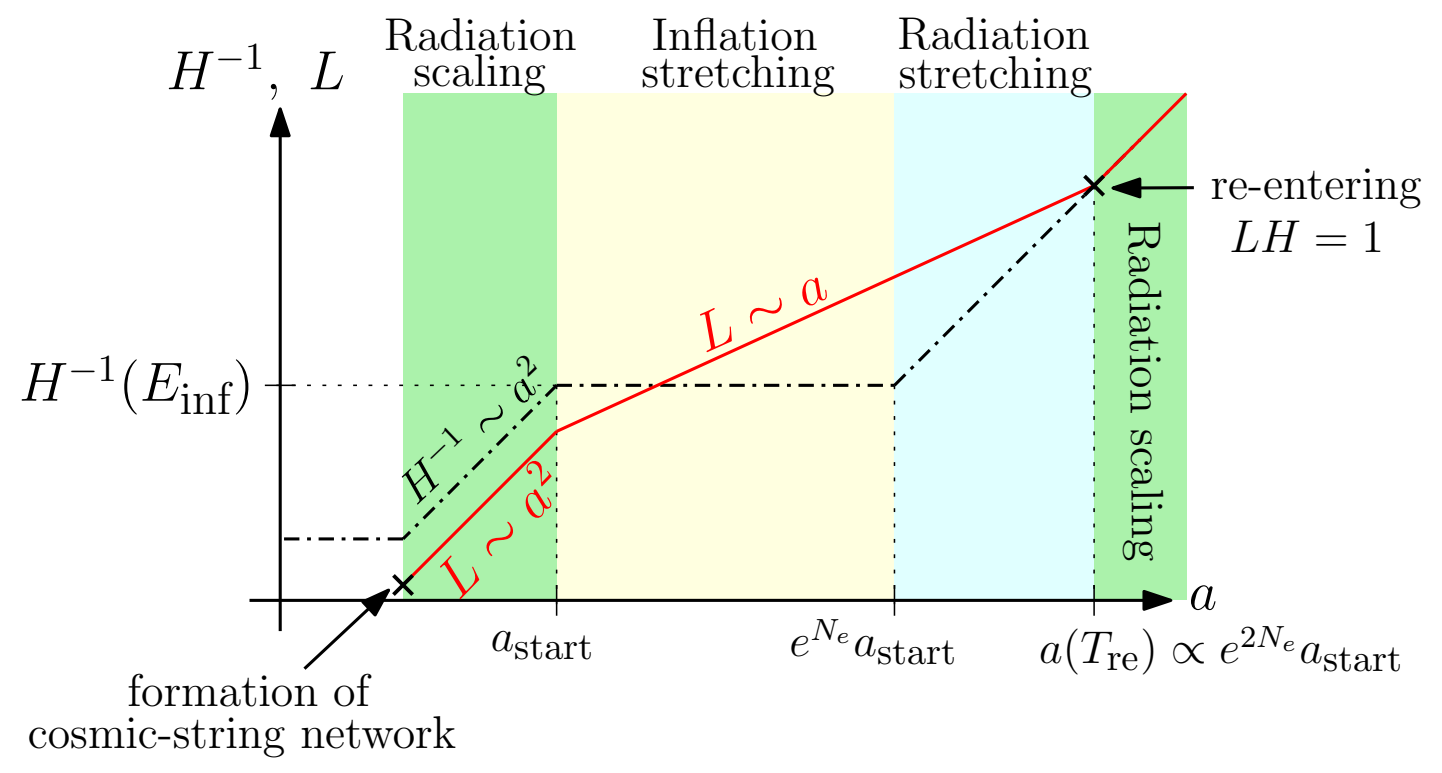

Figure 15: After its formation, before inflation, the network enters the scaling regime with $L \sim a^{2}$ due to loop formation. During the $N_{e}$ e-folds of inflation, the network correlation length gets stretched out of the horizon by the rapid expansion and loop formation stops, thus $L \sim a$. After inflation, during radiation, the correlation length starts to re-enter the horizon and scales again as $L \sim a^{2}$.

with $T_{\mathrm{re}}$ the temperature at which the long-string network re-enters the Hubble horizon

$$
T_{\mathrm{re}} \simeq \frac{E_{\mathrm{inf}}}{(0.1) g_{*}^{1 / 4}\left(T_{\mathrm{re}}\right) \exp \left(N_{e}\right)},
$$

where $(0.1)$ is the typical correlation length before the stretching starts. Note that the numerical factor in (52) comes from the demanded precision of $10 \%$ deviation, c.f. eq. (47). It can be lower by a factor $\sim 300$ if the $1 \%$ precision is applied, as shown in eq. (83).

Fig. 17 shows how a sufficiently long period of intermediate inflation can lead to SGWB with peak shapes in the future GW interferometer bands. We emphasize that the change of the GW spectrum from CS in presence of a non-standard matter-dominated era, a short inflation, and particle production look similar. Therefore, the question of how disentangling each effect from one another deserves further studies.

Derivation of the turning-point formula (inflation case): Let us review the chronology of the network in the presence of an intermediate-inflation period (see fig. 15) in order to derive eq. (52). In the early radiation era, the network has already been produced and reached the scaling regime before inflation starts. The correlation length scale is of order $(0.1) t$ or equivalently

$$
L_{\text {start }} H_{\text {start }} \sim \mathcal{O}(0.1),
$$

where $L$ is the correlation length of strings, and $H$ is the Hubble rate. When inflation begins, it stretches cosmic strings beyond the horizon with

$$
L \propto a \quad \text { leading to } L H \gg 1,
$$


Intermediate Inflation: $E_{\text {inf }}=100 \mathrm{TeV}$

$$
\left(\mathrm{G} \mu=10^{-11}, \Gamma=50, \alpha=0.1\right)
$$

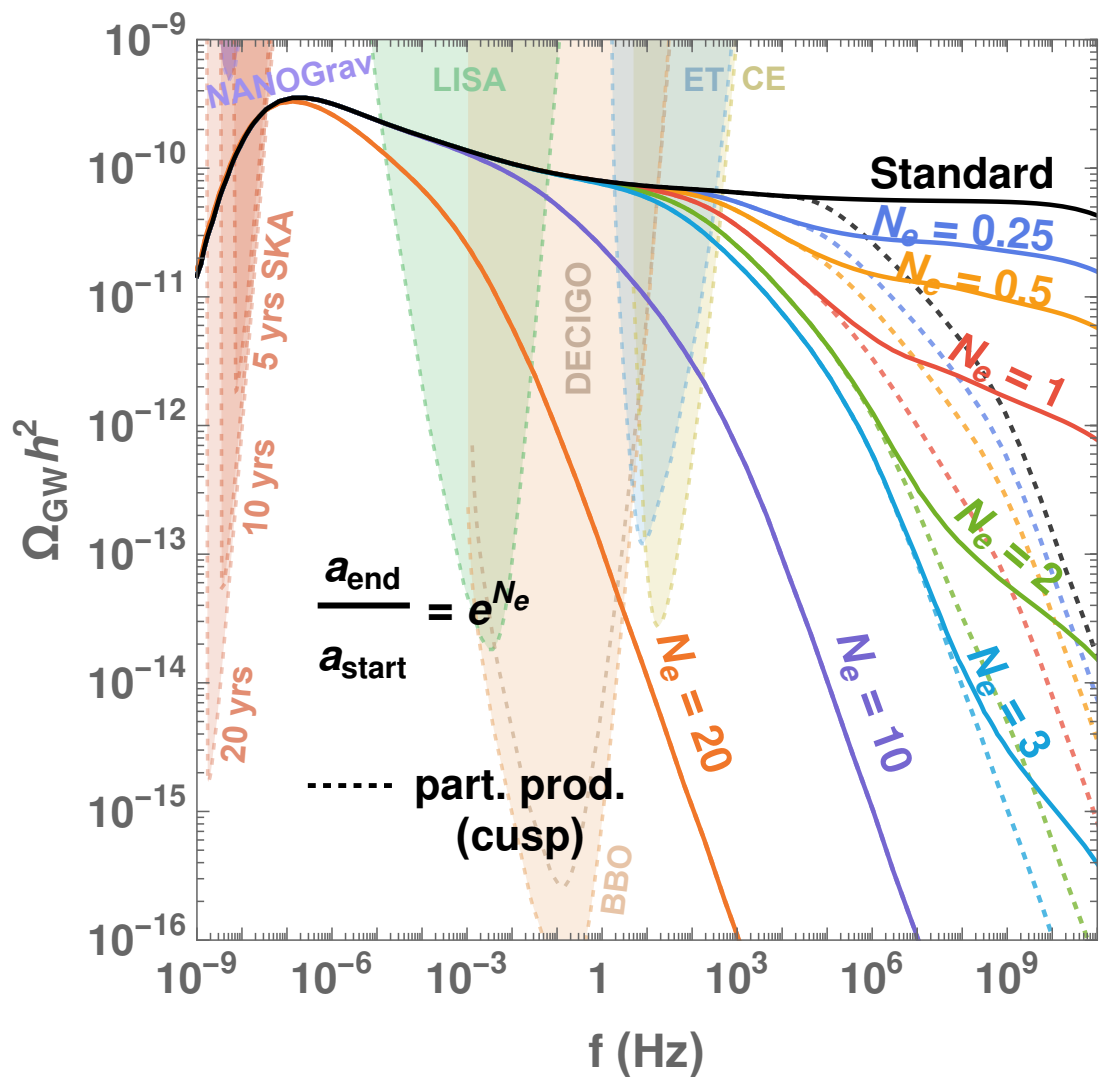

$\left(v_{0}=0, \xi_{0}=1\right)$
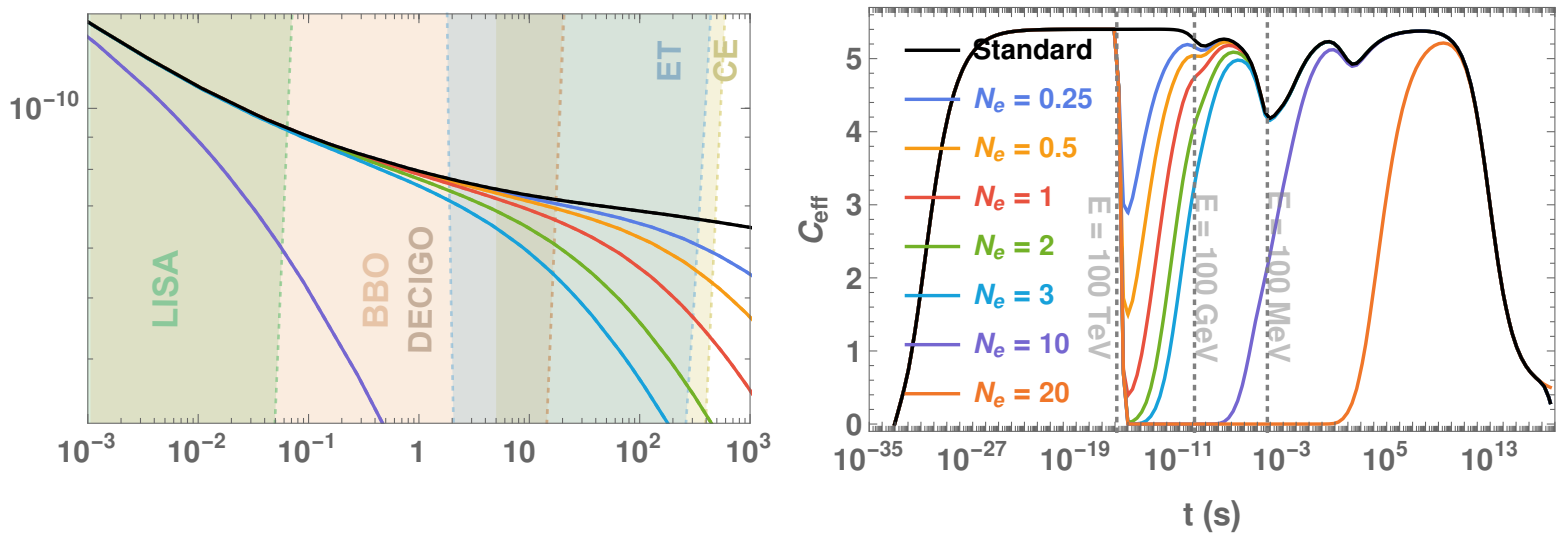

Figure 16: Top: GW spectra from cosmic strings assuming either the scaling or the VOS network, evolved in the presence of a non-standard intermediate inflation ear. Inflation directly affects the VOS parameters by stretching the strings beyond the horizon. Bottom: The loopproduction is suppressed and only becomes significant again when the correlation length re-enters the horizon. Limitations due to particle production, c.f. sec. 3.4, are shown with dotted lines.

within a few e-folds. Later, the late-time energy density takes over inflation, but the network is still in the stretching regime $L \propto a$, i.e.

$$
L H \propto t^{(2-n) / n} \quad \text { during the era with } \rho \propto a^{n} .
$$




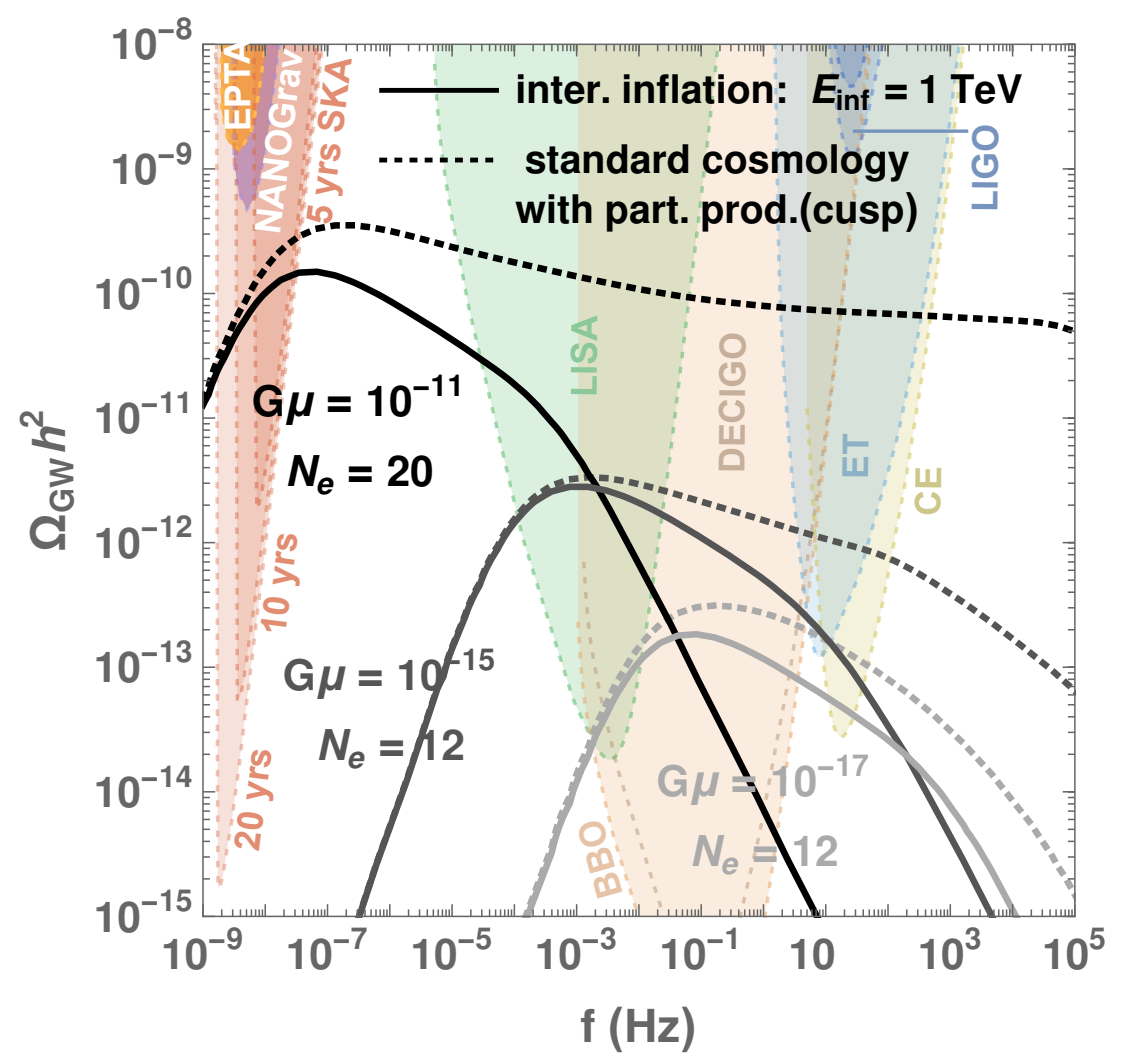

Figure 17: In the case an intermediate inflationary era lasting for $\mathcal{O}(10)$ efolds, the $S G W B$ from cosmic strings completely looses its scale invariant shape and has instead a peak structure. A TeV scale inflation era can lead to broad peaks either in the LISA or BBO band or even close to the SKA band, depending on the value of the string tensions $G \mu$, and the number of efolds $N_{e}$.

For $n>2$, the Hubble horizon will eventually catch up with the string length, allowing them to re-enter, and initiate the loop production. We consider the case where the universe is radiation-dominated after the inflation period and define the temperature $T_{\text {re }}$ of the universe when the long-string correlation length $L$ re-enters the horizon

$$
L_{\mathrm{re}} H_{\mathrm{re}}=1
$$

where $L_{\mathrm{re}}$ and $H_{\mathrm{re}}$ are the correlation length and Hubble rate at the re-entering time. We can use eq. (56) to evolve the correlation length, starting from the start of inflation up to the re-entering time

$$
\begin{aligned}
1=L_{\mathrm{re}} H_{\mathrm{re}} & =\left(\frac{t_{\mathrm{re}}}{t_{\mathrm{end}}}\right)^{-1 / 2} L_{\mathrm{end}} H_{\mathrm{end}}, \\
& =\left(\frac{t_{\mathrm{re}}}{t_{\mathrm{end}}}\right)^{-1 / 2}\left(\frac{a_{\mathrm{end}}}{a_{\text {start }}}\right) L_{\text {start }} H_{\text {start }}, \\
& \simeq\left(\frac{T_{\mathrm{re}}}{T_{\mathrm{end}}}\right) e^{N_{e}}(0.1)
\end{aligned}
$$

We have used $t \propto T^{-2}$ during the radiation era and introduced the number $N_{e}$ of inflation e-folds. Finally, we obtain the re-entering temperature in terms of the number of e-folds 
$N_{e}$ and the inflationary energy scale $E_{\text {inf }}$ as

$$
T_{\mathrm{re}} \simeq \frac{E_{\mathrm{inf}}}{(0.1) g_{*}^{1 / 4}\left(T_{\mathrm{re}}\right) \exp \left(N_{e}\right)} .
$$

After plugging eq. (61) into the VOS turning-point formula (46), with $T_{\Delta}=T_{\text {re }}$, and adjusting the numerical factor with the GW spectrum computed numerically, we obtain the relation in eq. (52) between the turning-point frequency and the inflation parameters $N_{e}$ and $E_{\text {inf }}$.
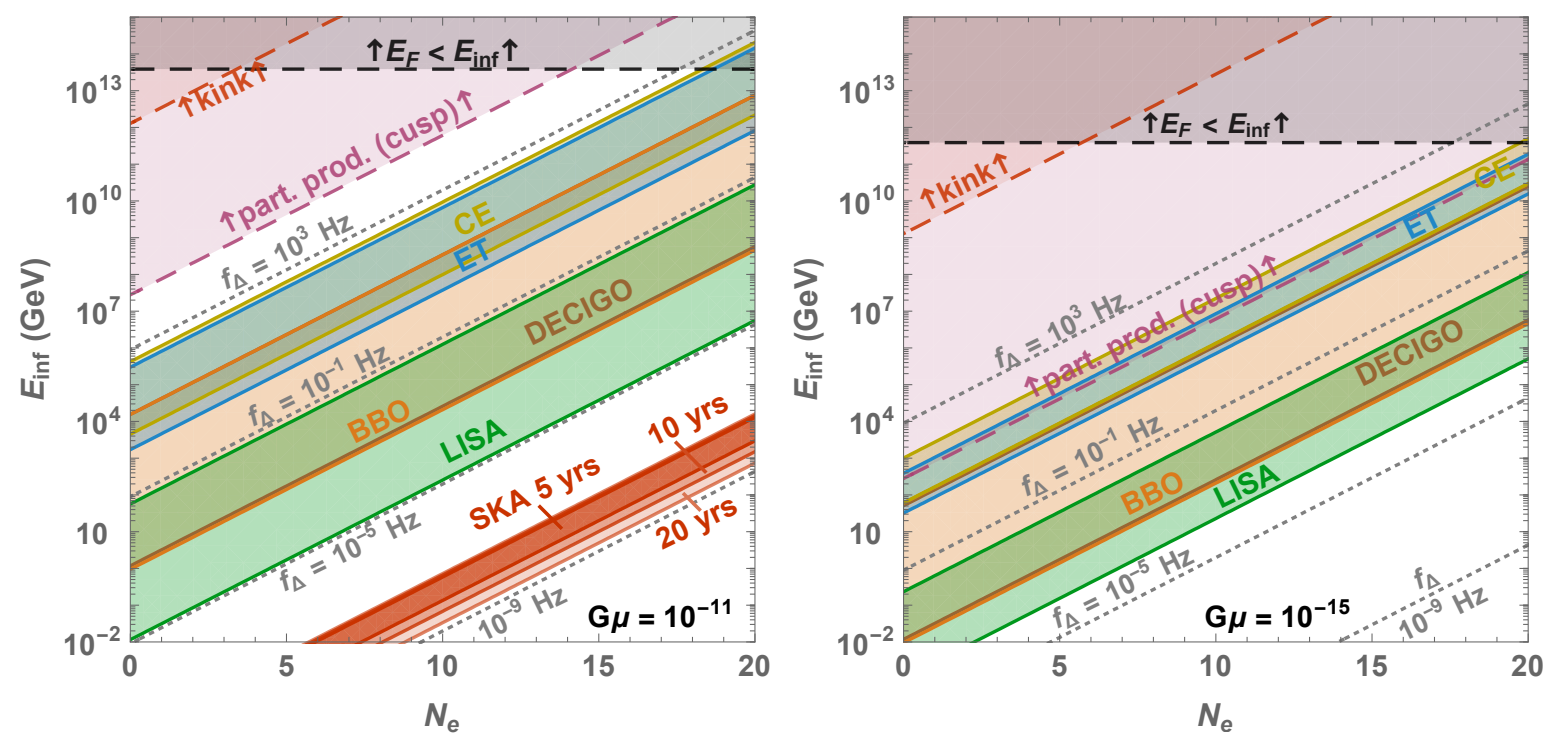

Figure 18: Reach of future $G W$ interferometers for probing an intermediate-inflation period with an energy scale $E_{\text {inf }}$, lasting $N_{e}$ efolds. Colored regions correspond to the turning-points with amplitude higher than each power-law-sensitivity curve, c.f. turning-point prescription (Rx 1) in sec. 9.1. Gray dotted lines are turning-points, c.f. eq. (52), for given frequencies. Red and purple dashed lines are limitations from particle production, c.f. sec. 3.4.

\subsection{Constraints}

In fig. 18, 19 and 20, we show the constraints on an intermediate short inflation period in the planes $E_{\mathrm{inf}}-N_{e}, G \mu-E_{\mathrm{inf}}$, and $G \mu-N_{e}$, respectively. We follow the turningpoint prescription ( $R x$ 1) defined in sec. 9 , which constrains a non-standard cosmology by using the detectability of the turning-point frequency defined by eq. (52). The longer the intermediate inflation, the later the correlation length re-enters the horizon, the latter the long-string network goes back to the scaling regime, the lower the frequency of the turning-point and the larger the inflationary scale which can be probed. The detection of a GW spectrum generated by CS by future GW observatories would allow to probe an inflationary energy scale $E_{\text {inf }}$ between $10^{-2} \mathrm{GeV}$ and $10^{13} \mathrm{GeV}$ assuming a number of e-folds $N_{e} \lesssim 20$. 

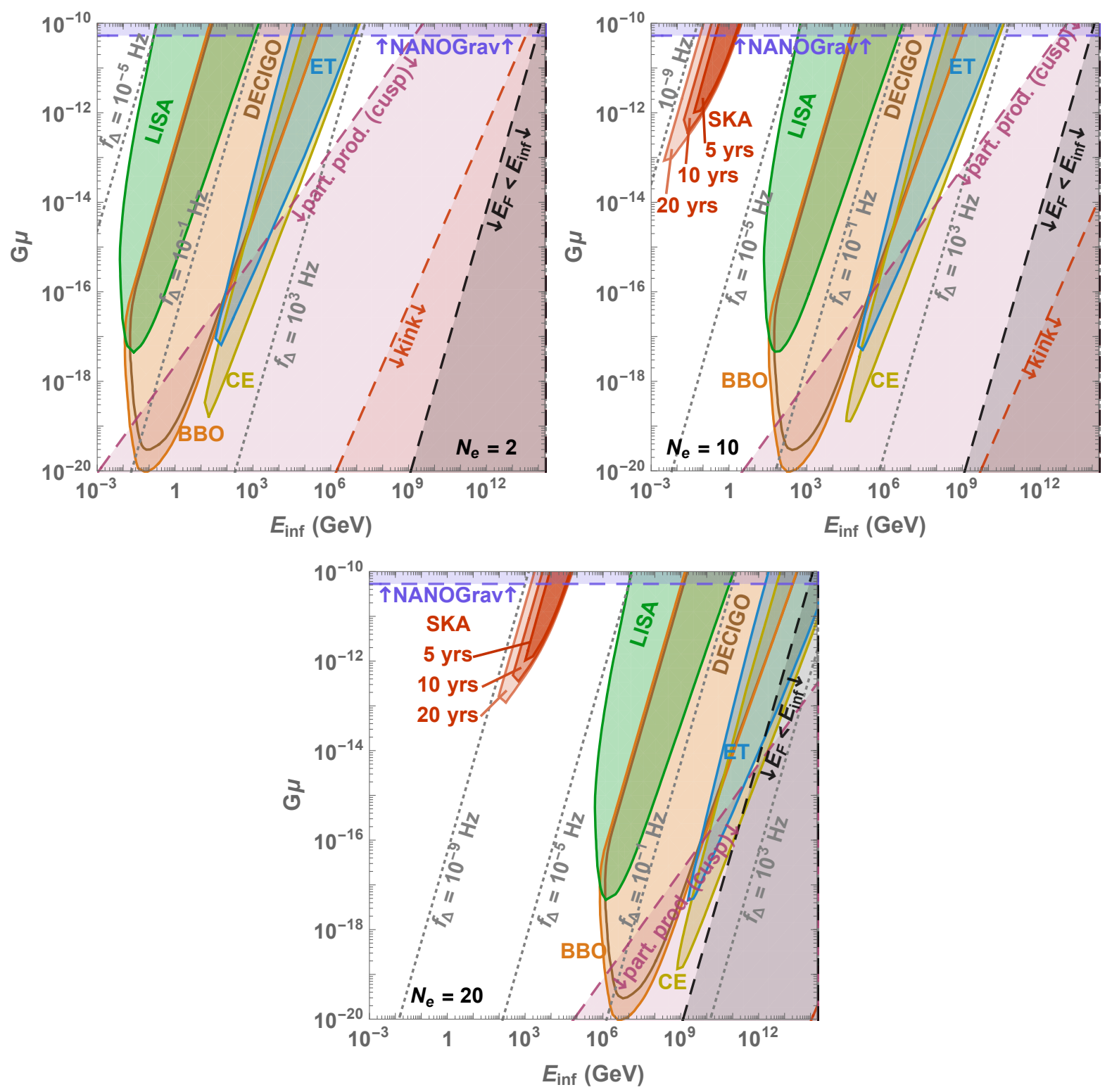

Figure 19: Constraints on intermediate inflation from $C S$ detection by future $G W$ observatories. The longer the intermediate inflation, the later the correlation length re-enters the horizon, the more shifted to lower frequencies the turning-point and the larger the inflation scale which we can probe. The bound $E_{F}<E_{\text {inf }}$, where $E_{F} \sim m_{p l} \sqrt{G \mu}$ is the network-formation energy scale, guarantees that the CS network forms before the intermediate-inflation starts. Red and purple dashed lines are limitations from particle production, c.f. sec. 3.4.

\section{$9 \quad$ Detectability of spectral features}

\subsection{Two prescriptions}

We aim at using the would-be detection of a SGWB spectrum generated by CS to constrain an early non-standard era. We assume the detection of a SGWB from CS by a detector $(i)$ with sensitivity $\Omega_{\text {sens }}^{(i)}(f)$

$$
\Omega_{\mathrm{sens}}^{(i)}(f) \gtrsim \Omega_{\mathrm{GW}}(f) .
$$



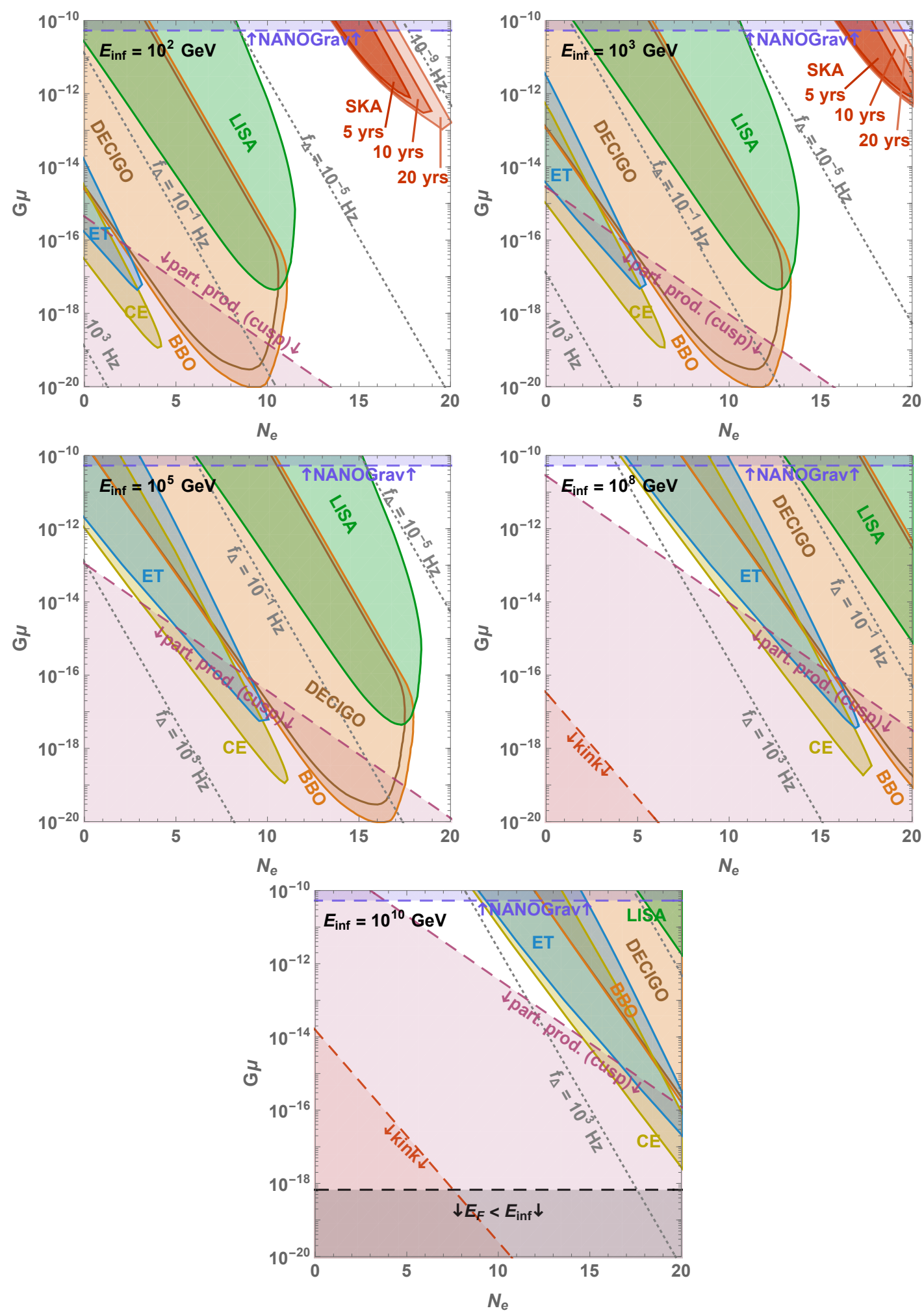

Figure 20: Prospect constraints on intermediate inflation if a $G W$ interferometer detects a $S G W B$ from $C S$ with tension $G \mu$. The freezing of the long-string network due to the stretching of the correlation length outside the horizon allows to probe large inflationary scale $E_{\text {inf for large }}$ number of efolds $N_{e}$. Red and purple dashed lines are limitations from particle production, c.f. sec. 3.4. 

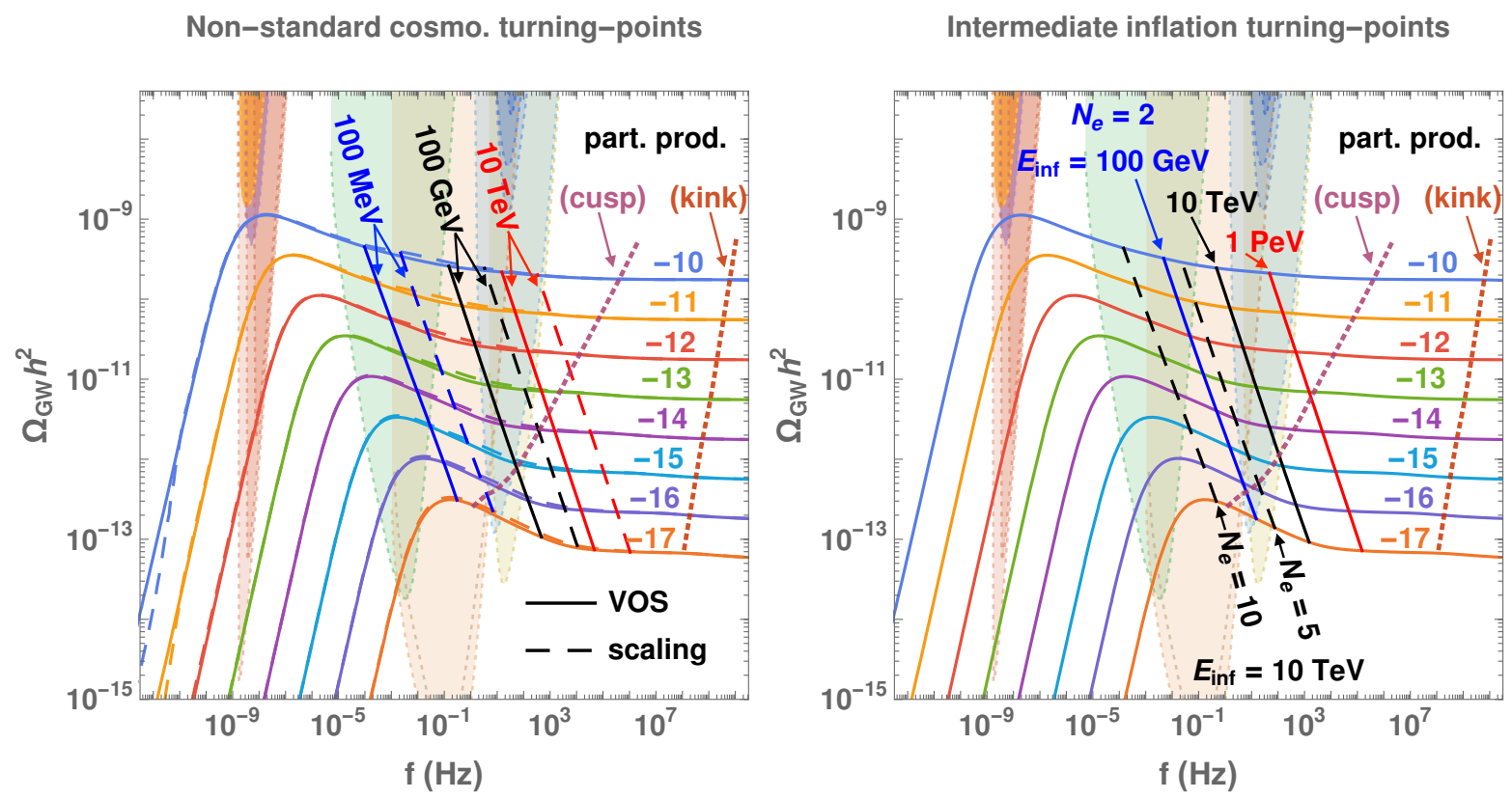

Figure 21: Left: Straight solid and dashed lines are a collection of VOS and scaling turning

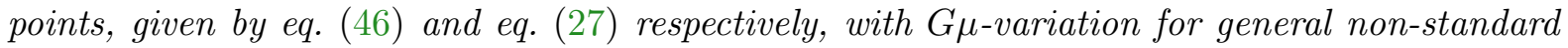
cosmologies ending at temperature $T_{\Delta}$. The displayed spectra assumes a standard cosmology.

Right: We show the turning-points, given by eq. (52), for intermediate inflation lasting for $N_{e}$ e-folds and taking place at the energy scale $E_{\text {inf. }}$. The dotted lines in the two panels show the cut-off frequencies due to particle productions, c.f. sec. 3.4, for each value of G $\mu$.

The power-law integrated sensitivity (PLS) curves of the different experiments are computed in app. F.kination We propose two prescriptions for detecting a non-standard era $\left(T_{\Delta}, r\right)$ :

- $\mathbf{R x} \mathbf{1}$ (turning-point prescription): The frequency $f_{\Delta}$ of the turning-point where the standard and non-standard spectra meet must be inside the interferometer window. GW detected at frequency $f_{\Delta}$ have been emitted by loops formed during the change of cosmology at the temperature $T_{\Delta}$. The relation between $f_{\Delta}$ and $T_{\Delta}$ is given in eq. (45), (46) for a non-inflationary non-standard era, or eq. (52) for an intermediate inflation era, using the $10 \%$ prescription (see eq. (82) and (83) for other cases).

- $\mathbf{R x} 2$ (spectral-index prescription): The absolute value of the observed spectral index $\beta$, which is defined as $\Omega_{\mathrm{GW}}(f) \propto f^{\beta}$, is larger than 0.15 .

The second prescription assumes that the non-standard era tilts the spectral index by more than a benchmark value. We checked that the choice of the precise benchmark value, e.g. 0.15 , has very little impact on the results.

\subsection{More details on the turning-point prescription}

Turning points of non-inflationary-non-standard-eras, c.f. eq. (46), are plotted in the left panel of fig. 21, for different values of $G \mu$ and temperatures $T_{\Delta}$ at the end of the non-standard era. We show the shift to lower frequencies by a factor $\sim 22.5$ due to the deviation from the scaling regime during the change of cosmology.

Turning points in the special intermediate-inflation-era scenario, c.f. eq. (52), are plotted in the right panel of fig. 21, for different inflation scales $E_{\text {inf }}$ and e-fold numbers 

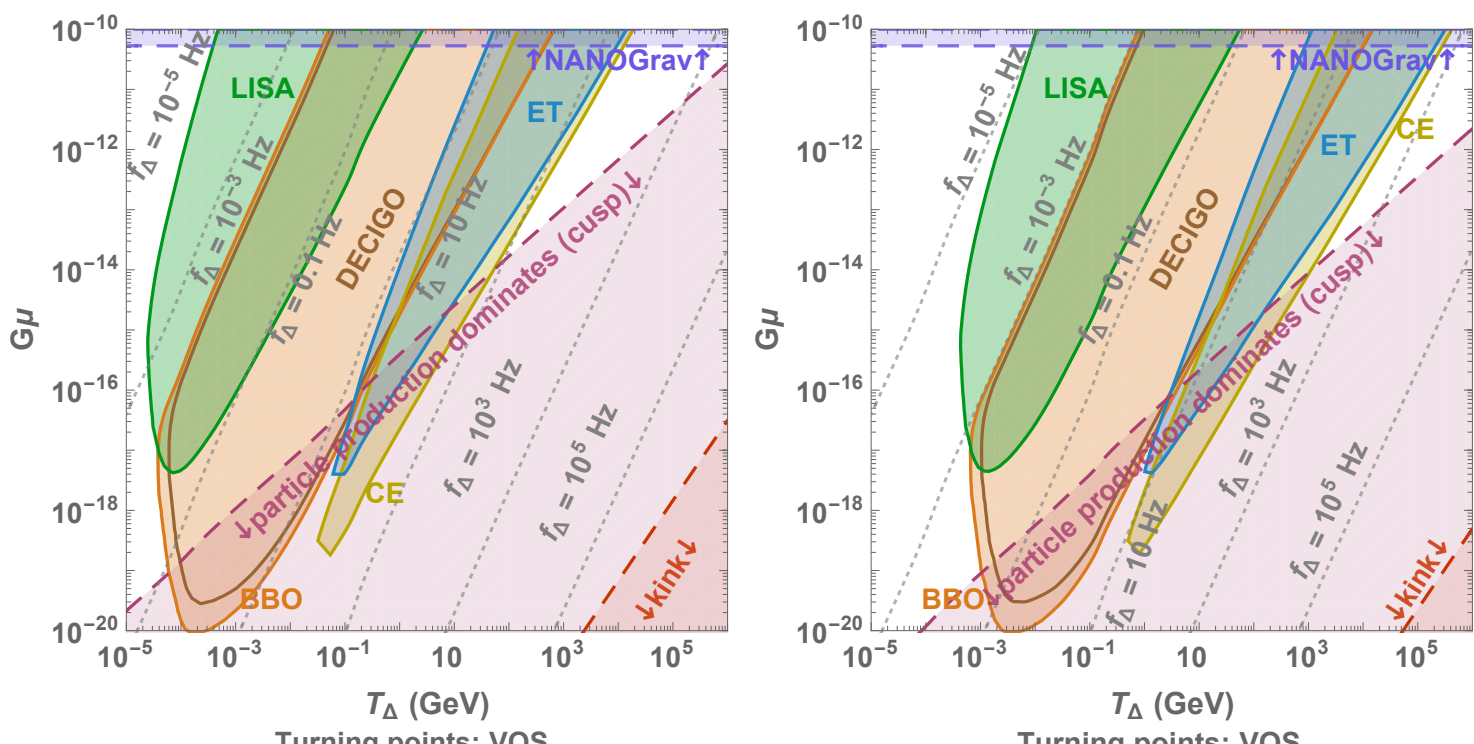

Turning points: vOS
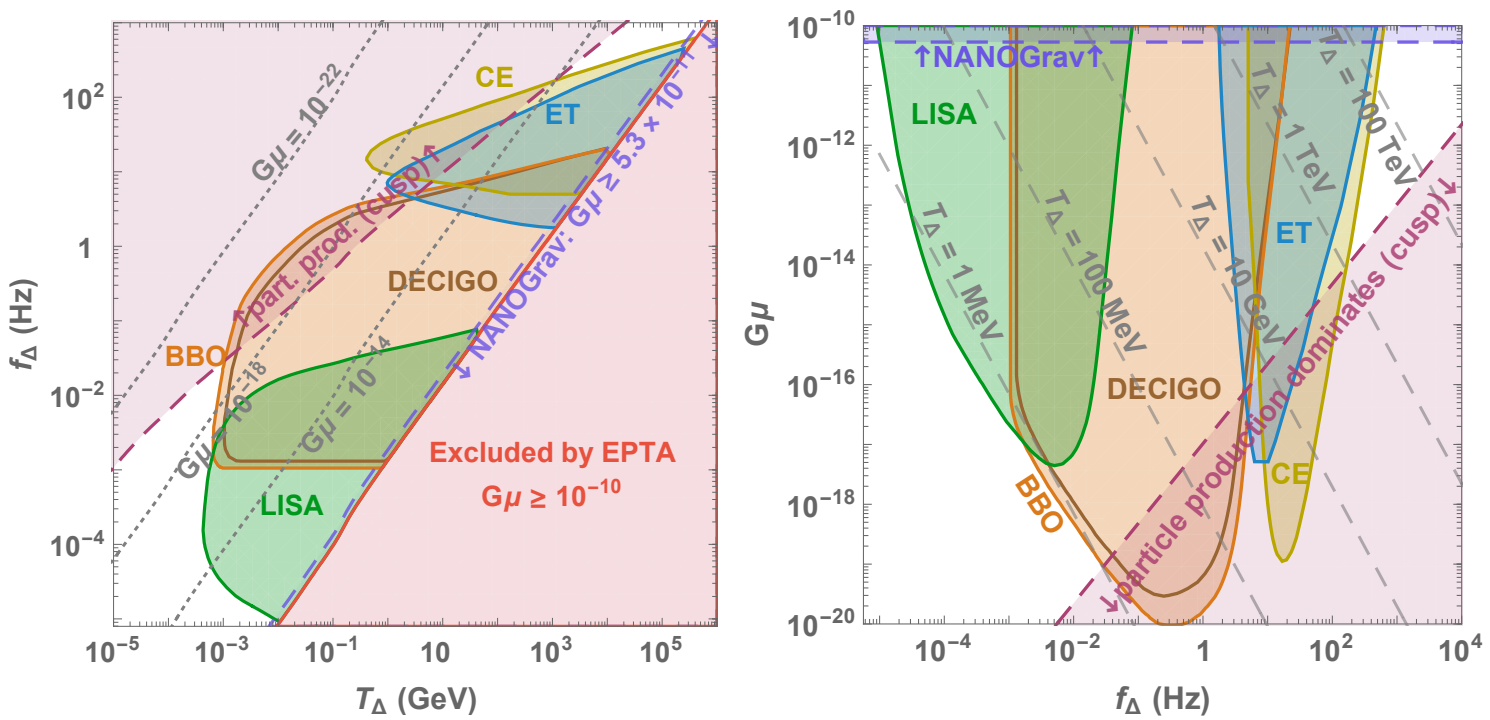

Figure 22: Top: Comparison between the detectability of the turning-points in $G \mu-T_{\Delta}$ planes assuming a scaling network (left) with the one assuming the full VOS evolution (right), c.f. sec. 4.3. Gray dotted lines are turning-points for given frequencies, c.f. eq. (27) for scaling netwok and eq. (46) for VOS network. Bottom: Detectability of turning points in the planes $f_{\Delta}-T_{\Delta}$ and $G \mu-f_{\Delta}$ assuming a VOS network. Limitations from particle production (see sec. 3.4) and bounds from EPTA are also included.

$N_{\mathrm{e}}$. Due to the stretching of the correlation length outside the horizon and the necessity to wait that it re-enters in order to reach the scaling regime, the longer the inflation the lower the turning-point frequency.

With the solid purple and red lines, we show the expected cut-off frequencies above which the GW spectrum is expected to be suppressed due to the domination of massive particle production over gravitational emission, in the benchmark cases where the loop small-scale structures are dominated either by cusps or kinks. Hereby, we show the possibility of losing the information about the cosmological evolution when the turning- 
points are at higher frequencies than the particle-production cut-off.

In fig. 22 , we show the detectability of a turning point at frequency $f_{\Delta}$, corresponding to a change of cosmology taking place at the temperature $T_{\Delta}$, in the plane $G \mu-T_{\Delta}$, $f_{\Delta}-T_{\Delta}$, and $G \mu-f_{\Delta}$. We compare the turning-point formula, defined in eq. 46 in the VOS regime with the one defined in eq (27) in the scaling regime.

Some of these plots were already presented in [21, 22] (for long matter and kination era), assuming that the scaling regime holds during the change of cosmology. Our plots turn out to be similar due to their different choice of precision in the determination of the turning point frequency, see criterion in eq. (47).

\subsection{Comparative reach of different observatories at a glance}

Our analysis shows the complementarity between distinct experiments to probe different energy scales and durations of the non-standard era, as well as different string tensions. As our contour plots in Fig. 9, 13, 18, 19, 20, 22 show, it is not really possible to associate a given new physics energy scale to a given frequency band of observation. In fact, a given frequency band can probe different energy scales, depending on the nature and duration of the non-standard era, and the value of the string tension. Still, we provide some overall comparison in Fig. 23 of the reach of each experiment on the energy scale of the non-standard era. The precise numbers depend on the definition of the observable used to probe the non-standard physics. For any non-inflationary non-standard era (upper plot), we use the turning point frequency defined in Eq.(82). It depends sensitively on the precision of the measurement. A realistic value is $\sim 10 \%$. For comparison, we also show results for the idealistic case of $1 \%$. These plots include variation of $G \mu$. In the bottom plot of Fig. 23, we show the case of an intermediate inflationary era, which depends very sensitively on the number of efolds, while the turning point frequency does not depend on the duration of the non-standard era for other non-standard cosmologies. The upper plot applies to any non-standard era with equation of state $\rho \sim a^{-n}$ with any $n$ between 3 and 6 , independently of the duration of this non-standard era. Interestingly, radio telescope SKA can be sensitive to a low-scale inflationary era. Note that the bands in Fig. 23 are calculated by neglecting particle production, which will affect mostly ET and CE, c.f. fig. 22 and 19. The lower bound on $T_{\Delta}$ and $E_{\text {inf }}$ should be weaker by 1 order of magnitude for $\mathrm{ET}$ and $\mathrm{CE}$ if there is a cutoff from particle production.

\section{Summary and conclusion}

In standard cosmology, the GW spectrum generated by a network of Nambu-Goto cosmic strings (and mainly due to emission by loops) is nearly scale-invariant. Its potential observation by third-generation interferometers would be a unique probe of new effects beyond the standard models of particle physics and cosmology. Such opportunity was pointed out in [21-25].

Deriving firm conclusions is still premature as theoretical predictions of the GW spectrum from CS are subject to a number of large uncertainties. Still, we feel that the extraordinary potential offered by future GW observatories to probe high energy physics has not yet been explored, and in a series of papers, we are starting to scrutinise how much can be learnt, even if only in the far-future, after those planned GW observatories will have reached their expected long-term sensitivity and the astrophysical foreground will have been fully understood. 

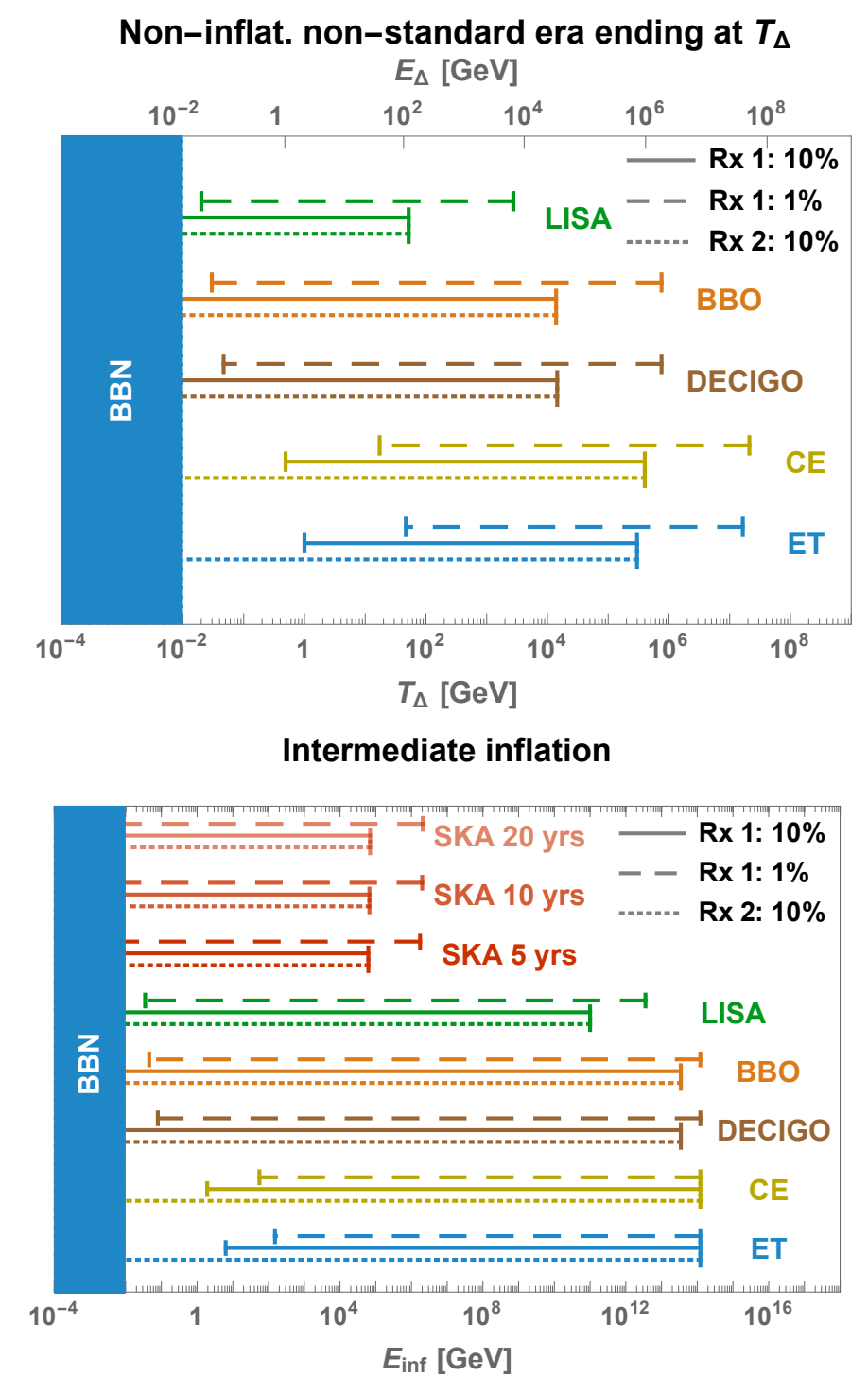

Figure 23: Top: Sensitivity to the energy scale $E_{\Delta}$ of the universe at the end of any noninflationary non-standard era for each future $G W$ interferometer. The connection to $E_{\Delta}$ is given by the observation of the turning-point frequency defined in Eq. (82). The width of the bands includes varying the string tension for $G \mu<10^{-10}$. The dotted, dashed and solid lines correspond to different observational prescriptions defined in Sec. 9.1. Bottom: Sensitivity to the energy scale $E_{\text {inf }}$ of an intermediate inflationary era for each future $G W$ interferometer as well as for future radio telescope SKA. The connection to $E_{\mathrm{inf}}$ is given by the observation of the turning-point frequency defined in Eq. (83). The width of the band also includes varying the number of efolds of inflation $N_{e}$ up to 20.

Deviations in the cosmological history with respect to standard cosmology not only change the redshifting factor of GW but also modify the time of loop formation and the loop-production efficiency. We presented predictions for the resulting GW spectra under a number of assumptions which we have comprehensively reviewed.

We extend previous works in several directions, as listed in the introduction.

A particular feature of gravitational waves from cosmic strings is the relation between the observed frequency and the GW production mechanism. In contrast with shortlasting cosmological sources of gravitational waves, such as phase transitions, where the frequency is simply related to the Hubble radius at the time of GW emission, for cosmic 
strings the time of GW emission is much later than the time of loop production such that the observed frequency is not related to the loop size at the time of loop production but to a much larger length scale. We stressed that a given interferometer may be sensitive to very different energy scales, depending on the nature and duration of the non-standard era, and the value of the string tension. This goes against usual paradigms. For instance, it is customary to talk about LISA as a window on the EW scale [191, 192]. This does not apply for GW from cosmic strings, as LISA could either be a window on a non-standard matter era at the QCD scale or on a $10 \mathrm{TeV}$ inflationary era, meaning that the GW observed in the LISA band have been emitted by loops that were created at the QCD epoch, or at a $10 \mathrm{TeV}$ epoch depending on the nature of the new physics responsible for the non-standard era. Interestingly, the Einstein Telescope and Cosmic Explorer offer a window of observation on the highest scales, up to $10^{14} \mathrm{GeV}$ inflationary eras. They can also be windows on the $\mathrm{EW}$ and $\mathrm{TeV}$ scales, as will be discussed in more details in [53]. BBO/DECIGO could probe new physics in an intermediate range. Finally, radio telescope SKA may be sensitive to a TeV scale inflationary era.

We will apply these findings to probe well-motivated particle physics scenarios in [53]. Particularly generic are intermediate matter eras triggered by cold heavy particles arising in UV completions of the Standard Model. In [53], we show on specific models how a new uncharted particle theory space can be probed from analysis of SGWB from CS.

Finally, one important question will be to work out how to distinguish a stage of matter or inflationary expansion, which both lead to a suppression of the GW spectrum, from the cutoff induced by particle production from small loops. Both predict a cutoff at high frequencies and lead to similar spectra. Interestingly, particle production by cosmic string networks can be probed through cosmic rays and bring complementary nongravitational information on the SGWB. Besides, the complementarity between different GW instruments will be crucial here as the detection of the low-frequency peak of the spectrum (due to the transition from the standard radiation to the standard matter era) can enable to probe the string tension and to break the degeneracy between different spectral predictions. The possibility to reconstruct the spectral shape of a SGWB was analysed in [193] using LISA data only. In the case of a SGWB generated by CS, which can span more than twenty decades in frequency, it will be crucial to use data from different interferometers (and even from radio telescopes) to probe the full spectrum.

\section{Acknowledgements}

We thank Marek Lewicki for useful discussions. We also thank Valerie Domcke and the DESY summer students 2018 Anna Kormu and Sam Wikeley for their participation at an early stage of the project. This work is supported by the Deutsche Forschungsgemeinschaft under Germanys Excellence Strategy - EXC 2121 Quantum Universe - 390833306. The work of Y.G. is partly supported by a PIER Seed Project funding 'Dark Matter at $10 \mathrm{TeV}$ and beyond, a new goal for cosmic-ray experiments' (Project ID PIF-2017-72). P.S. acknowledges his master-degree scholarship from the Development and Promotion of Science and Technology Talents project (DPST), Thailand. 


\section{A Cosmic string detection beyond GW}

By confronting our theoretical predictions for the GW spectrum from CS with the sensitivity curves of EPTA [119] and NANOGrav [120] (which we take from [123]), we derived the respective bounds $G \mu \lesssim 2 \times 10^{-10}$ (EPTA) and $G \mu \lesssim 5 \times 10^{-11}$ (NANOGrav), as discussed in Section 2.4. For this reason, we only considered in our analysis $G \mu$ values smaller than $5 \times 10^{-11}$.

Such bounds are strongly depending on our assumptions for the theoretical prediction of the GW background. In this appendix, we quote other bounds on $G \mu$ which are independent from Pulsar Timing Array limits. They come from gravitational lensing and CMB observables and are much weaker.

\section{A.1 Gravitational lensing}

The presence of energy confined within the core of CS affects the spacetime around them. The metric near a CS is locally flat but globally conical [194]. Photons from a distant celestial object travelling in the vicinity of a CS are subject to gravitational lensing effects. The corresponding constraint $G \mu \lesssim 3 \times 10^{-7}$ has been derived from the search of gravitational lensing signatures of CS in the high-resolution wide-field astronomical surveys GOODS [195] and COSMOS [196]. It has been claimed that constraints from gravitational lensing surveys at radio frequencies like LOFAR and SKA could reach $G \mu \lesssim$ $10^{-9}[197]$.

\section{A.2 Temperature anisotropies in the CMB}

There are two possible effects from CS on temperature fluctuations in the CMB:

1. CS moving through the line-of-sight can induce Doppler shifts on the photons coming from the last scattering surface, known as the Kaiser-Stebbins-Gott effect [198200], potentially leaving line-like discontinuities in the CMB.

2. A CS moving in the primordial plasma leaves surdensity perturbations, the so-called wakes [201], possibly imprinted in the CMB temperature anisotropy. Due to the stochastic behavior of the Kibble mechanism, these perturbations are decoherent and give rise to a CMB spectrum without acoustic peaks [202].

Lattice numerical computation of the temperature anisotropy in Abelian-Higgs [203, 204], Nambu-Goto [202, 205, 206] or global strings [207] have constrained the string tension to $G \mu \lesssim$ few $\times 10^{-7}$ [208]. Constraints of the same magnitude can be found from nongaussianities [208-210]. Also, the same signatures as in the CMB can be imprinted in the $21 \mathrm{~cm}$ power spectrum, and an experiment with a collecting area of $10^{4}-10^{6} \mathrm{~km}^{2}$ might constrain $G \mu \lesssim 10^{-10}-10^{-12}$ [211].

\section{B Derivation of the GW spectrum from CS}

In this appendix we provide the steps leading to equation (26). 


\section{B.1 From GW emission to detection}

The GW energy density spectrum today is defined as

$$
\Omega_{\mathrm{GW}}(f)=\frac{f}{\rho_{c}}\left|\frac{d \rho_{\mathrm{GW}}\left(f, t_{0}\right)}{d f}\right|
$$

After emission, the GW energy density redshifts as radiation, $\rho_{\mathrm{GW}} \propto a^{-4}$, so the GW energy density per unit of frequency redshifts as

$$
\frac{d \rho_{\mathrm{GW}}\left(f, t_{0}\right)}{d f}=\frac{d \rho_{\mathrm{GW}}(\tilde{f}, \tilde{t})}{d \tilde{f}}\left(\frac{a(\tilde{t})}{a\left(t_{0}\right)}\right)^{3}
$$

where the frequency at emission $\tilde{f}$ is related to the frequency today $f$ through

$$
\tilde{f}=\frac{a\left(t_{0}\right)}{a(\tilde{t})} f
$$

\section{B.2 From loop production to GW emission}

After its formation at $t_{i}$, a loop shrinks through emission of GW with a rate $\Gamma G \mu$ so that its length evolves as, c.f. sec. 2.3

$$
l(t)=\alpha t_{i}-\Gamma G \mu\left(t-t_{i}\right)
$$

where $\alpha$ is the length at formation in units of the horizon size. The resulting GW are emitted at a frequency $\tilde{f}$ corresponding to one of the proper modes of the loop, i.e.

$$
\tilde{f}=\frac{2 k}{l}, \quad k \in \mathbb{Z}^{+} .
$$

The GW energy rate emitted by one loop through the mode $k$ is, c.f. sec. 2.3

$$
\frac{d E_{\mathrm{GW}}^{(k)}}{d t}=\Gamma^{(k)} G \mu^{2}, \quad \text { with } \quad \sum_{k} \Gamma^{(k)}=\Gamma,
$$

where

$$
\Gamma^{(k)}=\frac{\Gamma k^{-4 / 3}}{\sum_{p=1}^{\infty} p^{-4 / 3}} \simeq \frac{\Gamma k^{-4 / 3}}{3.60},
$$

which assumes that the GW emission is dominated by cusps. The GW energy density spectrum resulting from the emission of all the decaying loops until today is

$$
\frac{d \rho_{\mathrm{GW}}(\tilde{f}, \tilde{t})}{d \tilde{f}}=\int_{t_{F}}^{t_{0}} d \tilde{t} \frac{d E_{\mathrm{GW}}}{d \tilde{t}} \frac{d n(\tilde{f}, \tilde{t})}{d \tilde{f}},
$$

where $d n(\tilde{f}, \tilde{t}) / d \tilde{f}$ is the number density of loops emitting GW at frequency $\tilde{f}$ at time $\tilde{t}$ and $t_{0}$ is the age of the universe today. Loops start being created at time of CS network formation $t_{F}$, after the damped evolution has stopped, c.f. sec. D. 


\section{B.3 The loop production}

In sec. 3.2 , we assume the loop-formation rate to be

$$
\frac{d n}{d t_{i}}=(0.1) \frac{C_{\mathrm{eff}}\left(t_{i}\right)}{\alpha t_{i}^{4}},
$$

where $C_{\text {eff }}\left(t_{i}\right)$ is the loop-formation efficiency. We deduce the loop number density per unit of frequency

$$
\begin{aligned}
\frac{d n(\tilde{f}, \tilde{t})}{d \tilde{f}} & =\left[\frac{a\left(t_{i}\right)}{a(\tilde{t})}\right]^{3} \frac{d n}{d t_{i}} \cdot \frac{d t_{i}}{d l} \cdot \frac{d l}{d \tilde{f}} \\
& =\left[\frac{a\left(t_{i}\right)}{a(\tilde{t})}\right]^{3} \sum_{k}(0.1) \frac{C_{e f f}\left(t_{i}\right)}{t_{i}^{4}} \cdot \frac{1}{\alpha(\alpha+\Gamma G \mu)} \cdot \frac{2 k}{f^{2}}\left[\frac{a(\tilde{t})}{a\left(t_{o}\right)}\right]^{2}
\end{aligned}
$$

\section{B.4 The master equation}

Finally, we get the GW energy density spectrum

$$
\begin{aligned}
\Omega_{\mathrm{GW}}(f) & =\sum_{k} \Omega_{\mathrm{GW}}^{(k)}(f) \\
& =\sum_{k} \frac{1}{\rho_{c}} \frac{2 k}{f} \frac{(0.1) \Gamma^{(k)} G \mu^{2}}{\alpha(\alpha+\Gamma G \mu)} \int_{t_{F}}^{t_{0}} d \tilde{t} \frac{C_{e f f}\left(t_{i}\right)}{t_{i}^{4}}\left[\frac{a(\tilde{t})}{a\left(t_{0}\right)}\right]^{5}\left[\frac{a\left(t_{i}\right)}{a(\tilde{t})}\right]^{3} \theta\left(t_{i}-t_{F}\right) \theta\left(t_{i}-\frac{l_{*}}{\alpha}\right) .
\end{aligned}
$$

The first Heaviside function stands for the time $t_{\mathrm{F}}$ of formation of the long-string network, and the second one for the time of particle production which is more efficient than GW emission for loops of length smaller than a characteristic length $l_{*}$, which depends on the string small-scale structure, c.f. sec 3.1. The time $t_{i}$ of formation of the loops, which emit at time $\tilde{t}$ and which give the detected frequency $f$, can be determined from eq. (65) and eq. (66)

$$
t_{i}(f, \tilde{t})=\frac{1}{\alpha+\Gamma G \mu}\left[\frac{2 k}{f} \frac{a(\tilde{t})}{a\left(t_{0}\right)}+\Gamma G \mu \tilde{t}\right] .
$$

Note that the contribution coming from the higher modes are related to the contribution of the first mode by

$$
\Omega_{\mathrm{GW}}^{(k)}(f)=k^{-4 / 3} \Omega_{G W}^{(1)}(f / k) .
$$

\section{Derivation of the frequency - temperature relation}

In this appendix, we compute the correspondence between an observed frequency $f$ and the temperature $T$ of the universe when the loops responsible for that frequency have been formed.

\section{C.1 In standard cosmology}

According to the scaling of the loop-formation rate $d n / d t_{i} \propto t_{i}^{-4}$, the main contribution to the GW emission at time $\tilde{t}$ comes from the loops created at the earliest epoch. Correspondingly, loops created at $t_{i}$ contribute to the spectrum as late as possible, at the main 


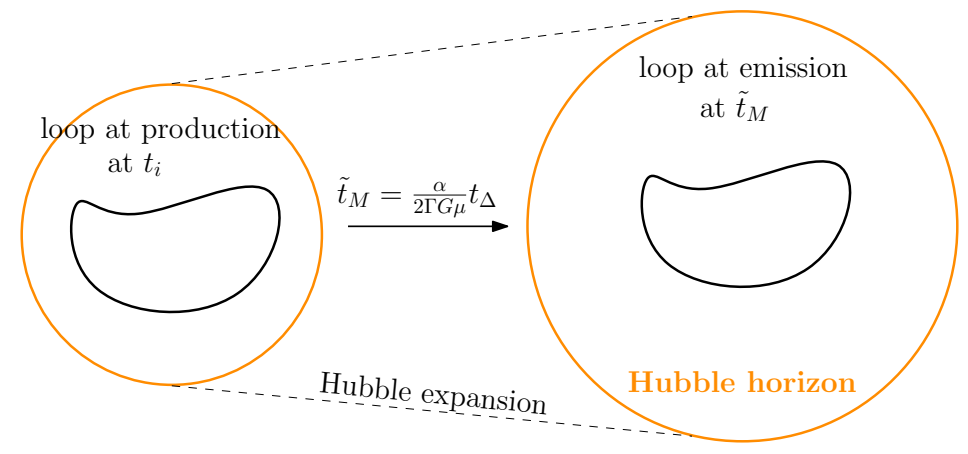

Figure 24: Loops produced at time $t_{i}$ contribute to the $G W$ spectrum much later, when they have accomplished half of their lifetime, at $\tilde{t}_{\mathrm{M}} \simeq \alpha t_{i} /(2 \Gamma G \mu)$. Hence $G W$ emitted from cosmic-string loops are exempt from a redshift factor a $\left(\tilde{t}_{\mathrm{M}}\right) / a\left(t_{i}\right)$ so have much higher frequency than $G W$ produced from other sources at the same energy scale.

emission time $\tilde{t}_{\mathrm{M}}$. The latest emission time is set by the loop lifetime $\alpha t_{i} / \Gamma G \mu$, where $\alpha$ is the loop-length at formation in horizon unit, c.f. eq. (11). Hence, a loop produced at time $t_{i}$ mainly contributes to the spectrum, much later c.f. fig. 24, at a time

$$
\tilde{t}_{\mathrm{M}} \simeq \frac{\alpha t_{i}}{2 \Gamma G \mu}
$$

where the factor $1 / 2$ is found upon maximizing the loop-formation rate $d n / d t_{i} \propto t_{i}^{-4}$ and upon assuming $\alpha \gg \Gamma G \mu$. The loop length after half the loop lifetime, in eq. (76), is equal to half the length at formation $\alpha t_{i} / 2$, c.f. eq. (65). Hence the emitted frequency is set by

$$
\begin{aligned}
\alpha t_{i} & \simeq \frac{4}{f} \frac{a\left(\tilde{t}_{M}\right)}{a\left(t_{0}\right)} \\
& \simeq \frac{4}{f} \frac{a\left(\tilde{t}_{M}\right)}{a\left(t_{\mathrm{eq}}\right)} \frac{a\left(t_{\mathrm{eq}}\right)}{a\left(t_{0}\right)} \\
& \simeq \frac{4}{f}\left(\frac{\tilde{t}_{M}}{t_{\mathrm{eq}}}\right)^{1 / 2}\left(\frac{t_{\mathrm{eq}}}{t_{0}}\right)^{2 / 3}
\end{aligned}
$$

where we used $f a\left(t_{0}\right) / a(\tilde{t})=2 k / l$ and only considered the first Fourier mode $k=1$, c.f. eq. (8). By merging eq. (76) and eq. (79), we obtain the relation between an observed frequency $f$ and the time $t_{i}$ of loop formation

$$
f \simeq \sqrt{\frac{8 z_{\mathrm{eq}}}{\alpha \Gamma G \mu}}\left(\frac{t_{\mathrm{eq}}}{t_{i}}\right)^{1 / 2} t_{0}^{-1},
$$

where the redshift at matter-radiation equality is $z_{\mathrm{eq}}=\Omega_{\mathrm{C}} / \Omega_{\gamma} \simeq 3360$, and $t_{\mathrm{eq}} \simeq$ 51.8 kyrs (from integrating eq. (40)) and $t_{0} \simeq 13.8$ Gyrs [180]. Finally, using entropy conservation, we obtain the relation between the frequency $f$ at observation and the temperature $T$ of the universe when the corresponding loops are formed

$$
\begin{aligned}
f & \simeq \sqrt{\frac{8}{z_{\mathrm{eq}} \alpha \Gamma G \mu}}\left(\frac{g_{*}\left(T_{\Delta}\right)}{g_{*}\left(T_{0}\right)}\right)^{1 / 4}\left(\frac{T}{T_{0}}\right) t_{0}^{-1} \\
& \simeq\left(6.7 \times 10^{-2} \mathrm{~Hz}\right)\left(\frac{T}{\mathrm{GeV}}\right)\left(\frac{0.1 \times 50 \times 10^{-11}}{\alpha \Gamma G \mu}\right)^{1 / 2}\left(\frac{g_{*}\left(T_{i}\right)}{g_{*}\left(T_{0}\right)}\right)^{1 / 4} .
\end{aligned}
$$




\section{C.2 During a change of cosmology}

The derivation of (81) does not take into account the time-variation of $C_{\text {eff }}$. It assumes that loops are produced and decayed during the scaling regime in the radiation era. An observable to test the non-standard cosmology is the frequency $f_{\Delta}$ of the turning-point defined as the frequency at which the GW spectrum starts to deviate from the standardcosmology behavior and the spectral index changes. We obtain different fitted values for this turning point frequency depending on the prescription. We quote below different expressions, depending whether we assume that the spectrum can be measured with a $10 \%$ precision, and $1 \%$ respectively. We compare the predictions obtained using a scaling and VOS network:

$f_{\Delta} \simeq \mathrm{Hz}\left(\frac{T}{\mathrm{GeV}}\right)\left(\frac{0.1 \times 50 \times 10^{-11}}{\alpha \Gamma G \mu}\right)^{1 / 2}\left(\frac{g_{*}(T)}{g_{*}\left(T_{0}\right)}\right)^{1 / 4} \times \begin{cases}2 \times 10^{-3} & \text { for VOS, } 10 \% \\ 45 \times 10^{-3} & \text { for scaling, } 10 \% \\ 0.04 \times 10^{-3} & \text { for VOS, } 1 \% \\ 15 \times 10^{-3} & \text { for scaling, } 1 \%\end{cases}$

Therefore, the turning point frequency is lower in VOS than in scaling by a factor $\sim 22.5$ if we define the turning-point frequency by an amplitude deviation of $10 \%$ with respect to standard cosmology, and by a factor $\sim 375$ for a deviation of $1 \%$. The loops contributing to this part of the spectrum have been formed at the time of the change of cosmology. When the cosmology changes, the network achieves a transient evolution in order to reach the new scaling regime. The long-string network needs extra time to transit from one scaling regime to the other, hence the shift in the relation between observed frequency and temperature of loop formation at the turning-point, c.f. sec. (6.2).

\section{C.3 In the presence of an intermediate inflation period}

The above derivation of the relation between the observed frequency and the time of loop production assumes that cosmic-string loops are constantly being produced throughout the cosmic history. It does not apply if the network experiences an intermediate era of inflation. This case is discussed in sec. 8.2 and the turning-point formulae are, for a given precision

$$
f_{\Delta} \simeq \mathrm{Hz}\left(\frac{T}{\mathrm{GeV}}\right)\left(\frac{0.1 \times 50 \times 10^{-11}}{\alpha \Gamma G \mu}\right)^{1 / 2}\left(\frac{g_{*}(T)}{g_{*}\left(T_{0}\right)}\right)^{1 / 4} \times \begin{cases}1.5 \times 10^{-4} & \text { for } 10 \% \\ 5 \times 10^{-6} & \text { for } 1 \%\end{cases}
$$

\section{C.4 Cut-off from particle production}

The cutoff frequency due to particle production is given in sec. 3.4.

\section{Derivation of the VOS equations}

\section{D.1 The NG string in an expanding universe}

The Velocity-dependent One-Scale equations (VOS) in eq. (34), describe the evolution of a network of long strings in term of the mean velocity $\bar{v}$ and the correlation length 
$\xi=L / t$, see the original papers $[175,177,178]$ or the recent review [179]. The set of points visited by the $\mathrm{NG}$ string during its time evolution form a $2 \mathrm{D}$ manifold, called the world-sheet, described by time-like and space-like coordinates $t$ and $\sigma$. The embedding of the $2 \mathrm{D}$ world-sheet in the $4 \mathrm{D}$ space-time is described by $x^{\mu}(\mathrm{t}, \sigma)$ where $\mu=1,2,3,4$. The choice of the word-sheet coordinates being arbitrary, we have two gauge degrees of freedom which we can fix by imposing $\dot{\mathbf{x}} \cdot \mathbf{x}^{\prime}=0$ and $\mathrm{t}=\tau$ where $\tau$ is the conformal time of the expanding universe. The dot and prime denote the derivatives with respect to the time-like and space-like world-sheet coordinates, $\dot{\mathbf{x}} \equiv d \mathbf{x} / d \mathbf{t}$ and $\mathbf{x}^{\prime} \equiv d \mathbf{x} / d \sigma$. Then, the equations of motion of the NG string in a FRW universe are [220]

$$
\begin{aligned}
\ddot{\mathbf{x}}+2 \mathcal{H}\left(1-\dot{\mathrm{x}}^{2}\right) \dot{\mathrm{x}} & =\frac{1}{\epsilon}\left(\frac{\mathrm{x}^{\prime}}{\epsilon}\right)^{\prime}, \\
\dot{\epsilon}+2 \mathcal{H} \dot{\mathrm{x}}^{2} \epsilon & =0
\end{aligned}
$$

where $\mathcal{H} \equiv \dot{a} / a=H a$ and $\epsilon \equiv \sqrt{\mathbf{x}^{2} /\left(1-\dot{\mathbf{x}}^{2}\right)}$ is the coordinate energy per unit of length.

\section{D.2 The long-string network}

The macroscopic evolution of the long string network can be described by the energy density

$$
\rho_{\infty}=\frac{E}{a^{3}}=\frac{\mu}{a^{2}(\tau)} \int \epsilon d \sigma \equiv \frac{\mu}{L^{2}},
$$

and the root-mean-square averaged velocity

$$
\bar{v}^{2} \equiv\left\langle\dot{\mathbf{x}}^{2}\right\rangle=\frac{\int \dot{\mathbf{x}}^{2} \epsilon d \sigma}{\int \epsilon d \sigma},
$$

where we recall that $\mu$ is the CS linear mass density.

\section{D.3 VOS 1: the correlation length}

Differentiating eq. (86) gives the evolution of the energy density in an expanding universe

$$
\begin{aligned}
\frac{d \rho_{\infty}}{d t} & =\frac{d \rho_{\infty}}{d \tau} \cdot \frac{d \tau}{d t}=\frac{1}{a} \cdot \frac{d \rho_{\infty}}{d \tau}, \\
& =\frac{\mu}{a}\left[\frac{d}{d \tau}\left(\frac{1}{a^{2}}\right) \int \epsilon d \sigma+\frac{1}{a^{2}} \int \frac{d \epsilon}{d \tau} d \sigma\right], \\
& =-2 \frac{\mu}{a^{3}} \mathcal{H}\left[\int \epsilon d \sigma+\int \dot{\mathbf{x}}^{2} \epsilon d \sigma\right], \\
& =-2 H \rho_{\infty}\left(1+\bar{v}^{2}\right) .
\end{aligned}
$$

Moreover, after each string crossing, the network transfers energy into loops with a rate given by eq. (32) and we get

$$
\frac{d \rho_{\infty}}{d t}=-2 H \rho_{\infty}\left(1+\bar{v}^{2}\right)-\tilde{c} \bar{v} \frac{\rho_{\infty}}{L},
$$

which after using eq. (86), leads to the first VOS equation

$$
\operatorname{VOS} 1: \quad \frac{d L}{d t}=H L\left(1+\bar{v}^{2}\right)+\frac{1}{2} \tilde{c} \bar{v} .
$$


We neglect the back-reaction on long strings from gravitational emission which is suppressed with respect to the loop-chopping loss term by $O(G \mu)$. In our study, we do not consider the case of global strings, for which however, the back-reaction due to particle production may play a role.

\section{D.4 Thermal friction}

In addition to the Hubble friction, there can be friction due to the interactions of the strings with particles of the plasma, leading to the retarding force [181]

$$
F=\rho \sigma \bar{v}=\beta T^{3} \bar{v}
$$

where $\rho \sim T^{4}$ is the plasma energy density and $\sigma \sim T^{-1}$ is the cross-section per unit of length. The friction term becomes

$$
2 H \bar{v}^{2} \longrightarrow \frac{\bar{v}^{2}}{l_{d}} \equiv 2 H \bar{v}^{2}+\frac{\bar{v}^{2}}{l_{f}}
$$

where we introduced an effective friction length $l_{d}$ and where the friction length due to particle scattering reads $l_{f} \equiv \mu /(\sigma \rho)=\mu /\left(\beta T^{3}\right)$. At large temperature, the large damping due to the frictional force prevents the CS network to reach the scaling regime until it becomes sub-dominant when $H \gtrsim 1 / l_{f}$, so after the time

$$
t_{*} \sim(G \mu)^{-2} t_{\mathrm{pl}} \sim(G \mu)^{-1} t_{F},
$$

which corresponds to the temperature $T_{*} \simeq 10 \mathrm{GeV}$ for $G \mu=10^{-17}, T_{*} \simeq 1 \mathrm{TeV}$ for $G \mu=10^{-15}, T_{*} \simeq 10 \mathrm{TeV}$ for $G \mu=10^{-13}$, hence respectively impacting the SGWB only above the frequencies $2 \mathrm{kHz}, 200 \mathrm{kHz}, 20 \mathrm{MHz}$, c.f. eq. (27), which are outside the GW interferometer windows, c.f. fig. 4 . Hence we can safely set $l_{f} \rightarrow 0$.

\section{D.5 VOS 2: the mean velocity}

Differentiating eq. (87) gives the evolution of the averaged velocity, which constitutes the second VOS equation

$$
\operatorname{VOS} 2: \quad \frac{d \bar{v}}{d t}=\left(1-\bar{v}^{2}\right)\left[\frac{k(\bar{v})}{L}-\frac{\bar{v}}{l_{d}}\right]
$$

with

$$
k(\bar{v}) \equiv \frac{\left\langle\left(1-\dot{\mathbf{x}}^{2}\right)(\dot{\mathbf{x}} \cdot \mathbf{u})\right\rangle}{\bar{v}\left(1-\bar{v}^{2}\right)},
$$

where $\mathbf{u}$ is the unit vector aligned with the radius of curvature $\propto d^{2} \mathbf{x} / d \sigma^{2} . k(\bar{v})$ indicates the degree of wiggliness of the string. More precisely, $k(\bar{v})=1$ for a straight string and $k(\bar{v}) \lesssim 1$ once we add small-scale structures. We use the results from numerical simulations [175]

$$
k(\bar{v})=\frac{2 \sqrt{2}}{\pi}\left(1-\bar{v}^{2}\right)\left(1+2 \sqrt{2} \bar{v}^{3}\right) \frac{1-8 \bar{v}^{6}}{1+8 \bar{v}^{6}} .
$$

Eq. (97) is a relativistic generalization of Newton's law where the string is accelerated by its curvature $1 / L$ but is damped by the Hubble expansion and plasma friction after a typical length $1 / l_{d}$. 

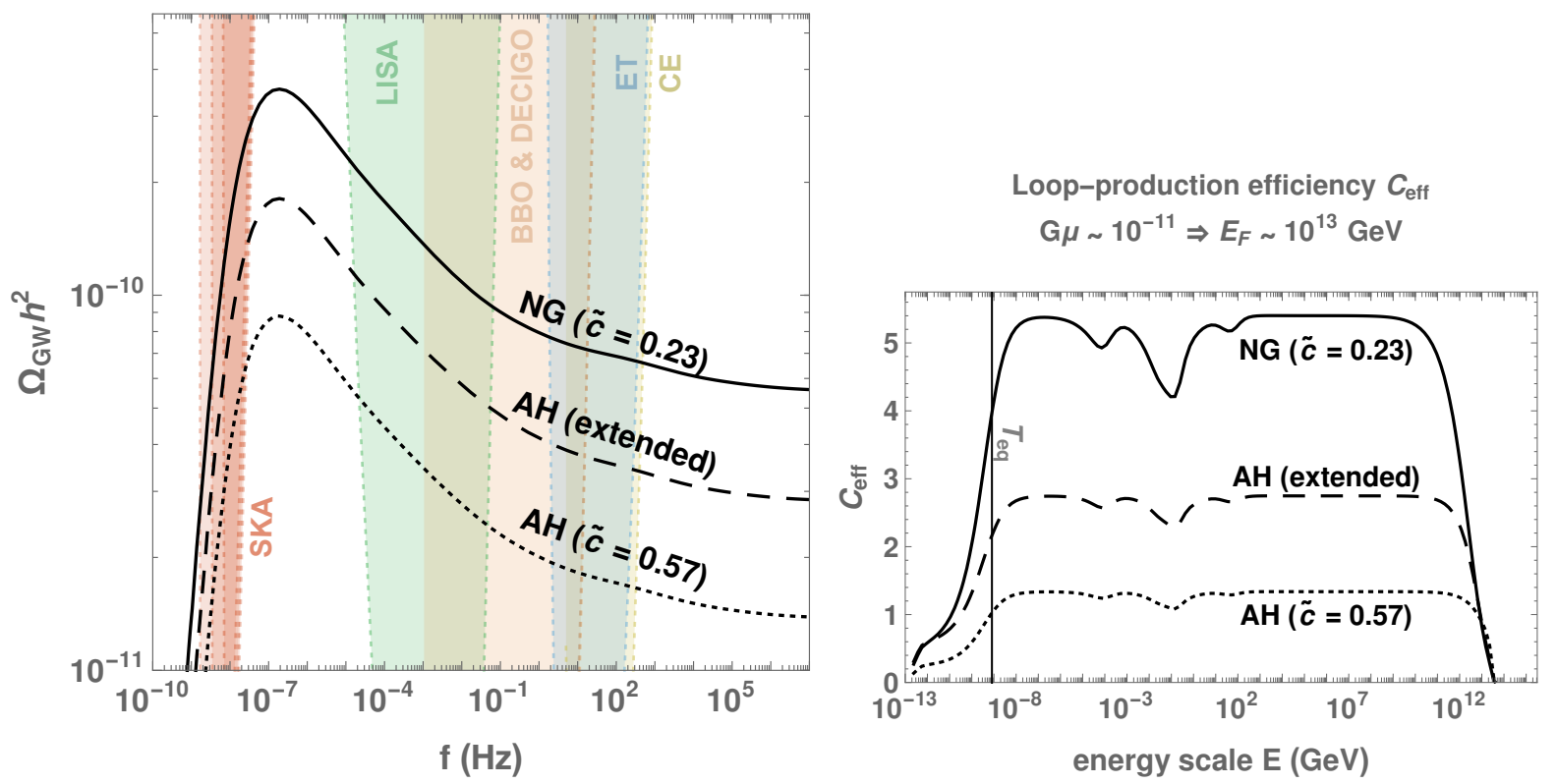

Figure 25: Left: GW spectra with different VOS modellings of the long-string network evolution. The VOS models are either based on NG simulations (solid line $-\tilde{c}=0.23$ ) [175] or abelian-Higgs (AH) field theory simulations (dashed line - $\tilde{c}=0.57)$ [132, 221], possibly extended to include particle production [176] (dotted line). Right: The corresponding loop-production efficiency for each VOS model.

\section{E Extension of the original VOS model}

\section{E.1 VOS model from NG simulations}

In our study, we describe the evolution of the long-string network through the VOS model, defined by the equations in eq. (34). The only free parameter of the model is the loop-chopping efficiency $\tilde{c}$, which is computed to be

$$
\text { NG: } \quad \tilde{c}=0.23 \pm 0.04
$$

from NG network simulations in an expanding universe [175].

\section{E.2 VOS model from AH simulations}

Abelian-Higgs (AH) field theory simulations in both expanding and flat spacetime suggest a larger value $[132,221]$

$$
\text { AH: } \quad \tilde{c}=0.57 \pm 0.04 .
$$

Indeed, in AH simulation, no loops are produced below the string core size so the energy loss into loop formation is lower. Consequently, the loop-chopping efficiency must be increased to maintain scaling.

\section{E.3 VOS model from AH simulations with particle production}

In $\mathrm{AH}$ simulations, the loops produced at the string core scale are non-linear lumps of field, called "proto-loops", which decay fast into massive radiation. Therefore, a recent 


\begin{tabular}{|c|c|c|c|}
\hline Scaling in RD & $\begin{array}{c}\mathrm{NG} \\
\tilde{c}=0.23\end{array}$ & $\begin{array}{c}\mathrm{AH} \\
\tilde{c}=0.57\end{array}$ & $\begin{array}{c}\text { AH extended } \\
\tilde{c}=0.31 \\
\left(d, k_{0}, r, q, \beta\right)\end{array}$ \\
\hline $\bar{v}$ & 0.66 & 0.62 & 0.59 \\
\hline$\xi$ & 0.27 & 0.57 & 0.36 \\
\hline$C_{\text {eff }}$ & 5.4 & 1.3 & 2.8 \\
\hline
\end{tabular}

Table 1: Values of mean velocity $\bar{v}$, correlation length $\xi$, and loop-production efficiency $C_{\text {eff }}$ in radiation scaling regime with different VOS calibrations.

work [176] extends the VOS model by including a term in eq. (93) to account for the emission of massive radiation at the string core scale. The energy-loss function $F(v)$ is modified as

$$
\left.F(\bar{v})\right|_{\text {original }}=\left.\frac{\tilde{c} \bar{v}}{2} \Rightarrow F(\bar{v})\right|_{\text {extended }}=\frac{\tilde{c} \bar{v}+d\left[k_{0}-k(\bar{v})\right]^{r}}{2},
$$

and the momentum operator $k(v)$, c.f. eq. (99), accounting for the amount of small-scale structures in the string, is modified to

$$
k(\bar{v})=\left.\frac{2 \sqrt{2}}{\pi} \frac{1-8 \bar{v}^{6}}{1+8 \bar{v}^{6}} \Rightarrow k(\bar{v})\right|_{\text {extended }}=k_{0} \frac{1-\left(q \bar{v}^{2}\right)^{\beta}}{1+\left(q \bar{v}^{2}\right)^{\beta}},
$$

where more free parameters have been introduced. With $\mathrm{AH}$ simulations, one finds

$$
\text { AH extended: } \quad \tilde{c}=0.31
$$

as well as $d=0.26, k_{0}=1.27, r=1.66, q=2.27$, and $\beta=1.54$. In AH extended, the loop-chopping efficiency, c.f. eq. (104), is smaller than the one in the original AH model, c.f. eq. (101). Indeed, because of the additional energy loss through massive-radiation, less energy loss via loop-chopping is needed to maintain scaling.

In fig. 25, we compare the GW spectra in the different VOS models. The difference in amplitude comes from the difference in the number of loops, set by $C_{\text {eff }}$. The larger the loop-chopping efficiency $\tilde{c}$, the smaller the loop-formation efficiency $C_{\text {eff }}$. This counterintuitive result can be better understood by looking at table. 1. A larger loop-chopping efficiency $\tilde{c}$ implies a larger loop formation rate only during the transient regime. In the scaling regime, a larger loop-chopping efficiency $\tilde{c}$ implies a more depleted long-string network and then a larger correlation length $\xi$. Hence, the long-string network is more sparse and so the rate of loop formation via string crossing is lower.

\section{F Sensitivity curves of GW detectors}

\section{F.1 The signal-to-noise ratio}

The total output of a detector is given by the GW signal plus the noise, $h(t)+n(t)$ where the level of noise $n(t)$ is measured by its noise spectral density $S_{n}(f)$ [1].

$$
\left\langle\tilde{n}^{*}(f) \tilde{n}\left(f^{\prime}\right)\right\rangle \equiv \delta\left(f-f^{\prime}\right) S_{n}(f) .
$$


We define the detector sensitivity $\Omega_{\text {sens }}(f)$ as the magnitude of the SGWB energy density which would mimick the noise spectral density $S_{n}(f)$

$$
\Omega_{\mathrm{sens}}(f)=\frac{2 \pi^{2}}{3 H_{0}^{2}} f^{3} S_{n}(f) .
$$

The capability of an interferometer to detect a SGWB of energy density $\Omega_{\mathrm{GW}}(f)$ after an observation time $T$ is measured by the signal-to-noise ratio (SNR) [222]

$$
\mathrm{SNR}=\sqrt{T \int_{f_{\min }}^{f_{\max }} d f\left[\frac{\Omega_{\mathrm{GW}}(f)}{\Omega_{\mathrm{sens}}(f)}\right]^{2}}
$$

\section{F.2 The power-law integrated sensitivity curve}

Assuming a power law spectrum

$$
\Omega_{\mathrm{GW}}(f)=\Omega_{\beta}\left(\frac{f}{f_{\mathrm{ref}}}\right)^{\beta},
$$

with spectral index $\beta$, amplitude $\Omega_{\beta}$ and reference frequency $f_{\text {ref }}$, we deduce from eq. (107) the amplitude $\Omega_{\beta}$ needed to reach a given SNR after a given observation time $T$

$$
\Omega_{\beta}=\frac{\mathrm{SNR}}{\sqrt{T}}\left(\int_{f_{\min }}^{f_{\max }} d f\left[\frac{h^{2}}{h^{2} \Omega_{\mathrm{sens}}(f)}\left(\frac{f}{f_{\mathrm{ref}}}\right)^{\beta}\right]^{2}\right)^{-1 / 2},
$$

which upon re-injecting into eq. (108) gives

$$
h^{2} \Omega_{\mathrm{GW}}(f)=f^{\beta} \frac{\mathrm{SNR}}{\sqrt{T}}\left(\int_{f_{\min }}^{f_{\max }} d f\left[\frac{f^{\beta}}{h^{2} \Omega_{\mathrm{sens}}(f)}\right]^{2}\right)^{-1 / 2} .
$$

For a given pair (SNR, $T$ ), one obtains a series in $\beta$ of power-law integrated curves. One defines the power-law integrated sensitivity curve $\Omega_{P I}(f)$ as the envelope of those functions [223]

$$
\Omega_{P I}(f) \equiv \max _{\beta}\left[f^{\beta} \frac{\mathrm{SNR}}{\sqrt{T}}\left(\int_{f_{\min }}^{f_{\max }} d f\left[\frac{f^{\beta}}{h^{2} \Omega_{\mathrm{sens}}(f)}\right]^{2}\right)^{-1 / 2}\right] .
$$

Any SGWB signal $\Omega_{\mathrm{GW}}(f)$ which lies above $\Omega_{P I}(f)$ would gives a signal to noise ratio $>$ SNR after an observation time $T$.

\section{F.3 Results}

For the purpose of our study, we computed the power-law integrated sensitivity curve $\Omega_{P I}(f)$, starting from the noise spectral density in [98] for ET, [100] for CE and [96] for BBO/DECIGO. For pulsar timing arrays EPTA, NANOGrav and SKA, we directly took the sensitivity curves from [123]. The signal-to-noise ratio can be improved by using crosscorrelation between multiple detectors, e.g. LIGO-Hanford, LIGO-Livingston, VIRGO but also KAGRA which may join the network at the end of run O3, which began on 
the $1^{\text {st }}$ of April 2019, or LIGO-India which may be operational for run O5 [224]. We computed the SNR for LIGO from the expression [223]

$$
\mathrm{SNR}=\left[2 T \int_{f_{\min }}^{f_{\max }} d f \frac{\Gamma^{2}(f) S_{\mathrm{h}}^{2}(f)}{S_{n}^{1}(f) S_{n}^{2}(f)}\right]^{1 / 2},
$$

where $S_{n}^{1}$ and $S_{n}^{2}$ are the noise spectral densities of the detectors in Hanford and in Livingston for the runs $02, \mathrm{O} 4$ or $\mathrm{O} 5$ and $\Gamma(f)$ is the overlap function between the two LIGO detectors which we took from [225]. The GW power spectral density $S_{h}(f)$ is related to the GW energy density through

$$
S_{h}(f)=\frac{3 H_{0}^{2}}{2 \pi^{2}} \frac{\Omega_{\mathrm{GW}}(f)}{f^{3}} .
$$

We fixed the signal-to-noise ratio $\mathrm{SNR}=10$ and the observational time $T=268$ days for LIGO O2, 1 year for LIGO O4 and O5, and 10 years for other sensitivity curves.

As this paper was completed, Ref. [226] appeared, where the sensitivity curves may differ from us by a factor of order 1 .

\section{References}

[1] C. Caprini and D. G. Figueroa, Cosmological Backgrounds of Gravitational Waves, Class. Quant. Grav. 35 (2018) 163001, [1801.04268].

[2] M. Giovannini, Gravitational waves constraints on postinflationary phases stiffer than radiation, Phys. Rev. D58 (1998) 083504, [hep-ph/9806329].

[3] M. Giovannini, Stochastic backgrounds of relic gravitons: a theoretical appraisal, PMC Phys. A4 (2010) 1, [0901.3026].

[4] A. Riazuelo and J.-P. Uzan, Quintessence and gravitational waves, Phys. Rev. D62 (2000) 083506, [astro-ph/0004156].

[5] V. Sahni, M. Sami and T. Souradeep, Relic gravity waves from brane world inflation, Phys. Rev. D65 (2002) 023518, [gr-qc/0105121].

[6] N. Seto and J. Yokoyama, Probing the equation of state of the early universe with a space laser interferometer, J. Phys. Soc. Jap. 72 (2003) 3082-3086, [gr-qc/0305096].

[7] H. Tashiro, T. Chiba and M. Sasaki, Reheating after quintessential inflation and gravitational waves, Class. Quant. Grav. 21 (2004) 1761-1772, [gr-qc/0307068].

[8] K. Nakayama, S. Saito, Y. Suwa and J. Yokoyama, Space laser interferometers can determine the thermal history of the early Universe, Phys. Rev. D77 (2008) 124001, [0802.2452].

[9] K. Nakayama, S. Saito, Y. Suwa and J. Yokoyama, Probing reheating temperature of the universe with gravitational wave background, JCAP 0806 (2008) 020, [0804.1827].

[10] R. Durrer and J. Hasenkamp, Testing Superstring Theories with Gravitational Waves, Phys. Rev. D84 (2011) 064027, [1105.5283]. 
[11] S. Kuroyanagi, K. Nakayama and S. Saito, Prospects for determination of thermal history after inflation with future gravitational wave detectors, Phys. Rev. D84 (2011) 123513, [1110.4169].

[12] S. Kuroyanagi, T. Chiba and T. Takahashi, Probing the Universe through the Stochastic Gravitational Wave Background, JCAP 1811 (2018) 038, [1807.00786].

[13] R. Jinno, T. Moroi and K. Nakayama, Probing dark radiation with inflationary gravitational waves, Phys. Rev. D86 (2012) 123502, [1208.0184].

[14] P. D. Lasky et al., Gravitational-wave cosmology across 29 decades in frequency, Phys. Rev. X6 (2016) 011035, [1511.05994].

[15] B. Li, P. R. Shapiro and T. Rindler-Daller, Bose-Einstein-condensed scalar field dark matter and the gravitational wave background from inflation: new cosmological constraints and its detectability by LIGO, Phys. Rev. D96 (2017) 063505, [1611.07961].

[16] K. Saikawa and S. Shirai, Primordial gravitational waves, precisely: The role of thermodynamics in the Standard Model, JCAP 1805 (2018) 035, [1803.01038].

[17] R. R. Caldwell, T. L. Smith and D. G. E. Walker, Using a Primordial Gravitational Wave Background to Illuminate New Physics, 1812.07577.

[18] N. Bernal and F. Hajkarim, Primordial Gravitational Waves in Non-standard Cosmologies, 1905.10410.

[19] D. G. Figueroa and E. H. Tanin, Ability of LIGO and LISA to probe the equation of state of the early Universe, 1905.11960.

[20] F. D'Eramo and K. Schmitz, Imprint of a scalar era on the primordial spectrum of gravitational waves, 1904.07870 .

[21] Y. Cui, M. Lewicki, D. E. Morrissey and J. D. Wells, Cosmic Archaeology with Gravitational Waves from Cosmic Strings, Phys. Rev. D97 (2018) 123505, [1711.03104].

[22] Y. Cui, M. Lewicki, D. E. Morrissey and J. D. Wells, Probing the pre-BBN universe with gravitational waves from cosmic strings, 1808.08968.

[23] P. Auclair et al., Probing the gravitational wave background from cosmic strings with LISA, 1909.00819.

[24] G. S. F. Guedes, P. P. Avelino and L. Sousa, Signature of inflation in the stochastic gravitational wave background generated by cosmic string networks, Phys. Rev. D98 (2018) 123505, [1809.10802].

[25] C.-F. Chang and Y. Cui, Stochastic Gravitational Wave Background from Global Cosmic Strings, 1910.04781.

[26] A. Vilenkin, Gravitational radiation from cosmic strings, Phys. Lett. 107B (1981) 47-50.

[27] C. J. Hogan and M. J. Rees, Gravitational interactions of cosmic strings, Nature 311 (1984) 109-113.

[28] T. Vachaspati and A. Vilenkin, Gravitational Radiation from Cosmic Strings, Phys. Rev. D31 (1985) 3052. 
[29] F. S. Accetta and L. M. Krauss, The stochastic gravitational wave spectrum resulting from cosmic string evolution, Nucl. Phys. B319 (1989) 747-764.

[30] D. P. Bennett and F. R. Bouchet, Constraints on the gravity wave background generated by cosmic strings, Phys. Rev. D43 (1991) 2733-2735.

[31] R. R. Caldwell and B. Allen, Cosmological constraints on cosmic string gravitational radiation, Phys. Rev. D45 (1992) 3447-3468.

[32] B. Allen and E. P. S. Shellard, Gravitational radiation from cosmic strings, Phys. Rev. D45 (1992) 1898-1912.

[33] R. A. Battye, R. R. Caldwell and E. P. S. Shellard, Gravitational waves from cosmic strings, in Topological defects in cosmology, pp. 11-31, 1997. astro-ph/9706013.

[34] M. R. DePies and C. J. Hogan, Stochastic Gravitational Wave Background from Light Cosmic Strings, Phys. Rev. D75 (2007) 125006, [astro-ph/0702335].

[35] X. Siemens, V. Mandic and J. Creighton, Gravitational wave stochastic background from cosmic (super)strings, Phys. Rev. Lett. 98 (2007) 111101, [astro-ph/0610920].

[36] S. Olmez, V. Mandic and X. Siemens, Gravitational-Wave Stochastic Background from Kinks and Cusps on Cosmic Strings, Phys. Rev. D81 (2010) 104028, [1004.0890].

[37] T. Regimbau, S. Giampanis, X. Siemens and V. Mandic, The stochastic background from cosmic (super)strings: popcorn and (Gaussian) continuous regimes, Phys. Rev. D85 (2012) 066001, [1111.6638].

[38] S. A. Sanidas, R. A. Battye and B. W. Stappers, Constraints on cosmic string tension imposed by the limit on the stochastic gravitational wave background from the European Pulsar Timing Array, Phys. Rev. D85 (2012) 122003, [1201.2419].

[39] S. A. Sanidas, R. A. Battye and B. W. Stappers, Projected constraints on the cosmic (super)string tension with future gravitational wave detection experiments, Astrophys. J. 764 (2013) 108, [1211.5042].

[40] P. Binetruy, A. Bohe, C. Caprini and J.-F. Dufaux, Cosmological Backgrounds of Gravitational Waves and eLISA/NGO: Phase Transitions, Cosmic Strings and Other Sources, JCAP 1206 (2012) 027, [1201.0983].

[41] S. Kuroyanagi, K. Miyamoto, T. Sekiguchi, K. Takahashi and J. Silk, Forecast constraints on cosmic string parameters from gravitational wave direct detection experiments, Phys. Rev. D86 (2012) 023503, [1202.3032].

[42] S. Kuroyanagi, K. Miyamoto, T. Sekiguchi, K. Takahashi and J. Silk, Forecast constraints on cosmic strings from future $C M B$, pulsar timing and gravitational wave direct detection experiments, Phys. Rev. D87 (2013) 023522, [1210.2829].

[43] P. Creminelli, A. Nicolis and R. Rattazzi, Holography and the electroweak phase transition, JHEP 03 (2002) 051, [hep-th/0107141].

[44] L. Randall and G. Servant, Gravitational waves from warped spacetime, JHEP 05 (2007) 054, [hep-ph/0607158].

[45] G. Nardini, M. Quiros and A. Wulzer, A Confining Strong First-Order Electroweak Phase Transition, JHEP 09 (2007) 077, [0706.3388]. 
[46] T. Konstandin and G. Servant, Cosmological Consequences of Nearly Conformal Dynamics at the TeV scale, JCAP 1112 (2011) 009, [1104.4791].

[47] B. von Harling and G. Servant, QCD-induced Electroweak Phase Transition, JHEP 01 (2018) 159, [1711.11554].

[48] S. Iso, P. D. Serpico and K. Shimada, QCD-Electroweak First-Order Phase Transition in a Supercooled Universe, Phys. Rev. Lett. 119 (2017) 141301, [1704.04955].

[49] S. Bruggisser, B. Von Harling, O. Matsedonskyi and G. Servant, Electroweak Phase Transition and Baryogenesis in Composite Higgs Models, JHEP 12 (2018) 099, [1804.07314].

[50] P. Baratella, A. Pomarol and F. Rompineve, The Supercooled Universe, JHEP 03 (2019) $100,[1812.06996]$.

[51] D. Matsunami, L. Pogosian, A. Saurabh and T. Vachaspati, Decay of Cosmic String Loops Due to Particle Radiation, Phys. Rev. Lett. 122 (2019) 201301, [1903.05102].

[52] P. Auclair, D. A. Steer and T. Vachaspati, Particle emission and gravitational radiation from cosmic strings: observational constraints, 1911.12066.

[53] Y. Gouttenoire, G. Servant and P. Simakachorn, BSM with Cosmic Strings: Heavy, up to EeV mass, Unstable Particles, 1912.XXXXX.

[54] T. W. B. Kibble, Topology of Cosmic Domains and Strings, J. Phys. A9 (1976) 1387-1398.

[55] M. B. Hindmarsh and T. W. B. Kibble, Cosmic strings, Rept. Prog. Phys. 58 (1995) 477-562, [hep-ph/9411342].

[56] A. Vilenkin and E. P. S. Shellard, Cosmic Strings and Other Topological Defects. Cambridge University Press, 2000.

[57] T. Vachaspati, L. Pogosian and D. Steer, Cosmic Strings, Scholarpedia 10 (2015) 31682, [1506.04039].

[58] E. Witten, Cosmic Superstrings, Phys. Lett. 153B (1985) 243-246.

[59] G. Dvali and A. Vilenkin, Formation and evolution of cosmic D strings, JCAP 0403 (2004) 010, [hep-th/0312007].

[60] E. J. Copeland, R. C. Myers and J. Polchinski, Cosmic F and D strings, JHEP 06 (2004) 013, [hep-th/0312067].

[61] J. Polchinski, Introduction to cosmic F-and D-strings, in String theory: From gauge interactions to cosmology. Proceedings, NATO Advanced Study Institute, Cargese, France, June 7-19, 2004, pp. 229-253, 2004. hep-th/0412244.

[62] M. Sakellariadou, Cosmic Superstrings, Phil. Trans. Roy. Soc. Lond. A366 (2008) 2881-2894, [0802.3379].

[63] A.-C. Davis, P. Brax and C. van de Bruck, Brane Inflation and Defect Formation, Phil. Trans. Roy. Soc. Lond. A366 (2008) 2833-2842, [0803.0424].

[64] M. Sakellariadou, Cosmic Strings and Cosmic Superstrings, Nucl. Phys. Proc. Suppl. 192-193 (2009) 68-90, [0902.0569]. 
[65] E. J. Copeland and T. W. B. Kibble, Cosmic Strings and Superstrings, Proc. Roy. Soc. Lond. A466 (2010) 623-657, [0911.1345].

[66] R. Jeannerot, J. Rocher and M. Sakellariadou, How generic is cosmic string formation in SUSY GUTs, Phys. Rev. D68 (2003) 103514, [hep-ph/0308134].

[67] M. Sakellariadou, Production of Topological Defects at the End of Inflation, Lect. Notes Phys. 738 (2008) 359-392, [hep-th/0702003].

[68] W. Buchmüller, V. Domcke, K. Kamada and K. Schmitz, The Gravitational Wave Spectrum from Cosmological B - L Breaking, JCAP 1310 (2013) 003, [1305.3392].

[69] J. A. Dror, T. Hiramatsu, K. Kohri, H. Murayama and G. White, Testing Seesaw and Leptogenesis with Gravitational Waves, 1908.03227.

[70] H. B. Nielsen and P. Olesen, Vortex Line Models for Dual Strings, Nucl. Phys. B61 (1973) 45-61.

[71] R. J. Scherrer and J. A. Frieman, COSMIC STRINGS AS RANDOM WALKS, Phys. Rev. D33 (1986) 3556.

[72] E. P. S. Shellard, Cosmic String Interactions, Nucl. Phys. B283 (1987) 624-656.

[73] M. Eto, K. Hashimoto, G. Marmorini, M. Nitta, K. Ohashi and W. Vinci, Universal Reconnection of Non-Abelian Cosmic Strings, Phys. Rev. Lett. 98 (2007) 091602, [hep-th/0609214].

[74] M. G. Jackson, N. T. Jones and J. Polchinski, Collisions of cosmic F and D-strings, JHEP 10 (2005) 013, [hep-th/0405229].

[75] E. J. Copeland, T. W. B. Kibble and D. A. Steer, Constraints on string networks with junctions, Phys. Rev. D75 (2007) 065024, [hep-th/0611243].

[76] P. Laguna and R. A. Matzner, PEELING U(1) GAUGE COSMIC STRINGS, Phys. Rev. Lett. 62 (1989) 1948-1951.

[77] A. Achucarro and R. de Putter, Effective non-intercommutation of local cosmic strings at high collision speeds, Phys. Rev. D74 (2006) 121701, [hep-th/0605084].

[78] T. W. B. Kibble, Evolution of a system of cosmic strings, Nucl. Phys. B252 (1985) 227.

[79] D. P. Bennett and F. R. Bouchet, Evidence for a Scaling Solution in Cosmic String Evolution, Phys. Rev. Lett. 60 (1988) 257.

[80] D. P. Bennett and F. R. Bouchet, Cosmic string evolution, Phys. Rev. Lett. 63 (1989) 2776.

[81] A. Albrecht and N. Turok, Evolution of Cosmic String Networks, Phys. Rev. D40 (1989) 973-1001.

[82] B. Allen and E. P. S. Shellard, Cosmic string evolution: a numerical simulation, Phys. Rev. Lett. 64 (1990) 119-122.

[83] V. B. Klaer and G. D. Moore, The dark-matter axion mass, JCAP 1711 (2017) 049, [1708.07521]. 
[84] M. Gorghetto, E. Hardy and G. Villadoro, Axions from Strings: the Attractive Solution, JHEP 07 (2018) 151, [1806.04677].

[85] M. Kawasaki, T. Sekiguchi, M. Yamaguchi and J. Yokoyama, Long-term dynamics of cosmological axion strings, PTEP 2018 (2018) 091E01, [1806.05566].

[86] A. Vaquero, J. Redondo and J. Stadler, Early seeds of axion miniclusters, 1809.09241.

[87] M. Buschmann, J. W. Foster and B. R. Safdi, Early-Universe Simulations of the Cosmological Axion, 1906.00967.

[88] C. J. A. P. Martins, Scaling properties of cosmological axion strings, Phys. Lett. B788 (2019) 147-151, [1811.12678].

[89] M. Hindmarsh, J. Lizarraga, A. Lopez-Eiguren and J. Urrestilla, The scaling density of axion strings, 1908.03522 .

[90] C. Ringeval, M. Sakellariadou and F. Bouchet, Cosmological evolution of cosmic string loops, JCAP 0702 (2007) 023, [astro-ph/0511646].

[91] V. Vanchurin, K. D. Olum and A. Vilenkin, Scaling of cosmic string loops, Phys. Rev. D74 (2006) 063527, [gr-qc/0511159].

[92] C. J. A. P. Martins and E. P. S. Shellard, Fractal properties and small-scale structure of cosmic string networks, Phys. Rev. D73 (2006) 043515, [astro-ph/0511792].

[93] K. D. Olum and V. Vanchurin, Cosmic string loops in the expanding Universe, Phys. Rev. D75 (2007) 063521, [astro-ph/0610419].

[94] J. J. Blanco-Pillado, K. D. Olum and B. Shlaer, Large parallel cosmic string simulations: New results on loop production, Phys. Rev. D83 (2011) 083514, [1101.5173].

[95] LIGO Scientific, VIRGO collaboration, J. Aasi et al., Characterization of the LIGO detectors during their sixth science run, Class. Quant. Grav. 32 (2015) 115012, [1410.7764].

[96] K. Yagi and N. Seto, Detector configuration of DECIGO/BBO and identification of cosmological neutron-star binaries, Phys. Rev. D83 (2011) 044011, [1101.3940].

[97] LISA collaboration, H. Audley et al., Laser Interferometer Space Antenna, 1702.00786.

[98] S. Hild et al., Sensitivity Studies for Third-Generation Gravitational Wave Observatories, Class. Quant. Grav. 28 (2011) 094013, [1012.0908].

[99] M. Punturo et al., The Einstein Telescope: A third-generation gravitational wave observatory, Class. Quant. Grav. 27 (2010) 194002.

[100] LiGO Scientific collaboration, B. P. Abbott et al., Exploring the Sensitivity of Next Generation Gravitational Wave Detectors, Class. Quant. Grav. 34 (2017) 044001, [1607.08697].

[101] J. J. Blanco-Pillado, K. D. Olum and B. Shlaer, The number of cosmic string loops, Phys. Rev. D89 (2014) 023512, [1309.6637].

[102] L. M. Krauss, Gravitational waves from global phase transitions, Phys. Lett. B284 (1992) 229-233. 
[103] K. Jones-Smith, L. M. Krauss and H. Mathur, A Nearly Scale Invariant Spectrum of Gravitational Radiation from Global Phase Transitions, Phys. Rev. Lett. 100 (2008) 131302, [0712.0778].

[104] E. Fenu, D. G. Figueroa, R. Durrer and J. Garcia-Bellido, Gravitational waves from self-ordering scalar fields, JCAP 0910 (2009) 005, [0908.0425].

[105] D. G. Figueroa, M. Hindmarsh and J. Urrestilla, Exact Scale-Invariant Background of Gravitational Waves from Cosmic Defects, Phys. Rev. Lett. 110 (2013) 101302, [1212.5458].

[106] J. J. Blanco-Pillado, K. D. Olum and X. Siemens, New limits on cosmic strings from gravitational wave observation, Phys. Lett. B778 (2018) 392-396, [1709.02434].

[107] M. Maggiore, Gravitational waves: Volume 1: Theory and experiments, vol. 1. Oxford university press, 2008.

[108] T. W. B. Kibble and N. Turok, Selfintersection of Cosmic Strings, Phys. Lett. 116B (1982) 141-143.

[109] T. Damour and A. Vilenkin, Gravitational wave bursts from cusps and kinks on cosmic strings, Phys. Rev. D64 (2001) 064008, [gr-qc/0104026].

[110] C. Ringeval and T. Suyama, Stochastic gravitational waves from cosmic string loops in scaling, JCAP 1712 (2017) 027, [1709.03845].

[111] C. J. Burden, Gravitational Radiation From a Particular Class of Cosmic Strings, Phys. Lett. 164B (1985) 277-281.

[112] T. Damour and A. Vilenkin, Gravitational wave bursts from cosmic strings, Phys. Rev. Lett. 85 (2000) 3761-3764, [gr-qc/0004075].

[113] A. Vilenkin and T. Vachaspati, Radiation of Goldstone Bosons From Cosmic Strings, Phys. Rev. D35 (1987) 1138.

[114] D. Bettoni, G. Domènech and J. Rubio, Gravitational waves from global cosmic strings in quintessential inflation, JCAP 1902 (2019) 034, [1810.11117].

[115] A. Vilenkin and A. E. Everett, Cosmic Strings and Domain Walls in Models with Goldstone and PseudoGoldstone Bosons, Phys. Rev. Lett. 48 (1982) 1867-1870.

[116] A. Vilenkin, Cosmic Strings and Domain Walls, Phys. Rept. 121 (1985) 263-315.

[117] P. Sikivie, Of Axions, Domain Walls and the Early Universe, Phys. Rev. Lett. 48 (1982) 1156-1159.

[118] J. J. Blanco-Pillado and K. D. Olum, The Form of cosmic string cusps, Phys. Rev. D59 (1999) 063508, [gr-qc/9810005].

[119] L. Lentati et al., European Pulsar Timing Array Limits On An Isotropic Stochastic Gravitational-Wave Background, Mon. Not. Roy. Astron. Soc. 453 (2015) 2576-2598, [1504.03692].

[120] NANOGRAV collaboration, Z. Arzoumanian et al., The NANOGrav 11-year Data Set: Pulsar-timing Constraints On The Stochastic Gravitational-wave Background, Astrophys. J. 859 (2018) 47, [1801.02617]. 
[121] M. Sakellariadou, A Note on the evolution of cosmic string/superstring networks, JCAP 0504 (2005) 003, [hep-th/0410234].

[122] T. Damour and A. Vilenkin, Gravitational radiation from cosmic (super)strings: Bursts, stochastic background, and observational windows, Phys. Rev. D71 (2005) 063510, [hep-th/0410222].

[123] M. Breitbach, J. Kopp, E. Madge, T. Opferkuch and P. Schwaller, Dark, Cold, and Noisy: Constraining Secluded Hidden Sectors with Gravitational Waves, JCAP 1907 (2019) 007, [1811.11175].

[124] LigO Scientific, Virgo collaboration, B. P. Abbott et al., Constraints on cosmic strings using data from the first Advanced LIGO observing run, Phys. Rev. D97 (2018) 102002, [1712.01168].

[125] LigO Scientific, Virgo collaboration, B. P. Abbott et al., All-sky search for short gravitational-wave bursts in the second Advanced LIGO and Advanced Virgo run, 1905.03457.

[126] L. Lorenz, C. Ringeval and M. Sakellariadou, Cosmic string loop distribution on all length scales and at any redshift, JCAP 1010 (2010) 003, [1006.0931].

[127] G. Vincent, N. D. Antunes and M. Hindmarsh, Numerical simulations of string networks in the Abelian Higgs model, Phys. Rev. Lett. 80 (1998) 2277-2280, [hep-ph/9708427].

[128] M. Hindmarsh, S. Stuckey and N. Bevis, Abelian Higgs Cosmic Strings: Small Scale Structure and Loops, Phys. Rev. D79 (2009) 123504, [0812.1929].

[129] M. Hindmarsh, J. Lizarraga, J. Urrestilla, D. Daverio and M. Kunz, Scaling from gauge and scalar radiation in Abelian Higgs string networks, Phys. Rev. D96 (2017) 023525, [1703.06696].

[130] J. N. Moore and E. P. S. Shellard, On the evolution of Abelian Higgs string networks, hep-ph/9808336.

[131] K. D. Olum and J. J. Blanco-Pillado, Radiation from cosmic string standing waves, Phys. Rev. Lett. 84 (2000) 4288-4291, [astro-ph/9910354].

[132] J. N. Moore, E. P. S. Shellard and C. J. A. P. Martins, On the evolution of Abelian-Higgs string networks, Phys. Rev. D65 (2002) 023503, [hep-ph/0107171].

[133] J. J. Blanco-Pillado, K. D. Olum and B. Shlaer, Cosmic string loop shapes, Phys. Rev. D92 (2015) 063528, [1508.02693].

[134] D. Matsunami, L. Pogosian, A. Saurabh and T. Vachaspati, Decay of Cosmic String Loops Due to Particle Radiation, https: // ayushsaurabh. home. blog. .

[135] J. V. Rocha, Analytic Approaches to the Study of Small Scale Structure on Cosmic String Networks, 0812.4020.

[136] J. M. Quashnock and D. N. Spergel, Gravitational Selfinteractions of Cosmic Strings, Phys. Rev. D42 (1990) 2505-2520.

[137] J. J. Blanco-Pillado, K. D. Olum and J. M. Wachter, Gravitational backreaction near cosmic string kinks and cusps, Phys. Rev. D98 (2018) 123507, [1808.08254]. 
[138] J. J. Blanco-Pillado, K. D. Olum and J. M. Wachter, Gravitational backreaction simulations of simple cosmic string loops, Phys. Rev. D100 (2019) 023535, [1903.06079].

[139] J. M. Wachter and K. D. Olum, Gravitational backreaction on piecewise linear cosmic string loops, Phys. Rev. D95 (2017) 023519, [1609.01685].

[140] J. M. Wachter and K. D. Olum, Gravitational smoothing of kinks on cosmic string loops, Phys. Rev. Lett. 118 (2017) 051301, [1609.01153].

[141] K. D. Olum and J. J. Blanco-Pillado, Field theory simulation of Abelian Higgs cosmic string cusps, Phys. Rev. D60 (1999) 023503, [gr-qc/9812040].

[142] T. Elghozi, W. Nelson and M. Sakellariadou, Cusps and pseudocusps in strings with Y-junctions, Phys. Rev. D90 (2014) 123517, [1403.3225].

[143] M. J. Stott, T. Elghozi and M. Sakellariadou, Gravitational Wave Bursts from Cosmic String Cusps and Pseudocusps, Phys. Rev. D96 (2017) 023533, [1612.07599].

[144] P. Binetruy, A. Bohe, T. Hertog and D. A. Steer, Gravitational wave signatures from kink proliferation on cosmic (super-) strings, Phys. Rev. D82 (2010) 126007, [1009.2484].

[145] P. Binetruy, A. Bohe, T. Hertog and D. A. Steer, Proliferation of sharp kinks on cosmic (super-)string loops with junctions, Phys. Rev. D82 (2010) 083524, [1005.2426].

[146] P. Bhattacharjee and G. Sigl, Origin and propagation of extremely high-energy cosmic rays, Phys. Rept. 327 (2000) 109-247, [astro-ph/9811011].

[147] S. Laliberte and R. Brandenberger, Ionization from Cosmic Strings at Cosmic Dawn, 1907.08022

[148] C. J. Hogan, Gravitational Waves from Light Cosmic Strings: Backgrounds and Bursts with Large Loops, Phys. Rev. D74 (2006) 043526, [astro-ph/0605567].

[149] J. J. Blanco-Pillado and K. D. Olum, Stochastic gravitational wave background from smoothed cosmic string loops, Phys. Rev. D96 (2017) 104046, [1709.02693].

[150] P. Auclair, C. Ringeval, M. Sakellariadou and D. Steer, Cosmic string loop production functions, JCAP 1906 (2019) 015, [1903.06685].

[151] J. Polchinski and J. V. Rocha, Analytic study of small scale structure on cosmic strings, Phys. Rev. D74 (2006) 083504, [hep-ph/0606205].

[152] J. Polchinski and J. V. Rocha, Cosmic string structure at the gravitational radiation scale, Phys. Rev. D75 (2007) 123503, [gr-qc/0702055].

[153] F. Dubath, J. Polchinski and J. V. Rocha, Cosmic String Loops, Large and Small, Phys. Rev. D77 (2008) 123528, [0711.0994].

[154] J. V. Rocha, Scaling solution for small cosmic string loops, Phys. Rev. Lett. 100 (2008) 071601, [0709.3284].

[155] J. J. Blanco-Pillado, K. D. Olum and J. M. Wachter, Energy-conservation constraints on cosmic string loop production and distribution functions, 1907.09373. 
[156] LigO Scientific, Virgo collaboration, B. P. Abbott et al., GW150914: First results from the search for binary black hole coalescence with Advanced LIGO, Phys. Rev. D93 (2016) 122003, [1602.03839].

[157] LiGO Scientific, Virgo collaboration, B. P. Abbott et al., GW151226: Observation of Gravitational Waves from a 22-Solar-Mass Binary Black Hole Coalescence, Phys. Rev. Lett. 116 (2016) 241103, [1606.04855].

[158] Ligo Scientific, Virgo collaboration, B. P. Abbott et al., Binary Black Hole Mergers in the first Advanced LIGO Observing Run, Phys. Rev. X6 (2016) 041015, [1606.04856].

[159] LiGO Scientific, VIRGO collaboration, B. P. Abbott et al., GW170104: Observation of a 50-Solar-Mass Binary Black Hole Coalescence at Redshift 0.2, Phys. Rev. Lett. 118 (2017) 221101, [1706.01812].

[160] LigO Scientific, Virgo collaboration, B. P. Abbott et al., GW170608: Observation of a 19-solar-mass Binary Black Hole Coalescence, Astrophys. J. 851 (2017) L35, [1711.05578].

[161] LigO Scientific, Virgo collaboration, B. P. Abbott et al., GW170814: A Three-Detector Observation of Gravitational Waves from a Binary Black Hole Coalescence, Phys. Rev. Lett. 119 (2017) 141101, [1709.09660].

[162] LigO Scientific, Virgo collaboration, B. P. Abbott et al., GWTC-1: A Gravitational-Wave Transient Catalog of Compact Binary Mergers Observed by LIGO and Virgo during the First and Second Observing Runs, 1811.12907.

[163] LigO Scientific, Virgo collaboration, B. P. Abbott et al., GW170817: Observation of Gravitational Waves from a Binary Neutron Star Inspiral, Phys. Rev. Lett. 119 (2017) 161101, [1710.05832].

[164] T. Venumadhav, B. Zackay, J. Roulet, L. Dai and M. Zaldarriaga, New Binary Black Hole Mergers in the Second Observing Run of Advanced LIGO and Advanced Virgo, 1904.07214.

[165] LigO Scientific, Virgo collaboration, B. P. Abbott et al., GW170817: Implications for the Stochastic Gravitational-Wave Background from Compact Binary Coalescences, Phys. Rev. Lett. 120 (2018) 091101, [1710.05837].

[166] C. Cutler and J. Harms, BBO and the neutron-star-binary subtraction problem, Phys. Rev. D73 (2006) 042001, [gr-qc/0511092].

[167] T. Regimbau, M. Evans, N. Christensen, E. Katsavounidis, B. Sathyaprakash and S. Vitale, Digging deeper: Observing primordial gravitational waves below the binary black hole produced stochastic background, Phys. Rev. Lett. 118 (2017) 151105, [1611.08943].

[168] A. J. Farmer and E. S. Phinney, The gravitational wave background from cosmological compact binaries, Mon. Not. Roy. Astron. Soc. 346 (2003) 1197, [astro-ph/0304393].

[169] P. A. Rosado, Gravitational wave background from binary systems, Phys. Rev. D84 (2011) 084004, [1106.5795].

[170] C. J. Moore, R. H. Cole and C. P. L. Berry, Gravitational-wave sensitivity curves, Class. Quant. Grav. 32 (2015) 015014, [1408.0740]. 
[171] D. I. Kosenko and K. A. Postnov, On the gravitational wave noise from unresolved extragalactic binaries, Astron. Astrophys. 336 (1998) 786, [astro-ph/9801032].

[172] M. R. Adams and N. J. Cornish, Discriminating between a Stochastic Gravitational Wave Background and Instrument Noise, Phys. Rev. D82 (2010) 022002, [1002.1291].

[173] M. R. Adams and N. J. Cornish, Detecting a Stochastic Gravitational Wave Background in the presence of a Galactic Foreground and Instrument Noise, Phys. Rev. D89 (2014) 022001, [1307.4116].

[174] X.-J. Zhu, E. J. Howell, D. G. Blair and Z.-H. Zhu, On the gravitational wave background from compact binary coalescences in the band of ground-based interferometers, Mon. Not. Roy. Astron. Soc. 431 (2013) 882-899, [1209.0595].

[175] C. J. A. P. Martins and E. P. S. Shellard, Extending the velocity dependent one scale string evolution model, Phys. Rev. D65 (2002) 043514, [hep-ph/0003298].

[176] J. R. C. C. C. Correia and J. A. P. Martins, Extending and Calibrating the Velocity dependent One-Scale model for Cosmic Strings with One Thousand Field Theory Simulations, Phys. Rev. D100 (2019) 103517, [1911.03163].

[177] C. J. A. P. Martins and E. P. S. Shellard, String evolution with friction, Phys. Rev. D53 (1996) 575-579, [hep-ph/9507335].

[178] C. J. A. P. Martins and E. P. S. Shellard, Quantitative string evolution, Phys. Rev. D54 (1996) 2535-2556, [hep-ph/9602271].

[179] C. J. Martins, Defect evolution in cosmology and condensed matter: quantitative analysis with the velocity-dependent one-scale model. Springer, 2016.

[180] Particle Data Group collaboration, M. Tanabashi et al., Review of Particle Physics, Phys. Rev. D98 (2018) 030001.

[181] A. Vilenkin, Cosmic string dynamics with friction, Phys. Rev. D43 (1991) 1060-1062.

[182] Q. Shafi and A. Vilenkin, Spontaneously Broken Global Symmetries and Cosmology, Phys. Rev. D29 (1984) 1870.

[183] E. T. Vishniac, K. A. Olive and D. Seckel, Cosmic Strings and Inflation, Nucl. Phys. B289 (1987) 717-734.

[184] L. A. Kofman and A. D. Linde, Generation of Density Perturbations in the Inflationary Cosmology, Nucl. Phys. B282 (1987) 555.

[185] J. Yokoyama, Natural Way Out of the Conflict Between Cosmic Strings and Inflation, Phys. Lett. B212 (1988) 273-276.

[186] J. Yokoyama, INFLATION CAN SAVE COSMIC STRINGS, Phys. Rev. Lett. 63 (1989) 712 .

[187] M. Nagasawa and J. Yokoyama, Phase transitions triggered by quantum fluctuations in the inflationary universe, Nucl. Phys. B370 (1992) 472-490.

[188] R. Basu and A. Vilenkin, Evolution of topological defects during inflation, Phys. Rev. D50 (1994) 7150-7153, [gr-qc/9402040]. 
[189] K. Freese, T. Gherghetta and H. Umeda, Moduli inflation with large scale structure produced by topological defects, Phys. Rev. D54 (1996) 6083-6087, [hep-ph/9512211].

[190] M. Cirelli, Y. Gouttenoire, K. Petraki and F. Sala, Homeopathic Dark Matter, or how diluted heavy substances produce high energy cosmic rays, 1811.03608.

[191] C. Caprini et al., Detecting gravitational waves from cosmological phase transitions with LISA: an update, 1910.13125.

[192] C. Grojean and G. Servant, Gravitational Waves from Phase Transitions at the Electroweak Scale and Beyond, Phys. Rev. D75 (2007) 043507, [hep-ph/0607107].

[193] C. Caprini, D. G. Figueroa, R. Flauger, G. Nardini, M. Peloso, M. Pieroni et al., Reconstructing the spectral shape of a stochastic gravitational wave background with LISA, 1906.09244.

[194] A. Vilenkin, Gravitational Field of Vacuum Domain Walls and Strings, Phys. Rev. D23 (1981) 852-857.

[195] J. L. Christiansen, E. Albin, K. A. James, J. Goldman, D. Maruyama and G. F. Smoot, Search for Cosmic Strings in the GOODS Survey, Phys. Rev. D77 (2008) 123509, [0803.0027].

[196] J. L. Christiansen, E. Albin, T. Fletcher, J. Goldman, I. P. W. Teng, M. Foley et al., Search for Cosmic Strings in the COSMOS Survey, Phys. Rev. D83 (2011) 122004, [1008.0426].

[197] K. J. Mack, D. H. Wesley and L. J. King, Observing cosmic string loops with gravitational lensing surveys, Phys. Rev. D76 (2007) 123515, [astro-ph/0702648].

[198] J. R. Gott, III, Gravitational lensing effects of vacuum strings: Exact solutions, Astrophys. J. 288 (1985) 422-427.

[199] N. Kaiser and A. Stebbins, Microwave Anisotropy Due to Cosmic Strings, Nature 310 (1984) 391-393.

[200] F. R. Bouchet, D. P. Bennett and A. Stebbins, Microwave Anisotropy Patterns from Evolving String Networks, Nature 335 (1988) 410-414.

[201] J. Silk and A. Vilenkin, COSMIC STRINGS AND GALAXY FORMATION, Phys. Rev. Lett. 53 (1984) 1700-1703.

[202] L. Pogosian and T. Vachaspati, Cosmic microwave background anisotropy from wiggly strings, Phys. Rev. D60 (1999) 083504, [astro-ph/9903361].

[203] J. Lizarraga, J. Urrestilla, D. Daverio, M. Hindmarsh, M. Kunz and A. R. Liddle, Constraining topological defects with temperature and polarization anisotropies, Phys. Rev. D90 (2014) 103504, [1408.4126].

[204] J. Lizarraga, J. Urrestilla, D. Daverio, M. Hindmarsh and M. Kunz, New CMB constraints for Abelian Higgs cosmic strings, JCAP 1610 (2016) 042, [1609.03386].

[205] R. Battye and A. Moss, Updated constraints on the cosmic string tension, Phys. Rev. D82 (2010) 023521, [1005.0479]. 
[206] T. Charnock, A. Avgoustidis, E. J. Copeland and A. Moss, CMB constraints on cosmic strings and superstrings, Phys. Rev. D93 (2016) 123503, [1603.01275].

[207] A. Lopez-Eiguren, J. Lizarraga, M. Hindmarsh and J. Urrestilla, Cosmic Microwave Background constraints for global strings and global monopoles, JCAP 1707 (2017) 026, [1705.04154].

[208] Planck collaboration, P. A. R. Ade et al., Planck 2013 results. XXV. Searches for cosmic strings and other topological defects, Astron. Astrophys. 571 (2014) A25, [1303.5085].

[209] C. Ringeval and F. R. Bouchet, All Sky CMB Map from Cosmic Strings Integrated Sachs-Wolfe Effect, Phys. Rev. D86 (2012) 023513, [1204.5041].

[210] R. Ciuca and O. F. Hernández, Information Theoretic Bounds on Cosmic String Detection in CMB Maps with Noise, 1911.06378.

[211] R. Khatri and B. D. Wandelt, Cosmic (super)string constraints from $21 \mathrm{~cm}$ radiation, Phys. Rev. Lett. 100 (2008) 091302, [0801.4406].

[212] G. Sigl, D. N. Schramm and P. Bhattacharjee, On the origin of highest energy cosmic rays, Astropart. Phys. 2 (1994) 401-414, [astro-ph/9403039].

[213] V. Berezinsky, P. Blasi and A. Vilenkin, Ultrahigh-energy gamma-rays as signature of topological defects, Phys. Rev. D58 (1998) 103515, [astro-ph/9803271].

[214] V. Berezinsky, E. Sabancilar and A. Vilenkin, Extremely High Energy Neutrinos from Cosmic Strings, Phys. Rev. D84 (2011) 085006, [1108.2509].

[215] K. Greisen, End to the cosmic-ray spectrum?, Phys. Rev. Lett. 16 (Apr, 1966) 748-750.

[216] G. T. Zatsepin and V. A. Kuzmin, Upper limit of the spectrum of cosmic rays, Journal of Experimental and Theoretical Physics Letters (JETP Letters) 4 (1966) 78-80.

[217] R. Brandenberger, B. Cyr and A. V. Iyer, Fast Radio Bursts from the Decay of Cosmic String Cusps, 1707.02397.

[218] R. Brandenberger, B. Cyr and T. Schaeffer, On the Possible Enhancement of the Global 21-cm Signal at Reionization from the Decay of Cosmic String Cusps, JCAP 1904 (2019) 020, [1810.03219].

[219] R. Brandenberger, B. Cyr and R. Shi, Constraints on Superconducting Cosmic Strings from the Global 21-cm Signal before Reionization, JCAP 1909 (2019) 009, [1902.08282].

[220] N. Turok and P. Bhattacharjee, STRETCHING COSMIC STRINGS, Phys. Rev. D29 (1984) 1557.

[221] C. J. A. P. Martins, J. N. Moore and E. P. S. Shellard, A Unified model for vortex string network evolution, Phys. Rev. Lett. 92 (2004) 251601, [hep-ph/0310255].

[222] M. Maggiore, Gravitational wave experiments and early universe cosmology, Phys. Rept. 331 (2000) 283-367, [gr-qc/9909001].

[223] E. Thrane and J. D. Romano, Sensitivity curves for searches for gravitational-wave backgrounds, Phys. Rev. D88 (2013) 124032, [1310.5300]. 
[224] KAGRA, Ligo Scientific, VIRGO collaboration, B. P. Abbott et al., Prospects for Observing and Localizing Gravitational-Wave Transients with Advanced LIGO, Advanced Virgo and KAGRA, Living Rev. Rel. 21 (2018) 3, [1304.0670].

[225] LiGO Scientific, VIRGO collaboration, J. Abadie et al., Upper limits on a stochastic gravitational-wave background using LIGO and Virgo interferometers at 600-1000 Hz, Phys. Rev. D85 (2012) 122001, [1112.5004].

[226] C. M. F. Mingarelli, S. R. Taylor, B. S. Sathyaprakash and W. M. Farr, Understanding $\Omega_{\mathrm{gw}}(f)$ in Gravitational Wave Experiments, 1911.09745. 